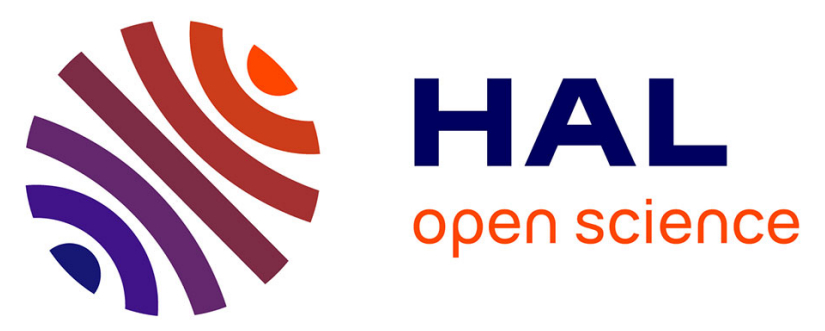

\title{
Characterization of black spot resistance in diploid roses with QTL detection, meta-analysis and candidate-gene identification
}

\author{
D. Lopez Arias, A. Chastellier, T. Thouroude, J. Bradeen, L. van Eck, \\ Yannick de Oliveira, S. Paillard, F. Foucher, L. Hibrand-Saint Oyant, Vanessa \\ Soufflet-Freslon
}

\section{To cite this version:}

D. Lopez Arias, A. Chastellier, T. Thouroude, J. Bradeen, L. van Eck, et al.. Characterization of black spot resistance in diploid roses with QTL detection, meta-analysis and candidate-gene identification. TAG Theoretical and Applied Genetics, 2020, early access, 23 p. 10.1007/s00122-020-03670-5 . hal02933267

\section{HAL Id: hal-02933267 \\ https://univ-angers.hal.science/hal-02933267}

Submitted on 8 Sep 2020

HAL is a multi-disciplinary open access archive for the deposit and dissemination of scientific research documents, whether they are published or not. The documents may come from teaching and research institutions in France or abroad, or from public or private research centers.
L'archive ouverte pluridisciplinaire HAL, est destinée au dépôt et à la diffusion de documents scientifiques de niveau recherche, publiés ou non, émanant des établissements d'enseignement et de recherche français ou étrangers, des laboratoires publics ou privés. 


\title{
CHARACTERIZATION OF BLACK SPOT RESISTANCE IN
} DIPLOID ROSES WITH QTL DETECTION, META-ANALYSIS AND CANDIDATE-GENE IDENTIFICATION

\author{
D. C. Lopez Arias ${ }^{1}$, A. Chastellier ${ }^{1}$, T. Thouroude ${ }^{1}$, J. Bradeen ${ }^{2}$, L. Van Ech , Yannick De Oliveira $^{3}$, S. \\ Paillard $^{1}$, F. Foucher ${ }^{1}$, L. Hibrand-Saint Oyant ${ }^{1}$ and V. Soufflet-Freslon ${ }^{1}$ \\ ${ }^{2}$ Department of Plant Pathology and The Stakman-Borlaug Center for Sustainable Plant Health, University of \\ Minnesota, St. Paul, MN, USA \\ ${ }^{1}$ IRHS-UMR1345, Université d'Angers, INRAE, Institut Agro, SFR 4207 QuaSaV, 49071, Beaucouzé, \\ ${ }^{3}$ Génétique Quantitative et Évolution - Le Moulon, INRAE - Université Paris-Sud - CNRS - AgroParisTech, \\ Ferme du Moulon, F-91190 Gif-sur-Yvette, France \\ *Corresponding author: Diana Carolina Lopez Arias, diana.lopez-arias@inrae.fr
}

(1)

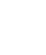

France

(4)

\section{Email list of the authors:}

D. C. Lopez Arias ${ }^{1}$ : diana.lopez-arias@inrae.fr, A. Chastellier ${ }^{1}$ : annie.chastelier@inrae.fr, T. Thouroude ${ }^{1}$ : tatiana.thouroude@inrae.fr, J. Bradeen²: jbradeen@umn.edu, L. Van Eck²: vaneck@augsburg.edu, Yannick De Oliveira $^{3}$ : yannick.de-oliveira@inrae.fr. S. Paillard ${ }^{1}$ : sophie.paillard@inrae.fr, F. Foucher ${ }^{1}$ : fabrice.foucher@inrae.fr, L. Hibrand-Saint Oyant ${ }^{1}$ : laurence.hibrand-saint-oyant@inrae.fr and V. Soufflet-Freslon ${ }^{1}$ : vanessa.souffletfreslon@Agrocampus-ouest.fr.

3

4

\author{
Author's ORCID: \\ D. C. Lopez Arias: https://orcid.org/0000-0001-8129-2786, J. Bradeen: https://orcid.org/0000-0003-1930-
} 4728, L. Van Ech: https://orcid.org/0000-0002-3073-1344, S. Paillard: https://orcid.org/0000-0001-7516-848X, F. 

V. Soufflet-Freslon: https://orcid.org/0000-0002-1264-7595.

\section{Key message}

Two environmentally stable QTLs linked to black spot disease resistance in the Rosa wichurana genetic background were detected, in different connected populations, on linkage groups 3 and 5. Co-localization between $R$ genes and defense response genes was revealed via meta-analysis.

is efficiently controlled with fungicides. However, in the actual context of reducing agrochemical use, the demand for rose bushes with higher levels of resistance has increased. Qualitative resistance conferred by major genes ( $R d r$ genes) has been widely studied but quantitative resistance to BSD requires further investigation. In this study, segregating populations connected through the BSD resistant Rosa wichurana male parent were phenotyped for disease resistance over several years and locations. A pseudo-testcross approach was used, resulting in six parental maps across three populations. A total of 45 individual QTLs with significant effect on BSD resistance were mapped on the male maps (on linkage groups (LG) B3, B4, B5 and B6), and 12 on the female maps (on LG A1, A2, A3, A4 and A5). Two major regions linked to BSD resistance were identified on LG B3 and B5 of the male maps and were integrated into a consensus map built from all three of the male maps. A meta-analysis was used to narrow down the confidence intervals of individual QTLs from three populations by generating meta-QTLs. Two "hot spots" or meta-QTLs were found per LG, enabling reduction of the confidence interval to $10.42 \mathrm{cM}$ for B3 and $11.47 \mathrm{cM}$ for B5. An expert annotation of NBS-LRR encoding genes of the genome assembly of Hibrand et al. was performed and used to explore potential colocalization with $R$-genes. Co-localization with defense response genes was also investigated.

\section{Keywords}




\section{Conflict of interest}

54 All authors declare that they have no conflicts of interests.

\section{$55 \quad$ Ethical standards}

56 We declare that these experiments complied with the ethical standards in France.

57

\section{Availability of data and material}

Genetic maps and QTL tables are available as supplementary data.

\section{Author contribution}

DCLA participated in the phenotyping, performed the genetic and statistical analyses and wrote the paper. VSF, FF and LHSO conceived the AC provided a technical support in the SSR genotyping and participated in the phenotyping. TT coordinated the field trials and participated in the phenotyping. LVE and JB performed the manual annotation and provided revision of the manuscript. YDO provided support in software Biomercator and the metaanalysis approach. FF provided extensive revisions of the manuscript. SP, LHSO and VSF participated in the phenotyping and provided extensive revisions of the manuscript. All authors read the final version of the manuscript and approved its publication.

\section{Introduction}

Cultivated roses are among the most popular garden plants (Waliczek et al. 2015a; Wang et al. 2017). In France, $16 \%$ of shrubs bought by the final consumers are garden roses, with a market value of 50.6 million Euros in 2017. Due in part to susceptibility to BSD, sales of garden roses in France declined 10\% in 2017 compared with 2016 (VAL'HOR and FranceAgriMer 2017). As such, BSD causes large economic losses and is, therefore, considered the most serious disease of cultivated roses grown outdoors.

BSD in roses, caused by the hemibiotrophic fungus Diplocarpon rosae Wolf, is globally distributed and affects nearly all modern varieties. To date, 13 races have been reported (Carlson-Nilsson 2001; Gachomo et al. 2006; Whitaker et al. 2007, 2010b; Zlesak et al. 2010; Zurn et al. 2018). Typical symptoms of this disease include dark rounded spots with fringed margins on the adaxial side of the leaves followed by chlorosis around the lesion and 
premature defoliation (Blechert and Debener 2005; Gachomo et al. 2006; Horst and Cloyd 2007). Reduced vigor in susceptible varieties can be observed, sometimes leading to death due to increased susceptibility to other stresses, like frost (Smith et al. 1989; Black et al. 1994). It is, then, important to study the rose-Diplocarpon rosae pathosystem.

The BSD fungus is obligate to the genus Rosa, though other Diplocarpon species are pathogenic on different crop species in the Rosaceae genus (Horst and Cloyd 2007). Diplocarpon rosae is spread mainly through asexual spores that are water-borne. During the infection process, the spores germinate, develop a germ tube with a melanized appresorium and penetrate the leaf cuticle (Gachomo 2005; Gachomo et al. 2006, 2010; Gachomo and Kotchoni 2007). During the biotrophic phase, subcuticular hyphae are formed followed by intercellular hyphae. Haustoria can be observed in this first stage of infection (Gachomo 2005; Gachomo and Kotchoni 2007). Then, the pathogen switches from a biotrophic lifestyle to a necrotrophic one and forms necrotrophic intracellular hyphae, followed in short order by a production of acervuli and second-generation conidia. On susceptible plants, a new generation of conidia is produced within nine days after inoculation (Gachomo 2005; Gachomo et al. 2006; Gachomo and Kotchoni 2007). Conidia are then spread by water-splash to start a whole new infection process on other leaves. Effective infection requires free water and humid conditions (Saunders 1966; Wiggers et al. 1997). Consequently, BSD is especially problematic in areas with high annual precipitation and humidity (Debener 2017).

BSD is rarely observed in greenhouse production as humidity can be carefully regulated. Garden rose multiplication and grafting are mainly done in the field, and rose bushes are generally used in gardens and landscaping for aesthetic value and low maintenance requirements. Due to the perennial habit of garden roses and D. rosae overwintering in fallen leaves, new infections can appear each year if the disease is not well managed (Münnekhoff et al. 2017) and can impact final consumers such as private gardeners and public garden managers. BSD is traditionally managed with fungicide applications. To reduce risk of chemical exposure, added costs and labor associated with this type of management, today's consumers are increasingly asking for natural resistance in rose plants (Harp et al. 2009; Zlesak et al. 2010, 2017; Waliczek et al. 2015b; Byrne et al. 2019). Moreover, European countries have adopted new laws to preserve the environment that aim to decrease agrochemical use. For example in France, measures such as the Ecophyto Plan (Labbé 2014) have forbidden the use of chemicals in public landscaping and private gardens since January 2017 and 2019, respectively (Labbé 2014). These concerns have encouraged breeders and researchers to study BSD and to develop varieties with sustainable and higher levels of resistance. 
Indeed, genetic resistance is a critical challenge to successful disease management in an environmentally friendly and cost-effective manner. Researchers have studied the genetic resistance to BSD, helping to identify modern rose varieties and wild species with high levels of resistance (Wiggers et al. 1997; Carlson-Nilsson 2001; Boontiang 2003; Blechert and Debener 2005; Uggla and Carlson-Nilsson 2005; Harp et al. 2009; Schulz et al. 2009; SouffletFreslon et al. 2019). However, breeding for BSD resistance is complex due to the development of new pathogenic races and different ploidy levels in the genus Rosa (Debener et al. 1998; Uggla and Carlson-Nilsson 2005; Whitaker et al. 2007; Debener 2017). Four major loci associated with BSD resistance were identified mostly using detached leaf assays: $R d r 1$ and $R d r 2$ on chromosome 1 (Malek and Debener 1998; Yokoya et al. 2000; Malek et al. 2000; Kaufmann et al. 2003, 2010; Hattendorf et al. 2004; Whitaker et al. 2010a; Terefe-Ayana et al. 2011; Menz et al. 2018, 2020), $R d r 3$ recently found to be located on chromosome 6 (Whitaker et al. 2010a; Zurn et al. 2020) and $R d r 4$ possibly located on chromosome 5 (Zurn et al. 2018). Evidence of partial resistance to BSD was reported in several cases and seems to be race-non-specific (Xue and Davidson 1998; Shupert 2006; Whitaker and Hokanson 2009; Whitaker et al. 2010b; Allum et al. 2010; Dong et al. 2017). Quantitative resistance often confers a partial level of resistance to the plant by reducing pathogen multiplication, plant colonization, disease spread or symptom severity (Pilet-Nayel et al. 2017). This type of resistance is usually controlled by several genes and is associated with genomic regions termed Quantitative Trait Loci (QTLs). Over the last 20 years, many QTL experiments for disease resistance have been carried out on major crops (Lacape et al. 2010; Qi et al. 2011; Holland and Coles 2011; Yadava et al. 2012; Hamon et al. 2013; Semagn et al. 2013; Said et al. 2013; Pilet-Nayel et al. 2017). More recently, QTL analyses have been conducted on ornamental crops such as carnations, gerberas or roses (Yagi et al. 2006, 2012; Leus et al. 2015; Fu et al. 2017; Yan et al. 2019). On-going research suggests QTLs conferring resistance to BSD. Yan et al. (Yan et al. 2019) have identified a possible major QTL on LG 3, based on one year of phenotypic data and a pedigree based map of 15 diploid genotypes while Soufflet-Freslon et al. (Soufflet-Freslon et al. 2019) identified possible QTLs in two populations in France. These emerging studies aside, quantitative resistance to BSD has not been extensively studied, yet it remains a powerful tool to identify genomic regions involved in complex traits such as disease resistance.

The validity of QTL mapping results is influenced by many factors, including experimental conditions, choice of parents, rating scale, environmental conditions during the scored years, type and size of mapping populations, map density and statistical methods (Lanaud et al. 2009; Liu et al. 2009; Vasconcellos et al. 2017; Guo et al. 2018). Thus, QTL mapping of one trait across multiple populations and environments can frequently yield heterogeneous results 
comprising different genomic positions and large confidence intervals. Further refinement of these intervals and synthesis of all QTL information are difficult but necessary steps prior to QTL cloning attempts and MAS breeding application. Meta-analysis is an effective approach to combine QTL results from independent studies and refine QTL positions on a consensus genetic map (Goffinet and Gerber 2000; Veyrieras et al. 2007). This method was useful in characterizing the genetic determinants of complex traits for a variety of crops such as drought stress in rice (Khowaja et al. 2009), ear emergence in wheat (Griffiths et al. 2009), yield and anthesis silking in maize (Semagn et al. 2013), yield associated traits in Brassica juncea (Yadava et al. 2012), seed weight and resistance to soybean cyst nematode in soybean (Guo et al. 2006; Qi et al. 2011), partial resistance to Aphanomyces euteiches in pea (Hamon et al. 2013) or fiber quality, yield, drought tolerance and disease resistance in cotton (Lacape et al. 2010; Said et al. 2013). With plant genome sequences becoming increasingly available, it is now possible to apply comparative analysis between genetic and physical maps to identify candidate genes underlying MetaQTLs. This approach has not yet been explored for an ornamental crop such as roses.

The main objective of our study was to explore the genetic determinants of BSD resistance in Rosa wichurana published rose genome assembly from Hibrand-Saint Oyant et al. in 2018.

\section{Material and methods} susceptible to BSD, and RW (Lopez Arias et al. in press; Hibrand-Saint Oyant et al. 2018; Soufflet-Freslon et al. 2019), et al. 1994), susceptible to BSD, and RW (Soufflet-Freslon et al. 2019), 
160 (Kawamura et al. 2015). In the Earth-Kind® Trials using detached leaf assay, TF was shown to be susceptible to 161 pathogen races 3, 8 and 9 (Zlesak et al. 2010).

Facility (Beaucouzé, France). The HW population was grafted on to Rosa canina 'Laxa' rootstock and planted in three

164 French sites (Soufflet-Freslon et al. 2019): powdery mildew development, and an aphicide spray was used if necessary. NIMROD® is not effective against $D$. rosae and no broad-spectrum fungicide was used. with three replicates. Due to production problems, only one replicate per individual for the OW population was used. rating scale from 0 to 5 presented in Supplementary figure 1 (Marolleau et al. 2020; Soufflet-Freslon et al. 2019). These scores correspond to a percentage of infected leaves and a degree of defoliation (scores 4 and 5). Disease resistance or susceptibility on field trials was assessed using the visual rating scale described above. First, plants with a score lower than 1 were considered "resistant". Indeed, the response to the $D$. rosae infection seemed to prevent pathogen development on the leaves or considerably reduce pathogen growth on the plant, leading to no visible symptoms (score 0 ) or to the infection of a small portion of leaves (less than $25 \%$ of the lower part of the plant). Then, plants with a score lower than 4 were considered "intermediate" as less than $75 \%$ of the lower part of the plant was infected but no defoliation had yet occurred. Finally, susceptible plants were scored 4 to 5 with more than $75 \%$ of the 
lower part of the plant showing symptoms and/or a sever defoliation at that time, meaning that the response to the infection did not efficiently impair pathogen development.

The OW population was scored from 2014 to 2018 (Lopez Arias et al. in press), the HW population was scored from 2012 to 2014 (Soufflet-Freslon et al. 2019) and in 2018, and the FW population was scored in 2014 and 2018.

All phenotypic data were analyzed with Rstudio interface (version 1.1.463, http://www.rstudio.com/) of R software (http://www.r-project.org/), version 3.5.1 (2018-07-02).

An overall mean for each population was calculated for each year of scoring to assess whether or not the disease incidence was uniform over the years using a Kruskal-Wallis test (for OW and HW populations) and a Wilcoxon test (for FW population).

The normality of residuals was tested using a Shapiro-Wilk test and the homogeneity of variance was assessed with a Bartlett's test. However, in field situations, plants can die or disease scoring can be missing, resulting in unbalanced experimental designs. Unlike analysis of variance (ANOVA), maximum likelihood methods, such as Restricted Maximum Likelihood (REML), provide unbiased estimators without any demand on the design and balance of data (Patterson and Thompson 1971; Corbeil and Searle 1976; Harville 1977; Lynch and Walsh 1998; Holland et al. 2010). Based on the data distribution, REML was used in place of ANOVA to estimate variance components using the package 'sommer' (Covarrubias-Pazaran 2019) in R. The broad-sense heritability was calculated for each population based on the following formula:

$$
H^{2}=\sigma_{g}^{2} /\left(\sigma_{g}^{2}+\frac{\sigma_{g x e}^{2}}{a}+\frac{\sigma_{\varepsilon}^{2}}{a r}\right) \text {, where } \sigma_{g}^{2} \text { is the genotypic variance among populations, } \sigma_{g x e}^{2} \text { is the variance of }
$$
the interaction between genotype and environment (year/location for HW and year for FW and OW), $\sigma_{\varepsilon}^{2}$ is the residual variance, $a$ is the number of scoring years and $r$ the number of replicates in each population.

\section{Genetic data and linkage mapping}

It is not possible to obtain homozygous parental lines in rose by self-fertilizing due to strong inbreeding depression. As such, a "double pseudo-testcross strategy" developed for highly heterozygous plants (Grattapaglia and Sederoff 1994; Weber et al. 2003; Fischer et al. 2004; Hibrand-Saint Oyant et al. 2007; Roman et al. 2015; Zurn et al. 2018; Bourke et al. 2018; Yan et al. 2019) was used in the current study. This strategy involves a controlled cross between two genotypes, with heterozygous genetic markers originating from either parents, followed in their F1 
213 generation. Multiple maximum likelihood mapping was conducting using JoinMap V4.1(Van Ooijen 2011) using the

$214 \mathrm{CP}$ (Cross population $=$ outbreed full-sib family) population setting. In this analysis, molecular markers can segregate

215 in one or both parents, with maps comprising dominant and co-dominant markers calculated separately for each parent

216 followed by construction of an integrated consensus map.

Parental linkage maps for FW and HW populations were constructed from previously published data (Kawamura et al. 2015; Roman et al. 2015). Constructed maps were checked for apparent genotyping errors and/or poor fitting loci by looking at aberrant number of double crossing, high values of 'Nearest Neighbor fit' (N.N fit) and genotype probabilities (-Log10(P)). When outstanding loci were found, genotyping was controlled either with $\mathrm{SNP} /$ probeset categories according to cluster properties or raw genotyping from GeneMapper software and removed when necessary. Maps were then re-calculated. The consensus map released by (Spiller et al. 2011) was used to check LG names and whole LG inversions for consistency.

Parental linkage maps for the OW population were developed using previously reported single nucleotide polymorphism (SNP) marker data (Hibrand-Saint Oyant et al. 2018). Short sequence repeat (SSR) markers previously genotyped in this population were also included (Lopez Arias et al. in press) and used as common markers for the Meta-QTL analysis. Parental maps were calculated using JoinMap 4.1 (Van Ooijen 2011) but due to insufficient "anchor loci" (i.e. makers heterozygous in both parents) an integrated map could not be obtained for this population. Following calculation of pair-wise recombination frequencies, seven LGs were identified using the logarithm of odds (LOD) score of independence between pairs of loci at a threshold of 4. Due to a large number of markers per LG (from 171 to 1,134 markers), the maximum-likelihood mapping algorithm method was used to order markers on LGs. Indeed, this method is more suitable than regression mapping for large marker datasets (Cheema and Dicks 2009). The maximum-likelihood mapping algorithm method was carried out under default JoinMap calculation settings (i.e. simulated annealing chain length of 10,000 with an acceptance probability threshold of 0.25 , Gibbs sampling for estimation of multipoint recombination frequencies with a burn-in chain length of 10,000 and a chain length per Monte Carlo EM algorithm of 1,000). The same procedure previously described was carried out to identify and remove markers with genotyping errors and poor fitting. 

different years were analyzed separately. The R/QTL package (Broman and Sen 2009; Broman and Wu 2019) was used to map QTLs and specific analyses were carried out for each set of phenotypic data according to the distribution of disease scores. For all populations, scoring years and locations, a “one dimensional QTL scan” was carried out using different specific models that best fit the data using the package R/QTL on the Rstudio interface (Broman and Sen 2009).

For data distributed normally, a three-step strategy was employed using a normal model for simple interval mapping (SIM) and composite interval mapping (CIM). First, the multiple imputation method was used to overcome issues with missing data as it fills in all missing genotype data even at sites between markers on a defined grid along LGs (Broman and Sen 2009). For this, the function 'sim.geno' was used with 200 imputations and a step size of 1cM. Next, using the previous simulated data, a genome scan with a single QTL model (SIM) was performed with the function 'scanone' (parameters: normal model and imputation method). Finally, a permutation test with 1,000 permutations of the data was used to identify genome-wide significance thresholds for declaring the presence of a QTL (Doerge and Churchill 1996). When QTLs were identified, CIM was performed to improve further QTL detections by including a marker at the peak of QTL with large effect as covariate (Broman and Sen 2009).

For data exhibiting a substantial peak at zero, the mapping strategy was based on a two-part model used to better characterize the influence of a QTL presence on BSD (Broman 2003; Broman and Sen 2009; Holland and Coles 2011). For this approach, the phenotypic data were analyzed in two parts. First, individuals with scoring values above zero were analyzed using a normal phenotype model. Second, the trait was considered as binary $(0$ and $>0)($ Broman 2003; Broman and Sen 2009). The two-part model performs the following three calculations, each with a resulting LOD score: (1) the hypothesis that a given QTL increased the probability that an individual had a null phenotype (LOD $\pi$ ), and so was resistant (which can also be referred as the penetrance of the disease); (2) the hypothesis that a detected QTL influenced the average phenotype among individuals with non-null phenotypes (LOD $\mu$ ), so it affected the severity of the disease; (3) the probability of having a QTL at a given position (LOD $\mu, \pi)$ (simply the sum of LOD scores from the preceding analyses). 


\section{Meta-QTL analysis}

The meta-QTL analysis was conducted with Biomercator v4.2.2 software (Arcade et al. 2004; Sosnowski et al. 2012). The total number of QTLs per LG for all three populations was recorded. LGs with more than three QTLs and with QTLs from all the populations were considered for the meta-analysis. Input map files and QTL files for Biomercator v4.2.2 were prepared for the male parent map of each population according to its requirements.

Using the male map of OW population as the reference linkage map to fix locus order, a consensus male map was constructed using ConsMap (based on a weighted least square (WLS) strategy). To identify meta-QTLs, we pursued the approach proposed by Goffinet and Gerber in 2000. Due to the fact that our mapping populations share a common male parent and because the same plants were phenotyped across years, our data are not completely independent. In simulation tests, the Goffinet approach proved to be robust, obviating the need for further manipulation of non-independent data (Goffinet and Gerber 2000). The method first tests the likelihood of QTL grouping in maximum four groups and then selects the optimum number of groups using an 'Akaike' information criterion (AIC) (Arcade et al. 2004; Sosnowski et al. 2012). Estimated positions and confidence intervals (CI) of all consensus QTLs were provided and the resulting meta-QTLs were projected on the previously constructed consensus map using QTLProj.

\section{NB-encoding genes and candidate-gene mining}

In version 4.2.2 of Biomercator, genetic maps can be linked to genome annotation using anchor markers. Genes underlying QTL or meta-QTL confidence intervals (CI) can be listed and basic gene ontology (GO) term representation within CI, chromosome or whole genome can be applied. In this study, we compared the lists of genes underlying meta-QTLs for the recently released rose genome sequence (Hibrand-Saint Oyant et al. 2018).

To detect potential candidate-genes, a detailed analysis of NBS-LRR genes was performed. Protein sequences from Rosa chinensis (Hibrand-Saint Oyant et al. 2018) were scanned for the presence of R-gene-related domains using the hmmscan algorithm in HMMER v3.1b2 (Finn et al. 2015). These domains included NB-ARC (PF00931), TIR (PF01582, PF13676) and LRR (PF00560, PF07723, PF07725, PF12799, PF13306, PF13516, PF13855, PF14580) domains as defined in the Pfam database (Finn et al. 2016). Only proteins with an NB-ARC domain hit longer than 20 amino acid residues and with E-value $<1 \times 10-05$ were retained for subsequent analysis. These sequences were 
scanned for the presence of additional, non-canonical domains. The presence of coiled-coil domains was determined for each protein using ncoils with the arguments "-c -w -win 21 -min_P 0.75" and "-c -w -win 28 -min_P 0.75" (Lupas et al. 1991). Custom python scripts were developed to parse, tile and filter the scan output to obtain detailed domain configuration for each protein sequence. NB-ARC domains were extracted from each sequence according to the hmmscan results, and used for subsequent phylogenetic analysis. NB-ARC sequences were coalesced into homology groups using USEARCH with the arguments “-cluster_agg \%s -id 0.8 -linkage min” (Edgar 2010). A multiple sequence alignment was constructed for each homology group using MUSCLE (Edgar 2004), and consensus sequences obtained using HMMBUILD and HMMEMIT in HMMER v3.1b2. A multiple sequence alignment was constructed from all consensus sequences using MUSCLE and converted to the PHYLIP format (Felsenstein 1989). RaxML was used to construct the phylogeny (Stamatakis 2014), using 100 bootstrap replicates and an NB-ARC consensus sequence from Arabidopsis thaliana as outgroup, with the arguments "-m PROTCATDAYHOFF -T 24 -f a -x 98 -p f -N 100". Chromosomal positions for candidate $R$-genes were used to construct a physical map of $R$-gene distribution within the genome, using CIRCOS (Krzywinski et al. 2009).

\section{Results}

\section{Disease scoring and statistical analyses}

BSD was recorded in all three studied populations over several environments (years and locations) under natural infection in fields with no chemical treatment. Across all scoring years, the parents RW and TF were found resistant to BSD with a mean score of 0.50 and 0.33 , respectively. The H190 female parent was classified as intermediate with a mean score of 3.44 for all locations whereas OB had a mean score of 4.7 and was classified as susceptible to BSD.

For the OW population, the mean annual BSD scores from 2014 to 2018 were not significantly different from each other $(\mathrm{H}=7.2759,4$ d.f., $\mathrm{P}=0.122$, see Supplementary figure 2$)$ indicating that the disease impact on the $\mathrm{OW}$ population was homogeneous over 2014-2015-2016-2017 and 2018. The disease scores in the OW population ranged from 0 to the maximum score 5. The median of 2018 scoring was higher than for other years. Residuals for the OW population showed a distribution close to normal $(\mathrm{W}=0.99509, \mathrm{P}=0.02045)$ but the homogeneity of variance across years was not validated by a Bartlett's test $(\mathrm{K}=298.1$, 9 d.f., $\mathrm{P}<0.001)$. 

d.f., $\mathrm{P}<0.001$, see Supplementary figure 2). In general, the FW population was more resistant than the two other populations with scores ranging from 0 to 4 . Additionally, $\mathrm{FW}$ was the only population showing no defoliation due to disease. The phenotypic distribution of the FW population showed a peak at 0 and the residuals did not follow a normal distribution ( $\mathrm{W}=0.93557, \mathrm{P}<0.001)$. However, homogeneity of variance across years was validated $(\mathrm{K}=298.1,9$ d.f., $\mathrm{P}<0.001)$.

For the HW population, the mean of BSD scores in different locations was significantly different $(\mathrm{H}=476.52$, 2 d.f., $\mathrm{P}<0.001$, data not shown), so all three locations were considered separately. In the three locations (Angers, Bellegarde and Diémoz), BSD was scored over several years (four, three and three years respectively) and infection levels for each year within a location were significantly different from each other (Ha=346.74, 3 d.f., $\mathrm{P}<0.001$; $\mathrm{Hb}=308.8$, 2 d.f., $\mathrm{P}<0.001$ and $\mathrm{Hd}=212.43$, 2 d.f., $\mathrm{P}<0.001$; respectively, see Supplementary figure 2). BSD scores ranged from 0 to 4 for Angers and Bellegarde, and no individual was found completely resistant in Diémoz in all scoring years with BSD scores ranging from 0.67 to 4. A spike-like distribution was observed in 2018 in Angers, 2014 in Bellegarde and 2013 in Diémoz. Normality of residuals as well as homogeneity of variance were not validated (respectively $\mathrm{W}=0.99618, \mathrm{P}<0.001$ and $\mathrm{K}=35.799,9$ d.f., $\mathrm{P}<0.001$ ). Interestingly, scoring year 2012 was lower than 2013 and 2014 for all three locations, and for Angers in 2018, many individuals presented a high resistance. However, the mean of BSD over the three years (2012, 2013 and 2014) was always the lowest in Angers, intermediate in Bellegarde and the highest in Diémoz.

Since normality and variance homogeneity assumptions were not validated for all three populations, the calculation of variance components was obtained using the REML method. The broad sense heritability of BSD resistance was estimated to be $0.79,0.86$ and 0.65 for the $\mathrm{OW}, \mathrm{HW}$ and FW populations, respectively, indicating that BSD resistance is mainly controlled by genetic factors in all three populations. Moreover, the proportion of phenotypic variance due to genetic effects, here the variance in response to $D$. rosae infection due to genetic background of the individuals, seems to be higher for HW than for OW and lower for FW.

\section{Linkage mapping of parental maps}

Parent-based maps were constructed for the HW and FW populations using previously published data 
to the rose integrated consensus map of (Spiller et al. 2011). For the HW population, the male map comprised 56 markers covering $418.58 \mathrm{cM}$, and the female map comprised 37 markers covering $313.89 \mathrm{cM}$. On LG6 of the female map, only two markers were found heterozygous and were mapped. For the FW population, the male map comprised 94 markers covering $457.94 \mathrm{cM}$ and the female map comprised 75 markers with a coverage of $369.91 \mathrm{cM}$.

For the OW population, we generated linkage maps with already published data by (Hibrand-Saint Oyant et al. 2018) and SSR markers selected to facilitate meta-analysis. A total of 497 SNP and 36 SSR markers were mapped on the male map. The resulting map comprises 402 unique loci assigned to seven LGs, designated B1-B7. The average marker interval was $1.14 \mathrm{cM}$ (Table 1 and Supplementary figure 4) and the entire map covered $453.62 \mathrm{cM}$. LGs range in size from 42.6 (B3) to $84.34 \mathrm{cM}$ (B2). A total of 692 SNP markers, 31 SSR markers and one gene (NP also known as RoAP2, see Table 1 and Supplementary figure 3) were mapped in the female of the OW population. The resulting map included 513 unique loci assigned to seven LGs (named A1-A7). The average distance between markers is 0.96 $\mathrm{cM}$ and LGs range in size from 33.42 (A2) to $97.65 \mathrm{cM}$ (A5). The female map for the $\mathrm{OW}$ population is $473.85 \mathrm{cM}$ in total length.

Common markers were the ones shared by at least two populations. In total, 72 markers (41 common markers found for the male maps and 31 for the female maps) were used as "bridge markers" to connect the OW, FW and HW linkage maps (Table 1, listed on Supplementary table 1). The number of common markers per LG ranged from three (B1-B6 and A1-A6-A7) to nine (B3) (Table 1). Of 41 markers shared by at least two of the male linkage map, 21 were shared by all three male maps. Only eight markers were shared by the three female maps (Supplementary table 1). There was at least one common marker between the three populations for all LGs of the male and the female (except for A1) maps.

\section{QTL analysis}

First, QTL detections within populations were performed using the parental genetic maps and phenotypic data of BSD resistance from the OW, HW and FW populations. In each population, individuals showed variable resistance responses across environments and years, so locations and years were considered separately in QTL analysis (Supplementary figure 2).

Two methods were adopted to perform QTL detection according to data distribution. Phenotypic data for the OW population and the HW population, with the exception of Angers 2018, Bellegarde 2014 and Diémoz 2013 
phenotypic data (Figure 1) were analyzed using a normal model for simple interval mapping (SIM) and composite interval mapping (CIM). All other data exhibiting spike-like distribution were analyzed with a two-part model, henceforth referred as "2p" (Broman 2003). In addition, a mean across years was applied for each population (and for the HW population for each location separately). Then a normal method for QTL detection was used.

In total with the method for normally distributed phenotypes, 35 QTLs associated with BSD resistance were detected for the male maps, and eight QTLs were detected for the female maps. For spike-like distributions, ten QTLs were detected with the male maps and four with the female maps.

\section{Male maps}

For all three populations, across years and locations combined, a total of 45 QTLs with significant effects on BSD resistance were identified on LGs B3, B4, B5 and B6 (Table 1 and Supplementary figures 5, 6, 7 and 8). QTLs on B3 and B5 were found for all three populations, whereas QTLs on B4 and B6 were population-specific (OW and HW, respectively).

QTLs on B3 were identified for all years and locations with both detection methods. Phenotypic variance within-population explained by individual QTLs on B3 varied from 3.9\% to $23.6 \%$ and the LOD values varied from 2.73 to 15.23 (Table 2). For the $\mathrm{OW}$ population, five marker peaks were found in a region from $4 \mathrm{cM}$ to $26 \mathrm{cM}$ for the different years (Supplementary figure 5 and Table 2); for the HW population, five marker peaks were also found in a region from 3 to $19 \mathrm{cM}$ for all scoring years and locations (Supplementary figures 7 and 8 ). For spike-like distributions, 2p model provides more information on the effect of the QTLs found. Indeed, for 2018 BSD scores of FW and HW in Angers and 2014 BSD scores of HW in Bellegarde, both LOD scores calculated for the 2p model exceeded their respective LOD thresholds (respectively Supplementary figures 6B, 8A and 8B, Table 2). This means that the QTLs found on B3 affects the penetrance (appearance of the symptoms) as well as the severity of the disease. However, for HW Diémoz 2013, only the LOD $\pi$ reached its corresponding threshold meaning that the QTL found on B3 for 2013 affected only the penetrance. The 2p model gave a better precision on the QTL location on B3 from the beginning of the $\mathrm{LG}$ to $9.67 \mathrm{cM}$ (Table 2).

QTLs on B5 were found repeatedly for each scoring year and location in the HW population (Supplementary figures 7 and 8), and were year dependent in the OW population (detected for scoring years 2016, 2017 and 2018, see Supplementary figure 5). A QTL on B5 was also detected on FW for scoring year 2018. The LOD peak values varied from 3.43 to 12.14 , and the phenotypic variance ranged from $2.89 \%$ to $19.82 \%$ (Table 2). For the OW population, two 
close peaks were found for scoring years 2017, 2018 and the mean in a region from 8.71cM to 10cM. For all scoring years and locations for the HW population, four marker peaks were found in a region from $23 \mathrm{cM}$ to $41 \mathrm{cM}$ (Supplementary figures 7 and 8). Interestingly, in the OW population in 2016, the FW population in 2018, and for all years and locations for the HW population, a second peak on the distal end of B5 can also be observed (Supplementary figures $5,6 \mathrm{~B}, 7$ and 8$)$.

Two QTLs were identified on B4 in 2017 and with the mean across years for the OW population, explaining 5.1 to $7.6 \%$ of the phenotypic variance. The location of the B4 QTL was defined more precisely using BSD scores averaged across years (10cM region) than a single year scores (entire LG based on 2017 data, see table 2).

QTLs with significant but small effects on BSD resistance were mapped on B6 in most years and locations for the HW population as well as for the average BSD scores. These QTLs were population-specific and were only detected when RW was crossed with H190 (Supplementary figures 7 and 8). The LOD peaks ranged from 2.6 to 5.92 explaining 3.6 to $4.63 \%$ of phenotypic variance (Table 2). For data from HW Angers 2018 and HW Bellegarde 2014, the QTL on B6 affected the penetrance of the trait (Supplementary figure 8). No interactions between QTLs were observed across years and locations (data not shown). As B6 and B4 QTLs had small effects on BSD resistance and were population-specific and year-specific, they were not considered for the subsequent meta-analysis.

\section{Female maps}

No QTL common across all three populations was identified on the female maps (Table 3), but QTLs were shared by pairs of populations. The OW and FW populations displayed a common QTL on A1, while for the HW and FW populations displayed two common QTLs, both on A4.

In the OW population, a significant QTL on A1 was identified in 2017, explaining 6.53\% of the total phenotypic variance and was located at the end of the LG (Lopez Arias et al. in press).

For the FW population, three QTLs were detected with 2018 data using 2p method. These QTLs, located on A1, A2 and A4, explained $4.06 \%, 2.57 \%$ and $3.61 \%$ of the phenotypic variance, respectively (Table 3). Interestingly, QTLs on A1 and A4 had an effect on the penetrance of the trait (LOD 2.59 and 4.09 respectively; see Table 3) whereas the QTL on A2 had an effect on both severity and penetrance (Supplementary figure 9A).

To position the QTL detected in the OW and FW populations on LG A1, markers at the confidence interval limits and markers at the peak of the QTLs were positioned on a physical map (Supplementary figure 10). TIR-NBLRR (TNL) genes and cluster of genes from this regions identified by Menz et al. in 2020 were also mapped. Two 
clusters of $T N L$ genes, named cluster 1 (genes OB2_C to OB2_H) and cluster 2 (genes OB2_J to OB2_S), were localized near the end of chromosome 1 (supplementary figure 10). The Rdrl resistance gene from Rosa multiflora is part of cluster 2 (Malek et al. 2000; Kaufmann et al. 2003; Hattendorf et al. 2004; Terefe-Ayana et al. 2011; Menz et al. 2020). Importantly, the QTLs derived from the female parents TF and OB co-localized with both clusters of $T N L$ genes (Supplementary figure 10).

For the HW population, QTLs were identified on LG A3 in 2012 and 2014 in Angers, 2013 in Bellegarde, 2014 in Diémoz and the averages across years for each location explaining 4.4\% to 5.8\% of the phenotypic variation (Table 3). One QTL explaining 5.91\% of phenotypic variation was also identified on A3 in Bellegarde in 2014 using the 2p model. This QTL had an effect on the penetrance of the trait (Supplementary figure 9B). Also in Bellegarde, a QTL explaining 5.39\% of the phenotypic variance was identified on A5 in 2012. In Diémoz, a QTL explaining 5.77\% of the phenotypic variance was identified on A4 in 2014 (Table 3).

For both male and female maps, further QTLs and epistasis between QTLs were investigated using a two dimensional genome scan and no epistasis was significantly detected for any scoring year with normal distribution (data not shown). The percent explained by all QTLs is, then, the sum of all the phenotypic variance explained by the individual QTLs of each scoring years (Table 3).

\section{Meta-analysis}

A meta-analysis of B3 and B5 QTLs was conducted. First, a consensus male map across all populations was constructed using 41 common markers (Table 1 and Supplementary table 1). In total, B3 comprised nine markers common across the OW, FW and HW male maps and B5 comprised eight markers common across all male maps (Table 1). LG3 and LG5 of the consensus map were represented in Figure 2. Secondly, QTLs of the original maps were projected on the chosen reference map (OW population map) by the map-projection function of Biomercator 4.2.2. In total, 20 QTLs of BSD resistance were projected on the reference LG3 and 17 QTLs on the reference LG5 (Figure 2). The meta-QTL optimum number was ensured by the AIC. Two meta-QTLs were identified on both LG3 and LG5 (represented by two different colors on the LGs of figure 2). For LG3, the first meta-QTL (named Meta_1_3) had a confidence interval (CI) of $2.72 \mathrm{cM}$ and the second meta-QTL (named Meta_2_3) had a CI of $7.70 \mathrm{cM}$ (Table 4 and Figure 2). The CI for the meta-QTL on LG5 (named Meta_1_5) was $3.95 \mathrm{cM}$ and $7.52 \mathrm{cM}$ for the second one (Meta_2_5). The average CI of the 20 individual QTLs found in B3 was $20.1 \mathrm{cM}$ whereas for both predicted meta- 
QTLs together it was $10.42 \mathrm{cM}$. For B5, the individual QTL average CI was $26.62 \mathrm{cM}$ and only $11.47 \mathrm{cM}$ for both predicted meta-QTLs (Figure 2 and Table 4). The CI were approximately twice as small with the meta-analysis, which allowed us to be more precise in the location of QTLs linked to BSD resistance.

When looking at the meta-QTL contributions, we can see that the first meta-QTL (Meta_1_5) was represented exclusively by OW population QTLs that showed large peaks at the beginning of the LG (except OWpop_RW_16B5 QTL that showed two distinct peaks separated by 30cM, see Supplementary figure 5) whereas the QTLs of HW and FW populations contributed to the second meta-QTL (Meta_2_5). Curiously, individual QTLs with a wide interval and double peaks separated by 10 to $30 \mathrm{cM}$ were detected on B5 (Supplementary figure 5 to 8 ). The configuration suggests the presence of linked QTLs, requiring additional or alternative analytical approaches (Nakamichi et al. 2001; Kao and Zeng 2010). Accordingly, we attempted a two-dimensional analytical approach for normally distributed data but failed to separate more precisely the putatively linked QTLs in this region (data not shown).

\section{Gene mining from meta-QTL intervals and $N B$-encoding genes in Rosa}

\section{genome}

All SNP markers were mapped to the Rosa chinensis reference genome of Hibrand-Saint Oyant et al. (2018) and were used to project the consensus genetic map onto the reference genome. The first meta-QTL on LG3, Meta_1_3, corresponds to an approximately $2.86 \mathrm{Mb}$ genome region encompassing 291 annotated genes. The second one, Meta_2_3, represents a $3.5 \mathrm{Mb}$ genome region comprising 450 annotated genes. On LG5, the first predicted metaQTL, Meta_1_5, represents $1.80 \mathrm{Mb}$ comprising 271 annotated genes, while the second meta-QTL, Meta_2_5, represents 6.06 Mb comprising 557 genes (Table 4).

We explored genes associated with predicted meta-QTLs, focusing specifically on potential disease resistance genes. First, we annotated the predicted $N B$-encoding (Nucleotide Binding) genes in the rose genome, identifying 493 candidate $R$-genes (Supplementary table 2). Candidate $N B$-LRR genes were mapped onto the assembled pseudochromosomes of $R$. chinensis (Figure 3). $N B$-encoding genes mapped to every chromosome of the rose genome. Only nine genes mapped onto the unassembled scaffolds ( $\mathrm{rc} 00)$. Chromosome 1 contained the largest number of $N B$ encoding genes (106). Overall, the mean number of $R$-genes per chromosome was 69 . However, $N B$-encoding genes were not homogeneously distributed across chromosomes, with clustering evident, especially on the distal end of chromosomes 1, 5 and 7 (Figure 3). 

$(R C 3 G 0136400, R C 3 G 0136500, R C 3 G 0136600, R C 3 G 0145900)$ co-localized with the first meta-QTL, Meta_1_3

492 (Table 4, Figure 3 and Supplementary table 2). The manual and the automated annotations identified nearly the same number of NBS-encoding genes in the interval of the second B3 meta-QTL (Meta_2_3) encompassing four RGAs for the automated annotation and three $N B$-coding genes (RC3G0272000, RC3G0277900, and $R C 3 G 0280800)$ for the manual annotation (see table 4). In addition to these NBS-encoding genes, we investigated co-localizations of defense response genes with the Meta-QTLs. Interestingly, a gene involved in response to fungal infection (RC3G0142400) is associated with the meta-QTL Meta_1_3. This gene encodes an EMSY-LIKE 1 protein that is known to contribute to RPP7-mediated and basal immunity against a specific strain Hiks1 of Peronospora parasitica in Arabidopsis (Tsuchiya and Eulgem 2011), possibly by regulating chromatin states. Two genes encoding for P450 cytochrome also colocalized with the first meta-QTL Meta_1_3. Cytochromes P450 monooxygenases (CYP) are known to be involved in plant defense mechanisms as they mediate secondary metabolism compounds of the xenobiotic detoxification pathway. For example, the $C Y P$ gene $C Y P 736 B$ in grapevine is involved in defense responses against Xylella fastidiosa and the wheat CYP72A is involved in the defense responses to Fusarium graminearum (Schuler and Werck-Reichhart 2003; Schuler et al. 2006). Similarly, in the Solanaceae, two cytochromes P450 participate in fungal pathogen resistance (Morant et al. 2003) and a Phytophthora infestans-induced cytochrome P450 is associated with quantitative resistance to late blight in potato (Trognitz et al. 2002). Moreover, a transcription factor from the WRKY family (RC3G0261500) upstream of the NBS-encoding gene (RC3G0261700) also co-localize with the Meta_2_3. WRKY transcription factors are known to play an important role in plant immunity and have been identified as major components of the resistance to fungi (Yang et al. 2009; Pandey and Somssich 2009; Lui et al. 2017). A pathogenesis-related (PR) thaumatin gene (RC3G0264400) was also found to co-localized with Meta_2_3. This gene codes for a PR-5 type protein (also called TLP for thaumatin-like protein) that has been reported for its antifungal activity against various filamentous fungi (Chu 512 and Ng 2003; Ho et al. 2007; Singh et al. 2013; Zhang et al. 2018).

513 For LG B5, 17 QTLs were mapped on the consensus LG and two meta-QTL clusters were identified. The same genes 514 (RC5G0059300, RC5G0061300, and RC5G0061600) were annotated as R-genes with the expert annotation and the 515 automatic one for Meta_1_5 (Table 4). However, in Meta_2_5 region, five genes were not found to be $N B$-encoding 516 genes (RC5G0227800, RC5G0228700, RC5G0231700, RC5G0232700, RC5G0245000) with the expert annotation.

517 Interestingly, Meta_2_5 is close to the region where the newly identified $R d r 4$ gene giving resistance to all races of $D$. 
rosae, except the race 12, was located (Zurn et al. 2018). The meta-QTL, Meta_2_5, did not co-localize with the possible location of Rdr4 but the marker peak of the QTL found in 2016 for OW population co-localized with it (Lopez Arias et al. in press) as well as the marker peak of the QTL found in Diémoz 2013 scoring year of HW population. Moreover, a glucan synthase like gene (RC5G0249400; GLS4 gene or also called CalS8), known to be involved in the formation of callose-containing papillae in response to pathogen attack (Enrique et al.; Dong 2005; Enns et al. 2005; Dong et al. 2008; Voigt and Somerville 2009; Ellinger et al. 2013; Voigt 2014,2016), co-localized with Meta_2_5. Finally, a cluster of ten genes coding for cytochrome P450 also co-localized with Meta_2_5.

\section{Discussion} resistance (QDR) is best studied under field conditions with phenotypic measurements collected over different locations and years (Kelly and Vallejo 2006; Niks et al. 2015; Corwin and Kliebenstein 2017). In the current study, a difference of inoculum pressure or fungal strains between locations (Soufflet-Freslon et al. 2019) could explain why, for the HW population, the disease incidence was generally lower in Angers than in Bellegarde and Diémoz for a given year (Supplementary figure 2C). On the other hand, the effects of environmental conditions such as temperature, humidity or rainfall on BSD development in fields are well documented (Saunders 1966; Knight 1975; Gachomo 2005). In particular, (Saunders 1966) showed that climatic conditions play a critical role in the accumulation and spread of D. rosae inoculum. Heavy rainfalls and temperature above $14^{\circ} \mathrm{C}$ during trigger inoculum accumulation and spread of conidia from infected leaves (Saunders 1966). During the scoring period from 2012 to 2018, average precipitation in August and September of a given year was consistently lower in Angers and Bellegarde than in Diémoz. Similarly, for all populations, variation in disease impacts between years was observed, with low rainfall in August-September correlating with lower BSD scores. Conversely, in wetter locations or years (e.g., Diémoz in 2013 and Bellegarde in 2014), more individuals presented disease symptoms with BSD score distributions skewed to the left (with a lot of individuals presenting more symptoms than normal) like Diémoz. Specifically, the year 2013 (with a spike at score 4) than Diémoz (97.25 mm) also showed a spike at score 4 and a distribution skewed to the left (Figure 1C). 
Our main objective was to identify genomic regions conditioning resistance to $D$. rosae. We generated and analyzed phenotypic BSD data gathered across seven years, three locations, and three genetic populations.

The three populations used in this study were originated from interspecific crosses. The highly heterozygous nature of Rosa species is a complicating factor when it comes to genetic mapping. The pseudo-test cross mapping approach (Grattapaglia and Sederoff 1994; Kirst et al. 2004; Adam-Blondon et al. 2016), developed for highly heterozygous species, allows development of parental maps that are then combined into an integrated map. However, (Gartner et al. 2013) showed that, although integrating homologous chromosomes that have been rearranged can produce mathematically and statistically correct maps, they do not necessarily reflect the biological reality of chromosomal rearrangements and meiotic recombination that had happened during the outbred cross. Therefore, even if the gene position is highly conserved between species, genome rearrangements may exist in interspecific crosses. This can lead to significant changes in collinearity between parental genomes (Lespinasse et al. 2000; Gartner et al. 2013). For example, recently released, independently generated rose genome assemblies suggest chromosome rearrangements (Raymond et al. 2018; Hibrand-Saint Oyant et al. 2018; Smulders et al. 2019). In the current study, we observed rearrangements between the parents $\mathrm{OB}$ and RW, especially on chromosome 3 . For these reasons, we decided to apply pseudo-test cross mapping approach without integrating the parental maps at the end. QTL analyses were then carried out using two different methods of detection on separated parental maps.

Overall, many QTLs for resistance to BSD were detected in this study. Consistent with natural variation in inoculum pressure, pathogen race, and environmental conditions encountered in field studies over several years, some QTLs identified in this study were only found in a given population, location or year. For example, BSD resistance QTLs on B6 were found specifically on the male map of the HW population across all environments (Table 2). Since all three populations share the RW male parent, identification of the B6 QTLs only in the HW population for several years and locations suggests that expression of these QTLs also depends on genetic background that is crossed with $R$. wichurana. Moreover, the marker peak CTG623 of B6 QTL was found to co-localize with the possible location of Rdr3 (Zurn et al. 2020).

Importantly, other QTLs were more consistently detected across populations, locations, and years, suggesting a more robust impact on disease resistance. First, a QTL on LG A1 was mapped in two populations (FW and OW) (Supplementary figure 10), originating from the female parents TF and OB. This QTL co-localizes with a cluster of 
NB-LRR genes, including the previously reported BSD resistance gene Rdrl (Lopez Arias et al. in press; Hattendorf et al. 2004; Terefe-Ayana et al. 2011). This observation suggests the intriguing possibility that the underlying genetic basis of the A1 QTL might be a canonical NB-LRR gene, in whole or in part. In addition, regarding the difference of BSD score between both female parents (with OB very susceptible to BSD and TF resistant to it), we can hypothesize that an allelic variation in the causal gene could originate a difference in the observed phenotype. Then, a QTL conditioning BSD resistance on LG B4 was identified for two different environments and two different populations: the OW population in Angers in 2017 (male map) and the HW population in Diémoz in 2014 (H190 female map; Table 2 and 3). Resistance conditioned by this QTL may be environment- and pathogen race-dependent. Finally, QTLs on LGs B3 and B5 were found for all populations, years and locations in the male resistant parent RW but also in the H190 female map (Table 2 and 3). At the moment, the apparent mapping of QTLs from these two parents, with a clear difference in BSD resistance, on the same linkage groups could be explained by one of these two hypothesis: (1) RW and H190 QTLs co-localized meaning that both parents shared the same resistance source and the difference observed in the phenotype could be due to an allelic variation between them or (2) the QTLs originated from both parents do not co-localize meaning that the resistance source is different. Unfortunately, the maps quality did not allow us to determine precisely if the male and female QTLs on linkage group 3 and 5 co-localized (data not shown). However, consistent expression of QTLs on linkage groups 3 and 5 across environments and genetic backgrounds suggest that they are stable enough to be useful in the genetic improvement of rose resistance to D. rosae. In the end, we can see with this study that separated maps allowed us to identify two main QTLs on B3 and B5 with large effect on the resistance to BSD as well as small effect QTLs on parents that were considered tolerant or susceptible.

\section{QTLs characterization and two-part model result interpretation}

Substantial peaks in the distribution of genotype mean values are fairly common and represent one of the principal departure from the assumption of normality required to perform QTL analysis (Lander and Botstein 1989). When working with disease resistance, individuals may show high resistance so a peak at 0 can be observed. In this case, the standard QTL detection approach behave poorly and can detect spurious QTLs in regions with low genetic information (Broman 2003; Feenstra and Skovgaard 2004). To deal with this problem, Broman proposed in 2003 a "Two-part model" that combines a binary analysis (absence $v s$ presence) and a normal analysis for individuals with non-null phenotype. The trait is divided in two components: penetrance and severity (Broman 2003; Broman and Sen 

so far been applied for reproductive barriers with male sterility in house mouse and ear tip masculinization in maize (White et al. 2011, 2012; Holland and Coles 2011). In our study, we adapted when necessary the model used to perform the QTL analyses, so data distributions with a substantial spike were analyzed with the two-part model. When both the penetrance and the severity of the trait were affected by the QTLs, it meant that these positions have an effect on the appearance of the BSD symptoms or penetrance (when treated as binomial; with no symptoms $v s$ symptoms) as well as the severity of symptoms (BSD score $>0$ ).

The 2p model provided interesting information for the B3 and B5 QTLs found on the male parent maps. For Angers 2018 data from the HW and FW populations, both the penetrance and the severity of the trait were affected by the B3 QTLs, meaning that these regions impact both appearance of BSD symptoms and symptom severity. Interestingly, for the B5 QTLs in both populations, only penetrance (absence/presence of the symptoms) was impacted.

612 For the resistant female parent TF, the A2 QTL impacts both the penetrance and the severity of the trait whereas the 613 A1 and A4 QTLs impact only penetrance. quantitative trait mapping has become the first step in the dissection of the genetic factors underlying complex and quantitative traits such as disease resistance (Zhu and Zhao 2007; Rawat 2016). That way, many studies mapped quantitative disease resistance loci of crop plants over the past decades (Lacape et al. 2010; Qi et al. 2011; Holland and Coles 2011; Yadava et al. 2012; Hamon et al. 2013; Semagn et al. 2013; Said et al. 2013; Pilet-Nayel et al. 2017). Cloning and functional validation of causal gene(s) or quantitative trait nucleotide polymorphism underlying QTLs has been accomplished in maize (Yang et al. 2017) but remains a long and difficult process. Indeed, the biological bases of QDR in the defense responses are still unknown, so several reviews proposed hypotheses of mechanisms underlying Quantitative resistance loci (QRL) (Poland et al. 2009; St Clair 2010; Roux et al. 2014; Corwin and Kliebenstein 2017).

First, one crucial step facilitating cloning and validation of causal genes is the QRL position refinement. Towards this goal, in this study, we narrow the confidence interval of the most phenotypically stable and reproducible QTLs on a common consensus map and perform a meta-analysis of the corresponding LGs, B3 and B5. Two meta- 
QTL clusters were identified for both LG B3 and B5 (Figure 2 and Table 4). This method allowed us to refine the genomic regions linked to BSD resistance and to study the influence of genetic background and environment on QRL detection (Table 4). However, combination of QTL mapping results across several studies that differ with marker density, linkage, sample size, etc. is still a challenge. Even though Goffinet and Gerber's method aims to resolve the number of QTL and their location using a model selection, questions on the real number of QTL subsist. On one hand, for LG B3, both clusters found during the meta-analysis can be either two real QTLs or an artefact due to the possible rearrangement that happened in this region. For instance, not far from the meta-QTL Meta_1_3 on B3, a large rearrangement was described between the two alleles in $\mathrm{OB}$ (corresponding to recurrent blooming locus and the rearrangement of copia-like retrotransposon, Hibrand-Saint Oyant et al. 2018). On the other hand, on LG B5, uncertainty remains on whether or not the QTLs located within the same genomic region are the same QTLs. Specially, because individual QTLs present double LOD peaks characteristic of linked QTLs. Increasing the sample size of the populations in future experiments may provide a better power to separate closely linked QTLs on LG B5. Therefore, at this moment, only hypotheses can be made. For example: both clusters being completely new QTLs different from the newly identified $R d r 4$ resistance gene (Zurn et al. 2018) or the first meta-QTL Meta_1_5 being a new QTL and the Meta_2_5 being Rdr4 locus that did not co-localized with it due to map imprecisions.

Second, elucidating the molecular mechanisms underlying QRLs can assist breeders in developing cultivars with more durable levels of resistance by "making more informed and prudent decisions" (Kelly and Vallejo 2006). Indeed, it has been shown that QRLs can co-localize with major R-genes as well as defense response genes (Poland et al. 2009; St Clair 2010; Roux et al. 2014; Corwin and Kliebenstein 2017). In this study, we used the meta-QTL physical positions to inform gene mining efforts based on whole genome sequence data (Hibrand-Saint Oyant et al. 2018), specifically searching for associations between defined genetic regions and NBS-LRR and defense response genes. However, the resistance loci originated from RW and the genomic differences between both genotypes could impact our efforts. Nevertheless, the use of the haploid OB genome sequence to identify candidate genes underlying BSD resistance meta-QTLs has promise. Genes involved in pathogen recognition, signal transduction, transcription modulation and detoxification pathways were identified in the chromosomal regions linked to BSD resistance. Interestingly, Blechert and Debener in 2005 classified the interaction between D. rosae and the wild type $R$. wichurana as type 7 meaning that the fungus germinates and penetrates the cuticle but is not capable of producing hyphal structures on the host. Indeed, cell-wall appositions were observed on one to three cells resulting in visible necrotic 
areas akin to hypersensitivity reactions (HR). Genes involved in the activation of cell death (WRKY, PR-proteins,

\section{Conclusion \\ Conclusion} ROS pathway, etc.) as well as in detoxification pathways (with cytochrome P450) seem to be good candidates.

Thus, the possibility of RGAs being responsible for the observed resistance on RW is also not to be ruled out as they are in the first line of biotic stress responses like the one observed for RW by Blechert and Debener (2005).

In this study, confidence intervals of chromosomal regions conditioning quantitative resistance to $D$. rosae were reduced thanks to the meta-analysis approach. Unfortunately, at this stage of the study, no consistent markers linked to BSD resistance have been identified as the CI regions and peaks of detected QTLs vary slightly over the years and locations. A refinement of the QTL location of stable QTLs on B3 and B5 is needed prior to marker detection for marker assisted breeding and/or causal gene identification. Using the genomic resources now available such as the rose genome and the data provided in this article such as the manually annotated RGAs, the first hypothesis on the genetic basis of QDR in Rose- $D$. rosae pathosystem were made. However, at this stage, only a large number of candidate genes were identified. Further evidence supporting their involvement in the observed resistance of RW genotype need to be provided so the potential candidate genes can be narrowed down to a number that can allow experimental validation through qPCR expression profiles and/or gene inactivation. Finally, the large number of QTL detected in this study, together with the fact that some QTL did not co-colocalize with known $R d r$ genes, suggests that the number of QTL could be even larger than what has been detected with these limited populations, offering the promise of wide spectrum of resistance sources to rose breeder and scientists.

In this study, we report the first discovery and genetic characterization of quantitative resistance to BSD. We examined the contributions of a common resistant parent over several years, locations, and genetic populations. We confirm the presence of a strong effect QTLs on LG B3 and B5 from the genotype R. wichurana, consistent with complementary on-going research (Yan et al. 2019). The LG B3 and LG B5 QTLs are stable over years and effective against a wide range of D. rosae races and across various environments (Lopez Arias et al. in press; Yan et al. 2019; Soufflet-Freslon et al. 2019). We demonstrate a method to leverage meta-analyses to reduce CI of single QTLs. This method was specifically chosen for its robustness to non-full independency and it allowed us to integrate data from three different populations of perennial rose bushes. BSD data collected over seven years and multiple locations, 
provided validation of QTLs on B3 and B5, demonstrating their stability and robustness. Genes underlying these QTLs are potential breeding targets for the development of BSD resistant rose cultivars.

\section{References}

Adam-Blondon A-F, Martinez-Zapater J-M, Kole C (2016) Genetics, genomics, and breeding of grapes. CRC Press

Allum JF, Bringloe HD, Roberts AV (2010) Interactions of four pathotypes of Diplocarpon rosae with species and hybrids of Rosa. Plant Pathol 59:516-522. https://doi.org/10.1111/j.1365-3059.2009.02222.x

Arcade A, Labourdette A, Falque M, et al (2004) BioMercator: integrating genetic maps and QTL towards discovery of candidate genes. Bioinforma Oxf Engl 20:2324-2326. https://doi.org/10.1093/bioinformatics/bth230

Black WA, Byrne DH, Pemberton HB (1994) Field study of black spot resistance in rose. HortScience 29:

Blechert O, Debener T (2005) Morphological characterization of the interaction between Diplocarpon rosae and various rose species. Plant Pathol 54:82-90. https://doi.org/10.1111/j.1365-3059.2005.01118.x

Boontiang K (2003) Breeding of black spot disease resistance of garden and florist rose. PhD Thesis, 愛媛大学

Bourke PM, Gitonga VW, Voorrips RE, et al (2018) Multi-environment QTL analysis of plant and flower morphological traits in tetraploid rose. TAG Theor Appl Genet Theor Angew Genet 131:2055-2069. https://doi.org/10.1007/s00122-018-3132-4

Broman K, Sen S (2009) A Guide to QTL Mapping with R/qtl. Springer-Verlag, New York

Broman KW (2003) Mapping quantitative trait loci in the case of a spike in the phenotype distribution. Genetics 163:1169-1175

Broman KW, Wu H (2019) Qtl: tools for analyzing qtl experiments. Version 1.44-9URL https://CRAN.Rproject.org/package $=$ qtl

Byrne DH, Pemberton HB, Holeman DJ, et al (2019) Survey of the rose community: desired rose traits and research issues. Acta Hortic 189-192. https://doi.org/10.17660/ActaHortic.2019.1232.28

Carlson-Nilsson BU (2001) Evaluation of rose species and cultivars for resistance to Marssonina rosae (Diplocarpon rosae). Acta Hortic 413-417. https://doi.org/10.17660/ActaHortic.2001.547.53

Cheema J, Dicks J (2009) Computational approaches and software tools for genetic linkage map estimation in plants. Brief Bioinform 10:595-608. https://doi.org/10.1093/bib/bbp045

Chu KT, Ng TB (2003) Isolation of a large thaumatin-like antifungal protein from seeds of the Kweilin chestnut Castanopsis chinensis. Biochem Biophys Res Commun 301:364-370. https://doi.org/10.1016/S0006291X(02)02998-4

Corbeil RR, Searle SR (1976) Restricted maximum likelihood (REML) estimation of variance components in the mixed model. Technometrics 18:31-38. https://doi.org/10.2307/1267913

Corwin JA, Kliebenstein DJ (2017) Quantitative resistance: more than just perception of a pathogen. Plant Cell tpc.00915.2016. https://doi.org/10.1105/tpc.16.00915 
Covarrubias-Pazaran G (2019) Sommer: solving mixed model equations in R. Version 4.0.1URL https://CRAN.Rproject.org/package $=$ sommer

Debener T (2017) Inheritance of characteristics. In: Reference Module in Life Sciences. Elsevier, Hannover, Germany, pp 1-7

Debener T, Drewes-Alvarez R, Rockstroh K (1998) Identification of five physiological races of blackspot, Diplocarpon rosae Wolf, on roses. Plant Breed 117:267-270. https://doi.org/10.1111/j.14390523.1998.tb01937.x

Doerge RW, Churchill GA (1996) Permutation tests for multiple loci affecting a quantitative character. Genetics 142:285-294

Dong Q, Wang X, Byrne DH, Ong K (2017) Characterization of Partial Resistance to Black Spot Disease of Rosa sp. HortScience 52:49-53. https://doi.org/10.21273/HORTSCI11349-16

Dong X (2005) Functional investigation of Arabidopsis callose synthases and the signal transduction pathway. The Ohio State University

Dong X, Hong Z, Chatterjee J, et al (2008) Expression of callose synthase genes and its connection with Nprl signaling pathway during pathogen infection. Planta 229:87-98. https://doi.org/10.1007/s00425-008-0812-3

Edgar RC (2010) Search and clustering orders of magnitude faster than BLAST. Bioinformatics 26:2460-2461. https://doi.org/10.1093/bioinformatics/btq461

Edgar RC (2004) MUSCLE: Multiple sequence alignment with high accuracy and high throughput. Nucleic Acids Res 32:1792-1797. https://doi.org/10.1093/nar/gkh340

Ellinger D, Naumann M, Falter C, et al (2013) Elevated early callose deposition results in complete penetration resistance to powdery mildew in Arabidopsis. Plant Physiol 161:1433-1444. https://doi.org/10.1104/pp.112.211011

Enns LC, Kanaoka MM, Torii KU, et al (2005) Two callose synthases, GSL1 and GSL5, play an essential and redundant role in plant and pollen development and in fertility. Plant Mol Biol 58:333-349. https://doi.org/10.1007/s11103-005-4526-7

Enrique R, Siciliano F, Favaro MA, et al Novel demonstration of RNAi in citrus reveals importance of citrus callose synthase in defence against Xanthomonas citri subsp. citri. Plant Biotechnol J 9:394-407

Feenstra B, Skovgaard IM (2004) A quantitative trait locus mixture model that avoids spurious LOD score peaks. Genetics 167:959-965. https://doi.org/10.1534/genetics.103.025437

Felsenstein J (1989) PHYLIP - Phylogeny Inference Package (Version 3.2). Cladistics 5:164-166. https://doi.org/10.1111/j.1096-0031.1989.tb00562.x

Finn RD, Clements J, Arndt W, et al (2015) HMMER web server: 2015 update. Nucleic Acids Res 43:W30-W38. https://doi.org/10.1093/nar/gkv397

Finn RD, Coggill P, Eberhardt RY, et al (2016) The Pfam protein families database: towards a more sustainable future. Nucleic Acids Res 44:D279-D285. https://doi.org/10.1093/nar/gkv1344

Fischer BM, Salakhutdinov I, Akkurt M, et al (2004) Quantitative trait locus analysis of fungal disease resistance factors on a molecular map of grapevine. Theor Appl Genet 108:501-515. https://doi.org/10.1007/s00122003-1445-3 
Fu Y, van Silfhout A, Shahin A, et al (2017) Genetic mapping and QTL analysis of Botrytis resistance in Gerbera hybrida. Mol Breed 37:. https://doi.org/10.1007/s11032-016-0617-1

Gachomo EW (2005) Studies of the life cycle of Diplocarpon rosae Wolf on roses and the effectiveness of fungicides on pathogenesis. Cuvillier Verlag

Gachomo EW, Dehne H-W, Steiner U (2006) Microscopic evidence for the hemibiotrophic nature of Diplocarpon rosae, cause of black spot disease of rose. Physiol Mol Plant Pathol 1-3:86-92. https://doi.org/10.1016/j.pmpp.2007.02.002

Gachomo EW, Kotchoni SO (2007) Detailed description of developmental growth stages of Diplocarpon rosae in Rosa: a core building block for efficient disease management. Ann Appl Biol 151:233-243. https://doi.org/10.1111/j.1744-7348.2007.00167.x

Gachomo EW, Seufferheld MJ, Kotchoni SO (2010) Melanization of appressoria is critical for the pathogenicity of Diplocarpon rosae. Mol Biol Rep 37:3583-3591. https://doi.org/10.1007/s11033-010-0007-4

Gartner GAL, McCouch SR, Moncada MDP (2013) A genetic map of an interspecific diploid pseudo testcross population of coffee. Euphytica 192:305-323. https://doi.org/10.1007/s10681-013-0926-y

Goffinet B, Gerber S (2000) Quantitative trait loci: a meta-analysis. Genetics 155:463-473

Grattapaglia D, Sederoff R (1994) Genetic linkage maps of Eucalyptus grandis and Eucalyptus urophylla using a pseudo-testcross: mapping strategy and RAPD markers. Genetics 137:1121-1137

Griffiths S, Simmonds J, Leverington M, et al (2009) Meta-QTL analysis of the genetic control of ear emergence in elite European winter wheat germplasm. TAG Theor Appl Genet Theor Angew Genet 119:383-395. https://doi.org/10.1007/s00122-009-1046-x

Guo B, Sleper DA, Lu P, et al (2006) QTLs associated with resistance to soybean cyst nematode in soybean: metaanalysis of QTL locations. Crop Sci

Guo J, Chen L, Li Y, et al (2018) Meta-QTL analysis and identification of candidate genes related to root traits in maize. Euphytica 214:223. https://doi.org/10.1007/s10681-018-2283-3

Hamon C, Coyne CJ, McGee RJ, et al (2013) QTL meta-analysis provides a comprehensive view of loci controlling partial resistance to Aphanomyces euteiches in four sources of resistance in pea. BMC Plant Biol 13:45. https://doi.org/10.1186/1471-2229-13-45

Harp DA, Zlesak DC, Hammond G, et al (2009) Earth-Kind® rose trials - identifying the world's strongest, most beautiful landscape roses. 10

Harville DA (1977) Maximum likelihood approaches to variance component estimation and to related problems. J Am Stat Assoc 72:320-338. https://doi.org/10.2307/2286796

Hattendorf A, Linde M, Mattiesch L, et al (2004) Genetic analysis of rose resistance genes and their localisation in the rose genome. Acta Hortic 123-130. https://doi.org/10.17660/ActaHortic.2004.651.14

Hibrand-Saint Oyant L, Crespel L, Rajapakse S, et al (2007) Genetic linkage maps of rose constructed with new microsatellite markers and locating QTL controlling flowering traits. Tree Genet Genomes 4:11. https://doi.org/10.1007/s11295-007-0084-2

Hibrand-Saint Oyant L, Ruttink T, Hamama L, et al (2018) A high-quality genome sequence of Rosa chinensis to elucidate ornamental traits. Nat Plants 4:473-484. https://doi.org/10.1038/s41477-018-0166-1 
Ho VSM, Wong JH, Ng TB (2007) A thaumatin-like antifungal protein from the emperor banana. Peptides 28:760766. https://doi.org/10.1016/j.peptides.2007.01.005

Holland JB, Coles ND (2011) QTL controlling masculinization of ear tips in a maize (zea mays 1.) intraspecific cross. G3 GenesGenomesGenetics 1:337-341. https://doi.org/10.1534/g3.111.000786

Holland JB, Nyquist WE, Cervantes-Martínez CT (2010) Estimating and interpreting heritability for plant breeding: an update. Plant Breed Rev 9-112. https://doi.org/10.1002/9780470650202.ch2

Horst RK, Cloyd RA (2007) Compendium of rose diseases and pests. APS Press

Kao C-H, Zeng M-H (2010) An investigation of the power for separating closely linked QTL in experimental populations. Genet Res 92:283-294. https://doi.org/10.1017/S0016672310000273

Kaufmann H, Mattiesch L, Lörz H, Debener T (2003) Construction of a BAC library of Rosa rugosa Thunb. and assembly of a contig spanning Rdr1, a gene that confers resistance to blackspot. Mol Genet Genomics 268:666-674. https://doi.org/10.1007/s00438-002-0784-0

Kaufmann H, Terefe D, Yasmin A, et al (2010) Cloning and analysis of Rdrl, a black spot resistance gene from roses. Acta Hortic 191-196. https://doi.org/10.17660/ActaHortic.2010.870.25

Kawamura K, Hibrand-Saint Oyant L, Crespel L, et al (2011) Quantitative trait loci for flowering time and inflorescence architecture in rose. TAG Theor Appl Genet Theor Angew Genet 122:661-675. https://doi.org/10.1007/s00122-010-1476-5

Kawamura K, Hibrand-Saint Oyant L, Thouroude T, et al (2015) Inheritance of garden rose architecture and its association with flowering behaviour. Tree Genet Genomes 11:1-12. https://doi.org/10.1007/s11295-0150844-3

Kelly JD, Vallejo V (2006) QTL analysis of multigenic disease resistance in plant breeding. In: Tuzun S, Bent E (eds) Multigenic and Induced Systemic Resistance in Plants. Springer US, Boston, MA, pp 21-48

Khowaja FS, Norton GJ, Courtois B, Price AH (2009) Improved resolution in the position of drought-related QTLs in a single mapping population of rice by meta-analysis. BMC Genomics 10:276. https://doi.org/10.1186/14712164-10-276

Kirst M, Myburg A, Sederoff R (2004) Genetic mapping in forest trees: markers, linkage analysis and genomics. In: Setlow JK (ed) Genetic Engineering: Principles and Methods. Springer US, Boston, MA, pp 105-141

Knight C (1975) Development of Diplocarpon rosae on different rose cultivars. PhD Thesis, London University

Krzywinski M, Schein J, Birol I, et al (2009) Circos: An information aesthetic for comparative genomics. Genome Res 19:1639-1645. https://doi.org/10.1101/gr.092759.109

Labbé J (2014) LOI n 2014-110 du 6 février 2014 visant à mieux encadrer l'utilisation des produits phytosanitaires sur le territoire national

Lacape J-M, Llewellyn D, Jacobs J, et al (2010) Meta-analysis of cotton fiber quality QTLs across diverse environments in a Gossypium hirsutum x G. barbadense RIL population. BMC Plant Biol 10:132. https://doi.org/10.1186/1471-2229-10-132

Lanaud C, Fouet O, Clément D, et al (2009) A meta-QTL analysis of disease resistance traits of Theobroma cacao L. Mol Breed 24:361-374. https://doi.org/10.1007/s11032-009-9297-4 
Lander ES, Botstein D (1989) Mapping mendelian factors underlying quantitative traits using RFLP linkage maps. Genetics 121:185-199

Lespinasse D, Rodier-Goud M, Grivet L, et al (2000) A saturated genetic linkage map of rubber tree (Hevea spp.) based on RFLP, AFLP, microsatellite, and isozyme markers. Theor Appl Genet 100:127-138. https://doi.org/10.1007/s001220050018

Leus L, Hosseini Moghaddam H, Van Huylenbroeck J, De Riek J (2015) QTLs associated with powdery mildew resistance responses in roses. Acta Hortic 287-293. https://doi.org/10.17660/ActaHortic.2015.1064.34

Liu S, Hall MD, Griffey CA, McKendry AL (2009) Meta-analysis of qtl associated with fusarium head blight resistance in wheat. Crop Sci 49:1955-1968. https://doi.org/10.2135/cropsci2009.03.0115

Lopez Arias DC, Chastellier A, Thouroude T, et al (in press) High density SNP and SSR linkage map and QTL analysis for resistance to black spot in segregating rose population. Acta Hortic

Lui S, Luo C, Zhu L, et al (2017) Identification and expression analysis of WRKY transcription factor genes in response to fungal pathogen and hormone treatments in apple (Malus domestica). J Plant Biol 60:215-230. https://doi.org/10.1007/s12374-016-0577-3

Lupas A, Van Dyke M, Stock J (1991) Predicting coiled coils from protein sequences. Science 252:1162-1164. https://doi.org/10.1126/science.252.5009.1162

Lynch M, Walsh B (1998) Genetics and analysis of quantitative traits. Sunderland, Mass. : Sinauer

Malek B von, Debener T (1998) Genetic analysis of resistance to blackspot (Diplocarpon rosae) in tetraploid roses. Theor Appl Genet 96:228-231. https://doi.org/10.1007/s001220050731

Malek B von, Weber WE, Debener T (2000) Identification of molecular markers linked to Rdr1 blackspot in roses. Theor Appl Genet 101:977-983. https://doi.org/10.1007/s001220051570

Marolleau B, Petiteau A, Bellanger MN, et al (2020) Strong differentiation within Diplocarpon rosae strains based on microsatellite markers and greenhouse-based inoculation protocol on Rosa. Plant Pathol

Menz I, Lakhwani D, Clotault J, et al (2020) Analysis of the Rdrl gene family in different Rosaceae genomes reveals an origin of an $R$-gene cluster after the split of Rubeae within the Rosoideae subfamily. PLOS ONE 15:e0227428. https://doi.org/10.1371/journal.pone.0227428

Menz I, Straube J, Linde M, Debener T (2018) The TNL gene Rdrl confers broad-spectrum resistance to Diplocarpon rosae. Mol Plant Pathol. https://doi.org/10.1111/mpp.12589

Meynet J (Institut N de la RA, Barrade R, Duclos A, Siadous R (1994) Dihaploid plants of roses (Rosa x hybrida, cv "Sonia") obtained by parthenogenesis induced using irradiated pollen and in vitro culture of immature seeds. Agron Fr

Morant M, Bak S, Møller BL, Werck-Reichhart D (2003) Plant cytochromes P450: tools for pharmacology, plant protection and phytoremediation. Curr Opin Biotechnol 14:151-162. https://doi.org/10.1016/S09581669(03)00024-7

Münnekhoff A-K, Linde M, Debener T (2017) The gene diversity pattern of Diplocarpon rosae populations is shaped by the age, diversity and fungicide treatment of their host populations. Plant Pathol 66:1288-1298. https://doi.org/10.1111/ppa.12681

Nakamichi R, Ukai Y, Kishino H (2001) Detection of closely linked multiple quantitative trait loci using a genetic algorithm. Genetics 158:463-475 
Niks RE, Qi X, Marcel TC (2015) Quantitative resistance to biotrophic filamentous plant pathogens: concepts, misconceptions, and mechanisms. Annu Rev Phytopathol 53:445-470. https://doi.org/10.1146/annurevphyto-080614-115928

Pandey SP, Somssich IE (2009) The Role of WRKY Transcription Factors in Plant Immunity. Plant Physiol 150:16481655. https://doi.org/10.1104/pp.109.138990

Patterson HD, Thompson R (1971) Recovery of inter-block information when block sizes are unequal. Biometrika 58:545-554. https://doi.org/10.2307/2334389

Pilet-Nayel M-L, Moury B, Caffier V, et al (2017) Quantitative resistance to plant pathogens in pyramiding strategies for durable crop protection. Front Plant Sci 8:1838. https://doi.org/10.3389/fpls.2017.01838

Poland JA, Balint-Kurti PJ, Wisser RJ, et al (2009) Shades of gray: the world of quantitative disease resistance. Trends Plant Sci 14:21-29. https://doi.org/10.1016/j.tplants.2008.10.006

Qi Z, Sun Y, Wang J, et al (2011) Meta-Analysis of 100-Seed Weight QTLs in Soybean. Agric Sci China 10:327-334. https://doi.org/10.1016/S1671-2927(11)60011-4

Rawat N (2016) Approaches for disease resistant candidate genes identification in plants: recent techniques and trends. Austin Food Sci 1:1010

Raymond O, Gouzy J, Just J, et al (2018) The Rosa genome provides new insights into the domestication of modern roses. Nat Genet 50:772. https://doi.org/10.1038/s41588-018-0110-3

Roman H, Rapicault M, Miclot AS, et al (2015) Genetic analysis of the flowering date and number of petals in rose. Tree Genet Genomes 11:85. https://doi.org/10.1007/s11295-015-0906-6

Roux F, Voisin D, Badet T, et al (2014) Resistance to phytopathogens e tutti quanti: placing plant quantitative disease resistance on the map. Mol Plant Pathol 15:427-432. https://doi.org/10.1111/mpp.12138

Said JI, Lin Z, Zhang X, et al (2013) A comprehensive meta QTL analysis for fiber quality, yield, yield related and morphological traits, drought tolerance, and disease resistance in tetraploid cotton. BMC Genomics 14:776. https://doi.org/10.1186/1471-2164-14-776

Saunders (1966) Epidemiological aspects of blackspot disease of roses caused by Diplocarpon rosae Wolf. Ann Appl Biol 58:115-122. https://doi.org/10.1111/j.1744-7348.1966.tb05076.x

Schuler MA, Duan H, Bilgin M, Ali S (2006) Arabidopsis cytochrome P450s through the looking glass: a window on plant biochemistry. Phytochem Rev 5:205-237. https://doi.org/10.1007/s11101-006-9035-z

Schuler MA, Werck-Reichhart D (2003) Functional genomics of P450s. Annu Rev Plant Biol 54:629-667. https://doi.org/10.1146/annurev.arplant.54.031902.134840

Schulz DF, Linde M, Blechert O, Debener T (2009) Evaluation of genus Rosa germplasm for resistance to black spot, downy mildew and powdery mildew. Eur J Hortic Sci 74:1-9

Semagn K, Beyene Y, Warburton ML, et al (2013) Meta-analyses of QTL for grain yield and anthesis silking interval in 18 maize populations evaluated under water-stressed and well-watered environments. BMC Genomics 14:313. https://doi.org/10.1186/1471-2164-14-313

Shupert DA (2006) Inheritance of flower, stem, leaf, and disease traits in three diploid interspecific rose populations. Texas A\&M University 
Singh NK, Kumar KRR, Kumar D, et al (2013) Characterization of a Pathogen Induced Thaumatin-Like Protein Gene AdTLP from Arachis diogoi, a Wild Peanut. PLOS ONE 8:e83963. https://doi.org/10.1371/journal.pone.0083963

Smith IM, Dunez J, Philips DH, et al (1989) European handbook of plant diseases. Q Rev Biol 64:200-200. https://doi.org/10.1086/416271

Smulders MJM, Arens P, Bourke PM, et al (2019) In the name of the rose: a roadmap for rose research in the genome era. Hortic Res 6:65-65. https://doi.org/10.1038/s41438-019-0156-0

Sosnowski O, Charcosset A, Joets J (2012) BioMercator V3: an upgrade of genetic map compilation and quantitative trait loci meta-analysis algorithms. Bioinforma Oxf Engl 28:2082-2083. https://doi.org/10.1093/bioinformatics/bts313

Soufflet-Freslon V, Marolleau B, Thouroude T, et al (2019) Development of tools to study rose resistance to black spot. Acta Hortic 213-220. https://doi.org/10.17660/ActaHortic.2019.1232.31

Spiller M, Linde M, Hibrand-Saint Oyant L, et al (2011) Towards a unified genetic map for diploid roses. Theor Appl Genet 122:489-500. https://doi.org/10.1007/s00122-010-1463-X

St Clair DA (2010) Quantitative disease resistance and quantitative resistance Loci in breeding. Annu Rev Phytopathol 48:247-268. https://doi.org/10.1146/annurev-phyto-080508-081904

Stamatakis A (2014) RAxML version 8: a tool for phylogenetic analysis and post-analysis of large phylogenies. Bioinformatics 30:1312-1313. https://doi.org/10.1093/bioinformatics/btu033

Terefe-Ayana D, Yasmin A, Le TL, et al (2011) Mining disease-resistance genes in roses: functional and molecular characterization of the Rdrl locus. Front Plant Sci 2:. https://doi.org/10.3389/fpls.2011.00035

Trognitz F, Manosalva P, Gysin R, et al (2002) Plant defense genes associated with quantitative resistance to potato late blight in Solanum phureja x dihaploid S. tuberosum hybrids. Mol Plant-Microbe Interact MPMI 15:587597. https://doi.org/10.1094/MPMI.2002.15.6.587

Tsuchiya T, Eulgem T (2011) EMSY-like genes are required for full RPP7-mediated race-specific immunity and basal defense in Arabidopsis. Mol Plant-Microbe Interact MPMI 24:1573-1581. https://doi.org/10.1094/MPMI05-11-0123

Uggla M, Carlson-Nilsson BU (2005) Screening of fungal diseases in offspring from crosses between Rosa sections Caninae and Cinnamomeae. Sci Hortic 104:493-504. https://doi.org/10.1016/j.scienta.2004.11.001

VAL'HOR, FranceAgriMer (2017) Bilan complet achats de végétaux

Van Ooijen JW (2011) Multipoint maximum likelihood mapping in a full-sib family of an outbreeding species. Genet Res 93:343-349. https://doi.org/10.1017/S0016672311000279

Vasconcellos RCC, Oraguzie OB, Soler A, et al (2017) Meta-QTL for resistance to white mold in common bean. PLOS ONE 12:e0171685. https://doi.org/10.1371/journal.pone.0171685

Veyrieras J-B, Goffinet B, Charcosset A (2007) MetaQTL: a package of new computational methods for the metaanalysis of QTL mapping experiments. BMC Bioinformatics 8:49. https://doi.org/10.1186/1471-2105-8-49

Voigt CA (2014) Callose-mediated resistance to pathogenic intruders in plant defense-related papillae. Front Plant Sci 5:. https://doi.org/10.3389/fpls.2014.00168 
Voigt CA (2016) Cellulose/callose glucan networks: the key to powdery mildew resistance in plants? New Phytol 212:303-305. https://doi.org/10.1111/nph.14198

Voigt CA, Somerville SC (2009) Chapter 4.4.5 - Callose in Biotic Stress (Pathogenesis): Biology, biochemistry and molecular biology of callose in plant defence: callose deposition and turnover in plant-pathogen interactions. In: Bacic A, Fincher GB, Stone BA (eds) Chemistry, Biochemistry, and Biology of 1-3 Beta Glucans and Related Polysaccharides. Academic Press, San Diego, pp 525-562

Waliczek TM, Byrne DH, Holeman DJ (2015a) Growers' and consumers' knowledge, attitudes and opinions regarding roses available for purchase. Acta Hortic

Waliczek TM, Byrne DH, Holeman DJ (2015b) Growers' and consumers' knowledge, attitudes and opinions regarding roses available for purchase. Acta Hortic 235-239. https://doi.org/10.17660/ActaHortic.2015.1064.26

Wang H, Yang Y, Li M, et al (2017) Residents' preferences for roses, features of rose plantings and the relations between them in built-up areas of Beijing, China. Urban For Urban Green 27:1-8. https://doi.org/10.1016/j.ufug.2017.06.011

Weber CA, Moore GA, Deng Z, Gmitter FG (2003) Mapping Freeze Tolerance Quantitative Trait Loci in a Citrus grandis $\times$ Poncirus trifoliata F1 Pseudo-testcross Using Molecular Markers. J Am Soc Hortic Sci 128:508514. https://doi.org/10.21273/JASHS.128.4.0508

Whitaker VM, Bradeen JM, Debener T, et al (2010a) Rdr3, a novel locus conferring black spot disease resistance in tetraploid rose: genetic analysis, LRR profiling, and SCAR marker development. TAG Theor Appl Genet Theor Angew Genet 120:573-585. https://doi.org/10.1007/s00122-009-1177-0

Whitaker VM, Debener T, Roberts AV, Hokanson SC (2010b) A standard set of host differentials and unified nomenclature for an international collection of Diplocarpon rosae races. Plant Pathol 59:745-752. https://doi.org/10.1111/j.1365-3059.2010.02281.x

Whitaker VM, Hokanson SC (2009) Partial resistance to black spot disease in diploid and tetraploid roses: general combining ability and implications for breeding and selection. Euphytica 169:421-429. https://doi.org/10.1007/s10681-009-9976-6

Whitaker VM, Hokanson SC, Bradeen J (2007) Distribution of Rose Black Spot (Diplocarpon rosae) Genetic Diversity in Eastern North America Using Amplified Fragment Length Polymorphism and Implications for Resistance Screening. J Am Soc Hortic Sci 132:534-540

White MA, Steffy B, Wiltshire T, Payseur BA (2011) Genetic dissection of a key reproductive barrier between nascent species of house mice. Genetics 189:289-304. https://doi.org/10.1534/genetics.111.129171

White MA, Stubbings M, Dumont BL, Payseur BA (2012) Genetics and evolution of hybrid male sterility in house mice. Genetics 191:917-934. https://doi.org/10.1534/genetics.112.140251

Wiggers RJ, West JG, Taylor J (1997) Conidial germination and infection by Diplocarpon rosae on susceptible and resistant rose species. Mycologia 89:103-108. https://doi.org/10.2307/3761178

Xue AG, Davidson CG (1998) Components of Partial Resistance to Black Spot Disease (Diplocarpon rosae Wolf) in Garden Roses. HortScience 33:96-99

Yadava SK, Arumugam N, Mukhopadhyay A, et al (2012) QTL mapping of yield-associated traits in Brassica juncea: meta-analysis and epistatic interactions using two different crosses between east European and Indian gene pool lines. TAG Theor Appl Genet Theor Angew Genet 125:1553-1564. https://doi.org/10.1007/s00122-0121934-3 
1014

\section{List of abbreviation}

1016 018-1297-2

Yagi M, Kimura T, Yamamoto T, et al (2012) QTL analysis for resistance to bacterial wilt (Burkholderia caryophylli) in carnation (Dianthus caryophyllus) using an SSR-based genetic linkage map. Mol Breed 30:495-509. https://doi.org/10.1007/s11032-011-9639-x

Yagi M (National I of FS, Onozaki T, Taneya M, et al (2006) Construction of a genetic linkage map for the carnation (Dianthus caryophyllus by using RAPD and SSR markers and mapping quantitative trait loci (QTL) for resistance to bacteria wilt caused by Burkholderia caryophylli. J Jpn Soc Hortic Sci Jpn

Yan M, Byrne DH, Klein PE, et al (2019) Black spot partial resistance in diploid roses: QTL discovery and linkage map creation. Acta Hortic 1232:135-141. https://doi.org/10.17660/ActaHortic.2019.1232.21

Yang B, Jiang Y, Rahman MH, et al (2009) Identification and expression analysis of WRKY transcription factor genes in canola (Brassica napus L.) in response to fungal pathogens and hormone treatments. BMC Plant Biol 9:68. https://doi.org/10.1186/1471-2229-9-68

Yang Q, Balint-Kurti P, Xu M (2017) Quantitative disease resistance: dissection and adoption in maize. Mol Plant 10:402-413. https://doi.org/10.1016/j.molp.2017.02.004

Yokoya K, Kandasamy KI, Walker S, et al (2000) Resistance of roses to pathotypes of Diplocarpon rosae. Ann Appl Biol 136:15-20. https://doi.org/10.1111/j.1744-7348.2000.tb00003.x

Zhang J, Wang F, Liang F, et al (2018) Functional analysis of a pathogenesis-related thaumatin-like protein gene TaLr35PR5 from wheat induced by leaf rust fungus. BMC Plant Biol 18:76. https://doi.org/10.1186/s12870-

Zhu M, Zhao S (2007) Candidate gene identification approach: progress and challenges. Int J Biol Sci 3:420-427

Zlesak DC, Nelson R, Harp D, et al (2017) Performance of landscape roses grown with minimal input in the northcentral, central, and south-central united states. HortTechnology 27:718-730. https://doi.org/10.21273/HORTTECH03681-17

Zlesak DC, Whitaker VM, George S, Hokanson SC (2010) Evaluation of roses from the Earth-Kind® trials: black spot (Diplocarpon rosae wolf) resistance and ploidy. HortScience 45:1779-1787. https://doi.org/10.21273/HORTSCI.45.12.1779

Zurn JD, Zlesak DC, Holen M, et al (2018) Mapping a novel black spot resistance locus in the climbing rose Brigth eyesTM ('radbrite'). Front Plant Sci 9:1730. https://doi.org/10.3389/fpls.2018.01730

Zurn JD, Zlesak DC, Holen M, et al (2020) Mapping the black spot resistance locus $R d r 3$ in the shrub rose 'George Vancouver' allows for the development of improved diagnostic markers for DNA-informed breeding. Theor Appl Genet. https://doi.org/10.1007/s00122-020-03574-4

Black Spot Disease (BSD); Quantitative Trait Loci (QTLs); Marker Assisted Breeding (MAS); Linkage Groups (LG); Rosa wichurana (RW); Rosa chinensis 'Old blush' (OB); Rosa hybrid 'The Fairy' (TF); Rosa chinensis 'Old blush' x Rosa wichurana population (OB population); Rosa hybrid 'The Fairy' x Rosa wichurana population (FW population); H190 x Rosa wichurana population (HW population); Analysis of Variance (ANOVA); Restricted 
1020 Maximum Likelihood (REML); 'Nearest Neighbor fit' (N.N fit); Short Sequence Repeat (SSR); Single Nucleotide

1021 Polymorphism (SNP); Logarithm of Odds (LOD); Weighted Least Square (WLS); 'Akaike' Information Criterion

1022 (AIC); Confidence Intervals (CI); Centimorgans (cM); Gene Ontology (GO); Resistance gene (R-gene); Number of

1023 Petals (NP); Simple Interval Mapping (SIM); Composite Interval Mapping (CIM); Two-part model (2p model); TIR-

1024 NB-LRR (TNL); NB-encoding (Nucleotide Binding); Quantitative Disease Resistance (QDR); Quantitative resistance

1025 loci (QRL); Cytochromes P450 monooxygenases (CYP); Pathogenesis-Related (PR); Thaumatin-Like Protein (TLP) 


\section{Figure legends}

1028 Fig. 1 Black spot disease scoring data distribution for all years and locations for the three populations

1029 A: Scoring data for different years for OW population in Angers, B: Scoring data for different years for HW population

1030 in Angers, C: Scoring data for different years for HW population in Bellegarde, D: Scoring data for different years for 1031 HW population in Diémoz, and E: Scoring data for different years for FW population in Angers.

1032 

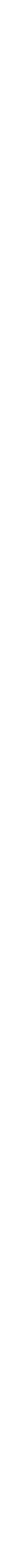

H190-NA
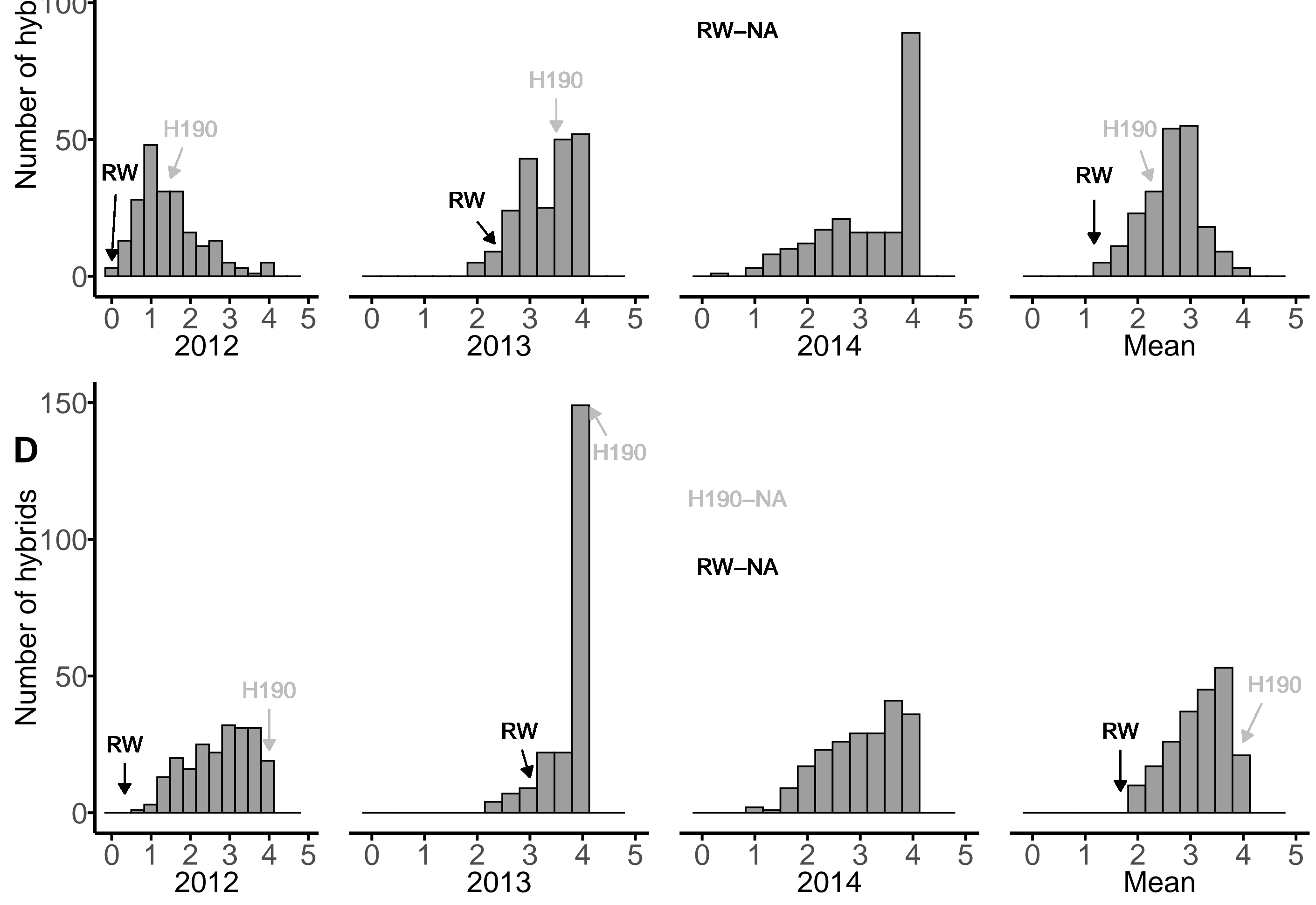

H190-NA

RW-NA
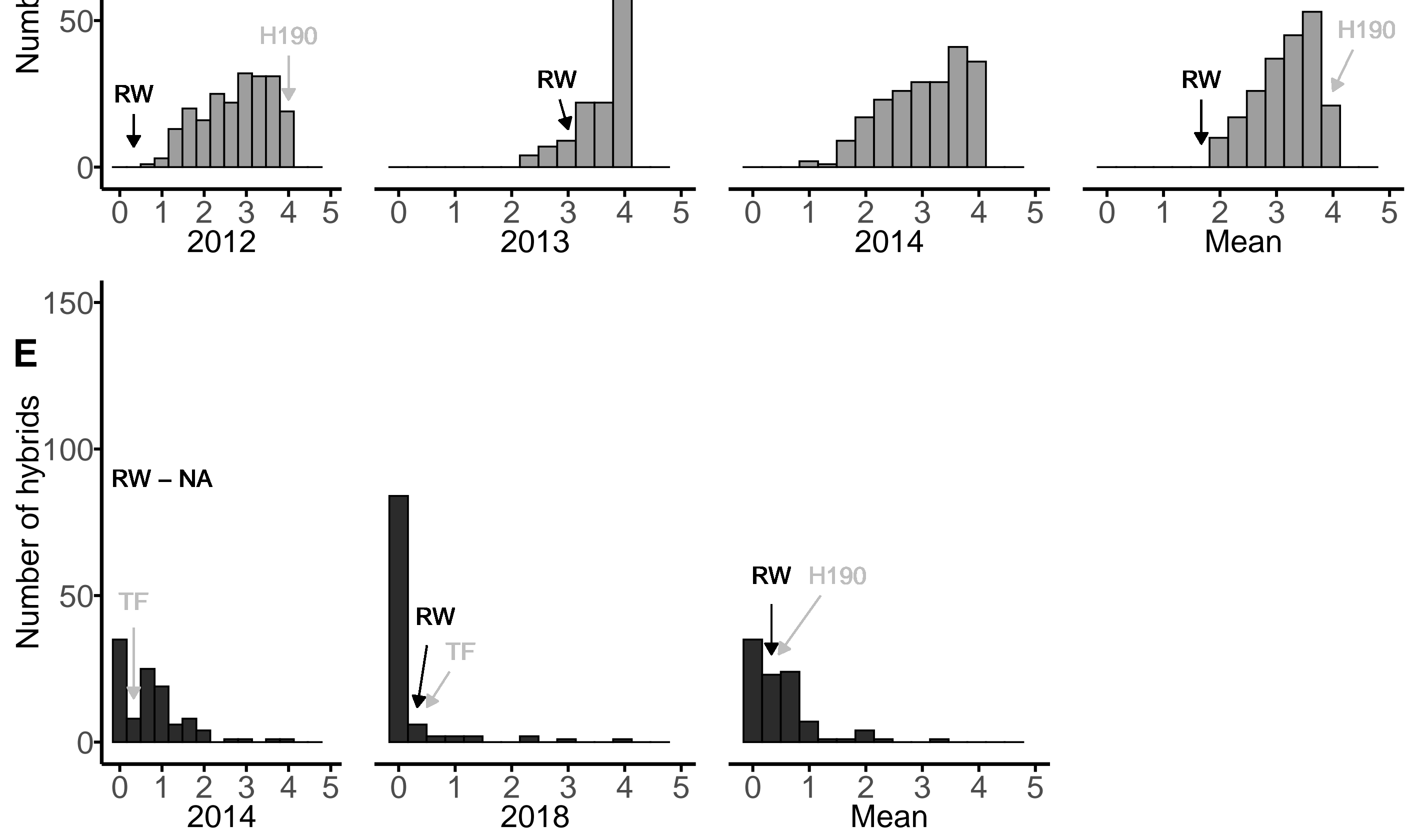
1033 Fig. 2 Representation of individual QTLs and meta-QTLs associated with black spot disease resistance for OW, FW 1034 and HW rose populations on the male consensus map

1035 Names of markers are on the right and the genetic distances (in cM) on the left. The 19 and 17 QTLs detected on B3 1036 and B5, respectively, on all three populations are projected onto a newly built consensus map. QTL names are coded 1037 as follows: PopName_map_YearLG_Location_method.95\% Bayesian confidence intervals are displayed with vertical 1038 bars where the length is proportional to the interval width, and the QTL peak is represented by a line. Meta-QTLs are 1039 represented in plain color on the LGs.

1040 

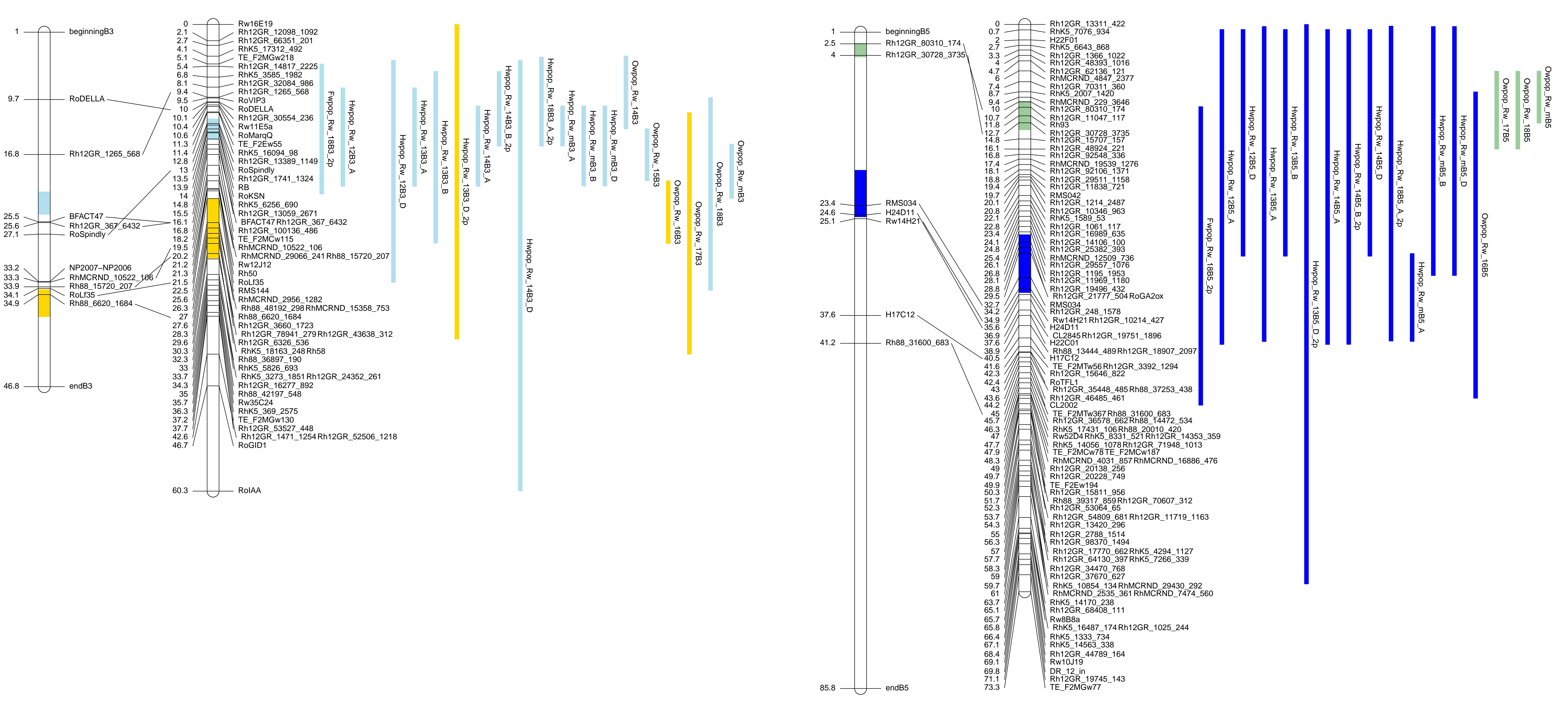
1041 Fig. 3 Candidate R-genes placed on the pseudochromosomes of Rosa chinensis assembly and meta-QTL position

1042 Each pseudochromosome is represented by a yellow bar, with the positions of the $R$-genes indicated as blue lines. 


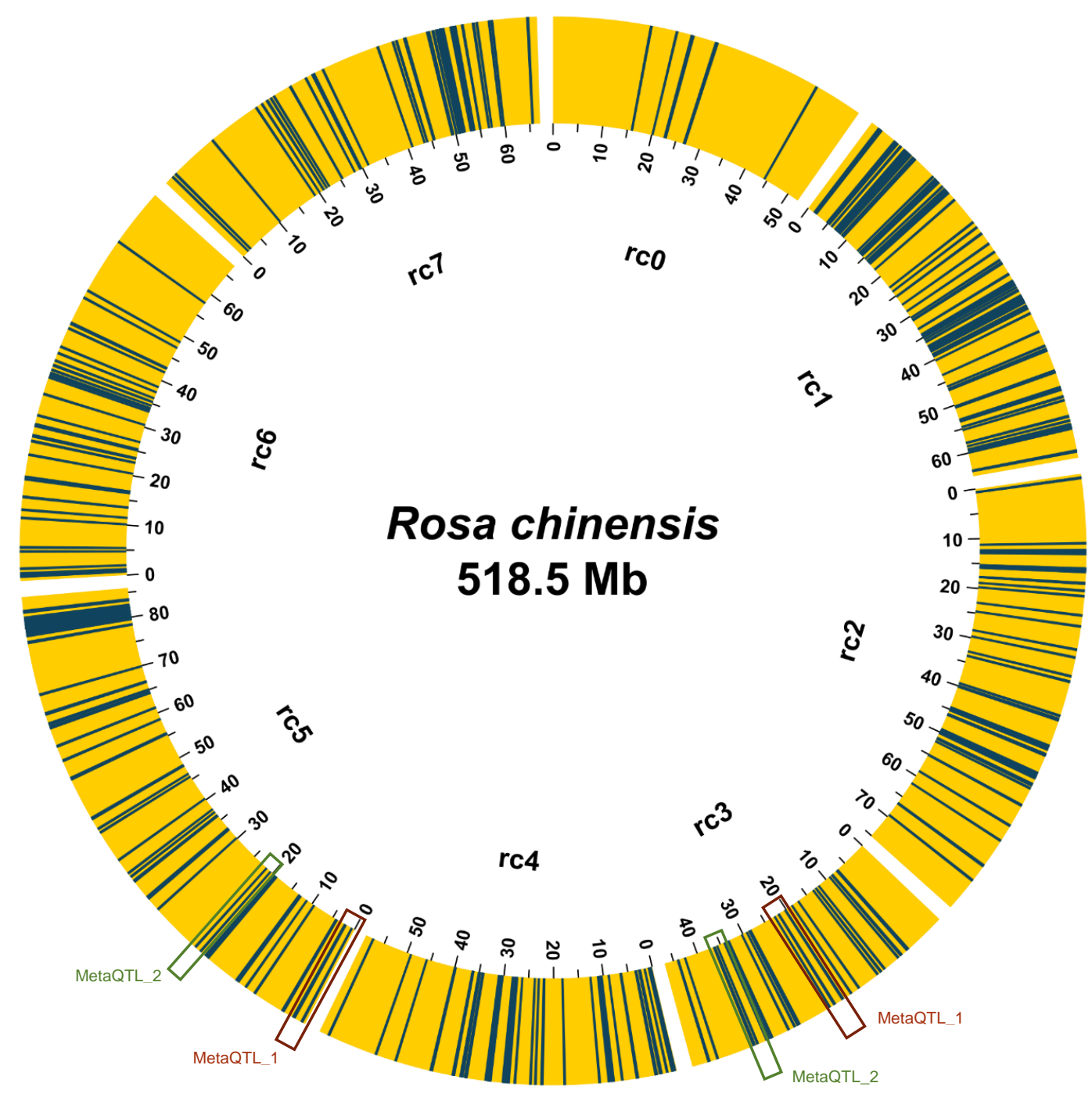




\section{Table legends}

1045 Table 1 Summary of the SSR and SNP-based linkage maps for OW population and the common markers with other 1046 populations

1047 Linkage groups (LG) names for the female map (A1 to A7) and for the male map (B1 to B7) were assigned according 1048 to Spiller et al. 2011. Several markers were mapped at the same locus so the number of unique loci is displayed and in 1049 the total number, the number of markers with different phases (called identicals) are displayed in parenthesis. One

1050 gene marker was used for the female map and is represented with $(+1)$ on the table. *Marker was counted as common 1051 when at minimum two of the three populations shared this same marker. 
Table 1 Summary of the SSR and SNP-based linkage maps for OW population and the common markers with other populations

\begin{tabular}{|c|c|c|c|c|c|c|c|c|c|c|c|c|c|c|c|}
\hline \multirow[b]{3}{*}{ LG } & \multicolumn{7}{|c|}{ Female map } & \multirow[b]{3}{*}{ LG } & \multicolumn{7}{|c|}{ Male map } \\
\hline & \multirow[b]{2}{*}{$\begin{array}{l}\text { Length } \\
\text { (cM) }\end{array}$} & \multicolumn{3}{|c|}{ Number of markers } & \multirow[b]{2}{*}{$\begin{array}{l}\text { Number } \\
\text { of loci }\end{array}$} & \multirow{2}{*}{$\begin{array}{c}\text { Average } \\
\text { marker } \\
\text { interval } \\
\text { (cM) }\end{array}$} & \multirow{2}{*}{$\begin{array}{l}\text { Number } \\
\text { of } \\
\text { common } \\
\text { markers* }\end{array}$} & & \multirow[b]{2}{*}{$\begin{array}{l}\text { Length } \\
\text { (cM) }\end{array}$} & \multicolumn{3}{|c|}{ Number of markers } & \multirow[b]{2}{*}{$\begin{array}{l}\text { Number } \\
\text { of loci }\end{array}$} & \multirow{2}{*}{$\begin{array}{c}\text { Average } \\
\text { marker } \\
\text { interval } \\
\text { (cM) }\end{array}$} & \multirow{2}{*}{$\begin{array}{c}\text { Number } \\
\text { of } \\
\text { common } \\
\text { markers* }\end{array}$} \\
\hline & & SNP & SSR & $\begin{array}{c}\text { Total } \\
\text { (identicals) }\end{array}$ & & & & & & $\begin{array}{c}S N \\
P\end{array}$ & SSR & $\begin{array}{c}\text { Total } \\
\text { (identicals) }\end{array}$ & & & \\
\hline A1 & 71.71 & 115 & 2 & $117(690)$ & 79 & 0.91 & 3 & B1 & 57.44 & 66 & 2 & $68(171)$ & 50 & 1.15 & 3 \\
\hline $\mathrm{A} 2$ & 33.42 & 69 & 3 & $72(1100)$ & 45 & 0.74 & 8 & B2 & 84.34 & 90 & 7 & $97(266)$ & 65 & 1.30 & 8 \\
\hline A3 & 53.75 & 61 & $5(+1)$ & $68(520)$ & 53 & 1.01 & 5 & B3 & 42.6 & 37 & 5 & $42(518)$ & 35 & 1.22 & 9 \\
\hline A4 & 58.79 & 41 & 5 & $46(220)$ & 40 & 1.47 & 4 & B4 & 66.82 & 73 & 7 & $80(371)$ & 66 & 1.01 & 6 \\
\hline A5 & 97.68 & 148 & 7 & 155 (1007) & 115 & 0.85 & 5 & B5 & 71.12 & 83 & 7 & $90(318)$ & 74 & 0.96 & 8 \\
\hline A6 & 70.15 & 133 & 4 & 137 (1134) & 91 & 0.77 & 3 & B6 & 55.89 & 65 & 3 & $68(210)$ & 48 & 1.16 & 3 \\
\hline A7 & 88.36 & 126 & 5 & $131(841)$ & 90 & 0.98 & 3 & B7 & 75.41 & 83 & 5 & 88 (217) & 64 & 1.18 & 4 \\
\hline Total & 473.85 & 692 & $31(+1)$ & $726(5512)$ & 513 & 0.96 & 31 & Total & 453.62 & 497 & 36 & $534(2072)$ & 402 & 1.14 & 41 \\
\hline
\end{tabular}

Linkage groups (LG) names for the female map (A1 to A7) and for the male map (B1 to B7) were assigned according to Spiller et al. 2011. Several makers were mapped at the same locus so the number of unique loci is displayed and in the total number, the number of markers with different phases (called identicals) are displayed in parenthesis. One gene marker was used for the female map and is represented with $(+1)$ on the table.

*Marker was count as common when minimum two of the three populations shared this same marker. 
1053 Table 2 Summary of QTL for black spot disease resistance in OW, FW and HW populations across multiple 1054 environments (years and locations) for male maps

1055 a Normal indicates a QTL mapping using a normal model with CIM analysis and 2p indicates a two-part model that 1056 studies consecutively a binary model and a normal one, so three LOD were calculated: LOD. $\pi$ (penetrance, equivalent

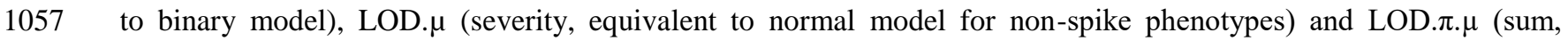
1058 complete model). ${ }^{\mathrm{b}}$ Permutation test giving the LOD threshold calculated using 1,000 permutations over which a QTL 1059 was significant. ${ }^{\mathrm{c}}$ Linkage group number with B for the male map. ${ }^{\mathrm{d}}$ Proportion of phenotypic variation explained by 1060 the QTL. ${ }^{\text {e }}$ Confidence interval calculated with the 95\% Bayesian credible interval.

1061

1062 
Table 2 Summary of QTL for black spot disease resistance in OW, FW and HW populations across multiple environments (years and locations) for male maps

\begin{tabular}{|c|c|c|c|c|c|c|c|c|c|c|c|c|c|}
\hline \multirow{2}{*}{ Population } & \multirow{2}{*}{ Methoda $^{a}$} & \multirow{2}{*}{ Location } & \multirow{2}{*}{ Year } & \multirow{2}{*}{$\mathrm{PT}^{\mathrm{b}}$} & \multirow{2}{*}{$\mathrm{LG}^{\mathrm{c}}$} & \multirow{2}{*}{$\mathrm{LOD}^{\mathrm{a}}$} & \multirow{2}{*}{ Lod. $\pi^{\mathrm{a}}$} & \multirow{2}{*}{ Lod. $\mu^{\mathrm{a}}$} & \multirow{2}{*}{$R^{2}(\%)^{d}$} & \multicolumn{2}{|c|}{$95 \%$ Bayes $\mathrm{Cl}$ (in cM) e } & \multicolumn{2}{|l|}{ Peak } \\
\hline & & & & & & & & & & Interval start & Interval end & Closest marker & Position (cM) \\
\hline \multirow[t]{12}{*}{ OW } & normal & Angers & 2014 & 2.6 & B3 & 3.16 & & & 10.1 & 4.07 & 13.5 & Rh12GR_1265_568 & 9.34 \\
\hline & & & 2015 & 2.6 & B3 & 8.72 & & & 23.6 & 13.5 & 20.2 & Rh12GR_367_6432 & 1613 \\
\hline & & & 2016 & 2.5 & B3 & 6.79 & & & 17.9 & 20.2 & 28.3 & Rh88_6620_1684 & 26.96 \\
\hline & & & & & B5 & 4.11 & & & 9.8 & 8.71 & 48.3 & Rh88_31600_683 & 44.99 \\
\hline & & & 2017 & 2.6 & B3 & 4.65 & & & 10.2 & 11.4 & 42.6 & Rh88_15720_207 & 20.19 \\
\hline & & & & & B4 & 3.55 & & & 7.6 & 2 & 65.4 & Rh12GR_14039_541 & 14.2 \\
\hline & & & & & B5 & 5.26 & & & 11.4 & 6.03 & 16.1 & Rh12GR_80310_174 & 10.04 \\
\hline & & & 2018 & 2.6 & B3 & 3.17 & & & 8.8 & 9.44 & 34.3 & RhMCRND_10522_106 & 19.52 \\
\hline & & & & & B5 & 3.43 & & & 9.3 & 6.03 & 16.1 & Rh12GR_80310_174 & 10.04 \\
\hline & & & Mean & 2.6 & B3 & 11.18 & & & 22.1 & 15.46 & 22.49 & Rh12GR_367_6432 & 16.33 \\
\hline & & & & & B4 & 3.02 & & & 5.1 & 13.95 & 23.6 & Rh88_49087_478 & 20.9 \\
\hline & & & & & B5 & 5.42 & & & 11 & 6.03 & 12.74 & Rh12GR_30728_3735 & 8.71 \\
\hline \multirow[t]{2}{*}{ FW } & $2 p$ & Angers & 2018 & 2.15 & B3 & 4.74 & 4.13 & 0.60 & 11.44 & 0 & 16.8 & BFACT47 & 7.01 \\
\hline & & & & & B5 & 2.91 & 2.74 & 0.17 & 0.89 & 10.4 & 49 & RoRGA2 & 29.34 \\
\hline \multirow[t]{13}{*}{$\mathrm{HW}$} & normal & Angers & 2012 & 2.32 & B3 & 7.67 & & & 15.74 & 3.97 & 16.7 & RB & 9.67 \\
\hline & & & & & B5 & 3.6 & & & 6.23 & 0 & 40.7 & Rw14H21 & 25.13 \\
\hline & & & 2013 & 2.21 & B3 & 8.56 & & & 16.02 & 3.97 & 16.7 & $\mathrm{RB}$ & 9.67 \\
\hline & & & & & B5 & 9.94 & & & 14.82 & 0 & 40.7 & RMSO34 & 32.18 \\
\hline & & & & & B6 & 2.87 & & & 3.6 & 0 & 62.5 & CTG623 & 8.28 \\
\hline & & & 2014 & 2.23 & B3 & 7.54 & & & 12.99 & 6.3 & 16.7 & RB & 9.67 \\
\hline & & & & & B5 & 6.36 & & & 9.66 & 0 & 40.7 & RMSO34 & 25.13 \\
\hline & & & & & B6 & 3.12 & & & 4.22 & 0 & 20.88 & CTG623 & 8.28 \\
\hline & & & Mean & 2.14 & B3 & 15.23 & & & 21.43 & 6.3 & 11.5 & $\mathrm{RB}$ & 9.67 \\
\hline & & & & & B5 & 12.14 & & & 16.76 & 29.34 & 40.69 & RMS034 & 32.18 \\
\hline & & & & & B6 & 3.92 & & & 4.63 & 0 & 20.88 & CTG623 & 8.28 \\
\hline & & Bellegarde & 2013 & 2.30 & B3 & 5.08 & & & 8.52 & 0 & 22.2 & RoRGA/RoDELLA & 3.97 \\
\hline & & & & & B5 & 9.86 & & & 19.6 & 0 & 29.3 & Rw14H21 & 25.13 \\
\hline
\end{tabular}


Table 2 (continued)

\begin{tabular}{|c|c|c|c|c|c|c|c|c|c|c|c|c|c|}
\hline \multirow{2}{*}{ Population } & \multirow{2}{*}{ Methoda } & \multirow{2}{*}{ Location } & \multirow{2}{*}{ Year } & \multirow{2}{*}{$\mathrm{PT}^{\mathrm{b}}$} & \multirow{2}{*}{$\mathrm{LG}^{\mathrm{c}}$} & \multirow{2}{*}{$\operatorname{LOD}^{\mathrm{a}}$} & \multirow{2}{*}{ Lod. $\pi^{\mathrm{a}}$} & \multirow{2}{*}{ Lod. $\mu^{\mathrm{a}}$} & \multirow{2}{*}{$R^{2}(\%)^{d}$} & \multicolumn{2}{|c|}{ 95\% Bayes $\mathrm{Cl}$ (in cM) e } & \multicolumn{2}{|c|}{ Peak } \\
\hline & & & & & & & & & & Interval start & Interval end & Closest marker & Position (cM) \\
\hline \multirow[t]{18}{*}{ HW } & normal & Bellegarde & Mean & 2.33 & B3 & 5.23 & & & 8.41 & 6.3 & 16.66 & RoMarQ & 6.3 \\
\hline & & & & & B5 & 4.99 & & & 5.35 & 0 & 32.18 & $\mathrm{Rw} 14 \mathrm{H} 21$ & 25.13 \\
\hline & & & & & B6 & 2.6 & & & 4.15 & 0 & 20.88 & CTG623 & 8.28 \\
\hline & & Diémoz & 2012 & 2.25 & B3 & 3.72 & & & 7.19 & 0 & 28.7 & $\mathrm{Rw} 14 \mathrm{H} 21$ & 25.13 \\
\hline & & & & & B5 & 4.21 & & & 9.14 & 0 & 29.3 & CTG623 & 8.28 \\
\hline & & & 2014 & 2.21 & B3 & 2.73 & & & 3.9 & 0 & 60.6 & BFACT47 & 11.5 \\
\hline & & & & & B5 & 4.64 & & & 8.72 & 0 & 29.3 & $\mathrm{Rw} 14 \mathrm{H} 21$ & 25.13 \\
\hline & & & & & B6 & 2.96 & & & 4.17 & 0 & 38.3 & CTG623 & 8.28 \\
\hline & & & Mean & 2.08 & B3 & 5.55 & & & 9.7 & 6.3 & 16.66 & BFACT47 & 11.5 \\
\hline & & & & & B5 & 8.43 & & & 12.78 & 0 & 32.18 & Rw14H21 & 25.13 \\
\hline & $2 p$ & Angers & 2018 & 3.03 & B3 & 8.19 & 0.77 & 7.42 & 15.66 & 0 & 11.5 & RoSpindly & 8.96 \\
\hline & & & & & B5 & 9.92 & 0.7 & 9.22 & 19.82 & 0 & 40.69 & H24D11 & 30.08 \\
\hline & & & & & B6 & 3.13 & 0.64 & 2.48 & 2.35 & 0 & 62.5 & CTG623 & 8.28 \\
\hline & & Bellegarde & 2014 & 2.96 & B3 & 12.55 & 9.62 & 2.95 & 13.69 & 0 & 9.67 & RoRGA & 3.97 \\
\hline & & & & & B5 & 6.39 & 4.84 & 1.59 & 8.17 & 0 & 40.69 & Rw14H21 & 25.13 \\
\hline & & & & & B6 & 4.87 & 4.7 & 0.17 & 2.76 & 0 & 20.88 & CTG623 & 8.28 \\
\hline & & Diémoz & 2013 & 3.06 & B3 & 4 & 0.87 & 3.13 & 8.96 & 0 & 60.63 & RoLf35 & 19.08 \\
\hline & & & & & B5 & 5.98 & 0.16 & 5.82 & 11.93 & 0 & 72.49 & H17C12 & 40.69 \\
\hline
\end{tabular}

${ }^{a}$ Normal indicates a QTL mapping using a normal model with CIM analysis and 2p indicates a two-part model that studies consecutively a binary model and a normal one, so three LOD were calculated: LOD. $\pi$ (penetrance, equivalent to binary model), LOD. $\mu$ (severity, equivalent to normal model for non-spike phenotypes) and LOD. $\pi . \mu$ (sum, complete model).

${ }^{\mathrm{b}}$ Permutation test giving the LOD threshold calculated using 1,000 permutations over which a QTL was significant.

${ }^{c}$ Linkage group number with B for the male map.

${ }^{\mathrm{d}}$ Proportion of phenotypic variation explained by the QTL.

${ }^{\mathrm{e}}$ Confidence interval calculated with the $95 \%$ Bayesian credible interval. 
1063 Table 3 Summary of QTL for black spot disease resistance in OW, FW and HW populations across multiple 1064 environments (years and locations) for female maps

$1065{ }^{a}$ Normal indicates a QTL mapping using a normal model with CIM analysis and 2p indicates a two-part model that 1066 studies consecutively a binary model and a normal one, so three LOD were calculated: LOD. $\pi$ (penetrance, equivalent

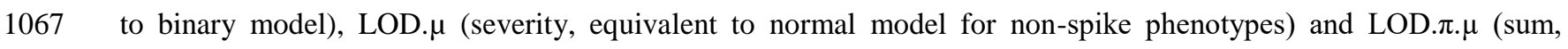
1068 complete model). ${ }^{\mathrm{b}}$ Permutation test giving the LOD threshold calculated using 1,000 permutations over which a QTL 1069 was significant. ${ }^{\mathrm{c}}$ Linkage group number with B for the male map. ${ }^{\mathrm{d}}$ Proportion of phenotypic variation explained by 1070 the QTL. ${ }^{\text {e }}$ Confidence interval calculated with the 95\% Bayesian credible interval. 
Table 3 Summary of QTL for black spot disease resistance in OW, FW and HW populations across multiple environments (years and locations) for female maps

\begin{tabular}{|c|c|c|c|c|c|c|c|c|c|c|c|c|c|}
\hline \multirow[t]{2}{*}{ Population } & \multirow[t]{2}{*}{ Method $^{a}$} & \multirow[t]{2}{*}{ Location } & \multirow[t]{2}{*}{ Year } & \multirow[t]{2}{*}{$\mathrm{PT}^{\mathrm{b}}$} & \multirow[t]{2}{*}{$L^{c}{ }^{c}$} & \multirow[t]{2}{*}{$\operatorname{LOD}^{a}$} & \multirow[t]{2}{*}{ Lod. $\pi^{\mathrm{a}}$} & \multirow[t]{2}{*}{ Lod. $\mu^{a}$} & \multirow[t]{2}{*}{$R^{2}(\%)^{d}$} & \multicolumn{2}{|c|}{$95 \%$ Bayes $\mathrm{Cl}$ (in cM) e } & \multicolumn{2}{|c|}{ Peak } \\
\hline & & & & & & & & & & Interval Start & Interval End & Nearest marker & Position (cM) \\
\hline OW & normal & Angers & 2017 & 2.71 & A1 & 4.12 & & & 6.53 & 42.89 & 60.3 & Rh12GR_100474_250 & 54.3 \\
\hline \multirow[t]{3}{*}{ FW } & $2 p$ & Angers & 2018 & 2.10 & A1 & 2.6 & 2.59 & 0.01 & 4.06 & 15.92 & 44.87 & Rw34L6 & 35 \\
\hline & & & & & $\mathrm{A} 2$ & 5.86 & 3.16 & 2.63 & 2.57 & 1.12 & 14.64 & Rw59A12 & 1.12 \\
\hline & & & & & A4 & 4.18 & 4.09 & 0.01 & 3.61 & 32.53 & 44.28 & Rw53021 & 44.28 \\
\hline \multirow[t]{8}{*}{ HW } & normal & Angers & 2012 & 2.04 & A3 & 2.77 & & & 5.8 & 23.73 & 41.37 & NP2007 & 33.71 \\
\hline & & Angers & 2014 & 2.09 & A3 & 2.3 & & & 4.44 & 23.73 & 41.37 & NP2007 & 33.71 \\
\hline & & Bellegarde & 2012 & 2.05 & A5 & 2.51 & & & 5.39 & 0 & 36.04 & H24D11 & 27.09 \\
\hline & & Bellegarde & 2013 & 1.95 & A3 & 2.47 & & & 5.1 & 12.35 & 33.71 & RoLf35H & 23.73 \\
\hline & & Diémoz & 2014 & 2.08 & A3 & 2.63 & & & 5.74 & 23.73 & 41.37 & NP2007 & 33.71 \\
\hline & & & & & A4 & 2.87 & & & 5.77 & 0 & 37.36 & Rw20l17 & 12.6 \\
\hline & & Diémoz & Mean & 2.09 & A3 & 2.17 & & & 4.4 & 23.73 & 41.37 & NP2007 & 33.71 \\
\hline & $2 p$ & Bellegarde & 2014 & 2.73 & A3 & 3.02 & 2.15 & 0.91 & 5.91 & 12.35 & 51.19 & NP2006 & 33.71 \\
\hline
\end{tabular}

${ }^{a}$ Normal indicates a QTL mapping using a normal model with CIM analysis and 2p indicates a two-part model that studies consecutively a binary model and a normal one, so three LOD were calculated: LOD. $\pi$ (penetrance, equivalent to binary model), LOD. $\mu$ (severity, equivalent to normal model for non-spike phenotypes) and LOD. $\pi . \mu$ (sum, complete model).

${ }^{\mathrm{b}}$ Permutation test giving the LOD threshold calculated using 1,000 permutations over which a QTL was significant.

${ }^{\mathrm{c}}$ Linkage group number with $\mathrm{B}$ for the male map.

${ }^{\mathrm{d}}$ Proportion of phenotypic variation explained by the QTL.

${ }^{\mathrm{e}}$ Confidence interval calculated with the $95 \%$ Bayesian credible interval. 
1073 Table 4 Meta-QTL genomic position and gene mining

$1074 \quad{ }^{a}$ Meta-QTL names using all the QTLs found with the normal and $2 \mathrm{p}$ method results. ${ }^{\mathrm{b}}$ Chromosome numbers from 1075 Rosa chinensis genome (Hibrand Saint-Oyant et al. 2018). ${ }^{\mathrm{c}}$ Genomic positions of the meta-QTLs after projection on 1076 Rosa chinensis genome (Hibrand Saint-Oyant et al. 2018). ${ }^{\mathrm{d}}$ Genes coding for disease resistance proteins from the 1077 automatic annotation of the genome. ${ }^{\mathrm{e}}$ Resistance gene analogs with $N B$-domain found with protein sequence scan 1078 (manual annotation). ${ }^{\mathrm{f}}$ Total number of annotated genes under meta-QTL intervals with Goffinet et al. (2000) method. 1079

1080 
Table 4 Meta-QTL genomic position and gene mining

\begin{tabular}{|c|c|c|c|c|c|c|c|c|}
\hline \multirow{2}{*}{ Meta_QTL ${ }^{a}$} & \multirow{2}{*}{$\mathrm{Chr}^{\mathrm{b}}$} & \multicolumn{2}{|c|}{$\begin{array}{c}\text { Genetic } \\
\text { position (cM) }\end{array}$} & \multicolumn{2}{|c|}{ Interval position $(b p)^{c}$} & \multicolumn{3}{|c|}{ Number of genes in the interval } \\
\hline & & start & end & start & end & RGAs_auto ${ }^{d}$ & RGAs_manuale & Total $^{f}$ \\
\hline Meta_1_3 & 3 & 12.17 & 14.89 & 21605249 & 24567362 & 11 & 4 & 291 \\
\hline Meta_2_3 & 3 & 22.51 & 30.21 & 34220246 & 37772912 & 4 & 3 & 450 \\
\hline Meta_1_5 & 5 & 9.68 & 13.63 & 2414969 & 4219224 & 3 & 3 & 271 \\
\hline Meta_2_5 & 5 & 27.14 & 34.66 & 18827666 & 24889549 & 5 & 9 & 557 \\
\hline
\end{tabular}

${ }^{a}$ Meta-QTL names using all the QTLs found with the normal and $2 p$ method results.

${ }^{\mathrm{b}}$ Chromosome numbers from Rosa chinensis genome (Hibrand Saint-Oyant et al. 2018).

${ }^{\mathrm{c}}$ Genomic positions of the meta-QTLs after projection on Rosa chinensis genome (Hibrand Saint-Oyant et al. 2018).

${ }^{\mathrm{d}}$ Genes coding for disease resistance proteins from the automatic annotation of the genome.

${ }^{\text {e }}$ Resistance gene analogs with $N B$-domain found with protein sequence scan (manual annotation).

${ }^{\mathrm{f}}$ Total number of annotated genes under meta-QTL intervals with Goffinet et al. (2000) method. 


\section{$1081 \quad$ Additional files}

\section{Supplementary tables}

1083 Supp. Table 1 Common marker list for all populations for the female and male maps

1084 a Only SSR markers are listed. The markers shared by all three populations are displayed in green; ${ }^{\mathrm{b}}$ A1-A7 for the 1085 female maps and B1-B7 for the male maps.

1086

1087 
Supp. table 1 Common marker list for all populations for the female and male maps

\begin{tabular}{|c|c|c|c|c|c|c|c|c|c|}
\hline \multicolumn{5}{|c|}{ Male maps } & \multicolumn{5}{|c|}{ Female maps } \\
\hline Markers $^{\mathrm{a}}$ & $\mathrm{LG}^{\mathrm{b}}$ & OW & $\mathrm{HW}$ & FW & Markers $^{\mathrm{a}}$ & $\mathrm{LG}^{\mathrm{b}}$ & OW & $\mathrm{HW}$ & $\mathrm{FW}$ \\
\hline RMS015 & $\mathrm{B} 1$ & $x$ & $x$ & $x$ & RMS015 & A1 & $x$ & & $x$ \\
\hline Rw25J16 & B1 & & $x$ & $x$ & Rw32K24 & $\mathrm{A} 1$ & $x$ & & $x$ \\
\hline Rw32K24 & B1 & $x$ & $x$ & $x$ & Rw25J16 & $\mathrm{A} 1$ & & $x$ & $x$ \\
\hline CTG329 & B2 & & $x$ & $x$ & $\underline{\text { RMS132 }}$ & $\mathrm{A} 2$ & $x$ & $x$ & $x$ \\
\hline CTG356 & $\mathrm{B} 2$ & $x$ & $x$ & & $\overline{\text { CTG356 }}$ & $\mathrm{A} 2$ & $x$ & & $x$ \\
\hline RMS129 & B2 & $x$ & & $x$ & RMS147 & $\mathrm{A} 2$ & & $x$ & $x$ \\
\hline RMS132 & B2 & $x$ & $x$ & $x$ & Rw59A12 & $\mathrm{A} 2$ & & $x$ & $x$ \\
\hline$\underline{\text { RMS137 }}$ & B2 & $x$ & $x$ & $x$ & Rw23F13 & $\mathrm{A} 2$ & & $x$ & $x$ \\
\hline RMS147 & B2 & $x$ & $x$ & $x$ & RMS137 & $\mathrm{A} 2$ & & $x$ & $x$ \\
\hline Rw23F13 & B2 & $x$ & & $x$ & CTG329 & $\mathrm{A} 2$ & & $x$ & $x$ \\
\hline Rw59A12 & B2 & $x$ & $x$ & $x$ & Rh80 & $\mathrm{A} 2$ & & $x$ & $x$ \\
\hline BFACT47 & B3 & $x$ & $x$ & $x$ & BFACT47 & A3 & $x$ & $x$ & \\
\hline$\overline{\text { RoAP2 }}$ & B3 & & $x$ & $x$ & Rh58 & $\mathrm{A} 3$ & $x$ & $x$ & $x$ \\
\hline Rh50 & B3 & & $x$ & $x$ & Rw35C24 & A3 & $x$ & & $x$ \\
\hline$\underline{\text { Rh58 }}$ & B3 & $x$ & $x$ & $x$ & CTG21 & $\mathrm{A} 3$ & $x$ & & $x$ \\
\hline RMS144 & B3 & $x$ & & $x$ & Rh50 & $\mathrm{A} 3$ & & $x$ & $x$ \\
\hline RolAA & B3 & & $x$ & $x$ & Rog5 & A4 & $x$ & $x$ & \\
\hline RoVIP3 & B3 & & $x$ & $x$ & Rw55E12 & A4 & $x$ & $x$ & \\
\hline$\underline{\text { Rw16E19 }}$ & B3 & $x$ & $x$ & $x$ & Cl3881 & A4 & $x$ & & $x$ \\
\hline Rw35C24 & B3 & $x$ & $x$ & $x$ & Rh98 & A4 & $x$ & $x$ & $x$ \\
\hline$\underline{\mathrm{Cl} 3881}$ & B4 & $x$ & $x$ & $x$ & H22F01 & A5 & $x$ & $x$ & \\
\hline$\overline{\mathrm{H} 20 \mathrm{D0} 0}$ & B4 & $x$ & $x$ & & H24D11 & A5 & $x$ & $x$ & \\
\hline H2F12 & B4 & $x$ & $x$ & $x$ & Rw14H21 & A5 & $x$ & $x$ & $x$ \\
\hline Rog5 & B4 & $x$ & & $x$ & CL2845 & A5 & $x$ & $x$ & $x$ \\
\hline Rw53021 & B4 & $x$ & $x$ & $x$ & Rw10J19 & A5 & $x$ & & $x$ \\
\hline$\underline{\text { Rw55E12 }}$ & B4 & $x$ & $x$ & $x$ & Rw22A3 & A6 & $x$ & & $x$ \\
\hline$\underline{\mathrm{CL} 2845}$ & B5 & $x$ & $x$ & $x$ & Rw61F2 & A6 & $x$ & & $x$ \\
\hline H17C12 & B5 & & $x$ & $x$ & CTG623 & A6 & $x$ & $x$ & $x$ \\
\hline $\mathrm{H} 22 \mathrm{CO} 1$ & B5 & $x$ & $x$ & & $\underline{\mathrm{RMSO03}}$ & A7 & $x$ & $x$ & $x$ \\
\hline$\underline{\mathrm{H} 22 \mathrm{~F} 01}$ & B5 & $x$ & $x$ & $x$ & Rw10M24 & A7 & $x$ & $x$ & \\
\hline H24D11 & B5 & $x$ & & $x$ & $\underline{R w 15 D 15}$ & A7 & $x$ & $x$ & $x$ \\
\hline RMS034 & B5 & $x$ & & $x$ & & & & & \\
\hline Rw10J19 & B5 & $x$ & $x$ & $x$ & & & & & \\
\hline Rw14H21 & B5 & $x$ & $x$ & $x$ & & & & & \\
\hline CTG623 & B6 & $x$ & $x$ & $x$ & & & & & \\
\hline Rw22A3 & B6 & $x$ & $x$ & $x$ & & & & & \\
\hline Rw61F2 & B6 & $x$ & $x$ & & & & & & \\
\hline H10D03 & B7 & $x$ & $x$ & & & & & & \\
\hline$\underline{\mathrm{RMSO03}}$ & B7 & $x$ & $x$ & $x$ & & & & & \\
\hline Rw10M24 & B7 & $x$ & & $x$ & & & & & \\
\hline$\underline{R w 15 D 15}$ & B7 & $x$ & $x$ & $x$ & & & & & \\
\hline
\end{tabular}

${ }^{\text {a }}$ Only SSR markers are listed, the markers shared by all three populations are displayed in emphasized green; ${ }^{\mathrm{b}} \mathrm{A} 1$ A7 for the female maps and B1-B7 for the male maps. 
1088 Supp. Table 2 NB-encoding genes information and position in Rose genome

1089 Summary of NB-encoding genes identified in manual annotations. Listed are gene ID names, chromosomal affiliation,

1090 end and start positions, classification $(\mathrm{CC}=$ Coiled Coil; LRR=Leucine Rich Repeat; NBS=Nucleotide Binding Site;

1091 TIR=Toll Interleukin Like Receptor); non-canonical domains (i.e., conserved protein domains that are atypical of

1092 NBS-LRR proteins); configuration of identified conserved NB-ARC subdomains; and detailed domain configurations,

1093 complete with reported e-values from a Pfam analysis.

1094

1095 


\begin{tabular}{|c|c|c|c|c|c|c|c|}
\hline Gene ID & Chromosome & Start & End & Classification & $\begin{array}{l}\text { canonical } \\
\text { domains }\end{array}$ & Configuration of subdomains within NB-ARC & $\begin{array}{l}\text { Detailed domain configuration, including } \\
\text { E-values from Pfam analysis }\end{array}$ \\
\hline RC0G0129500 & $\mathrm{rc} 00$ & 15921725 & 15925651 & NBS-LRR & & P-loop[465-473] Kin-2[546-553] RNBS-B[573-576] GLPL[629-635] & $\begin{array}{l}\text { [440-721]NB-ARC:6.8e-40 [872- } \\
\text { 926]LRR_8:4.7e-07 } \\
\text { [467-747]NB-ARC:1.3e-38 [908- }\end{array}$ \\
\hline RC0G0129700 & $\mathrm{rc00}$ & 15936605 & 15940195 & NBS-LRR & & P-loop[491-499] Kin-2[572-579] RNBS-B[599-605] GLPL[661-667] & 964]LRR_8:9.6e-08 \\
\hline RC0G0157500 & $\mathrm{rc} 00$ & 20165214 & 20168978 & NBS & & P-loop[100-108] Kin-2[181-188] RNBS-B[209-215] GLPL[276-282] & $\begin{array}{l}\text { [76-370]NB-ARC:6.2e-73 } \\
{[4-135] \text { RPW8:8.3e-22 [165-435]NB- }}\end{array}$ \\
\hline RC0G0174400 & $\mathrm{rc00}$ & 22640609 & 22644651 & NBS & RPW8 & P-loop[186-194] Kin-2[267-274] RNBS-B[292-298] GLPL[347-353] & $\begin{array}{l}\text { ARC:1.7e-34 } \\
\text { [3-113]RPW8:7e-17 [165-429]NB- }\end{array}$ \\
\hline RC0G0176800 & $\mathrm{rc00}$ & 22873190 & 22878387 & NBS-LRR & RPW8 & P-loop[184-192] Kin-2[263-270] RNBS-B[288-294] GLPL[343-349] & $\begin{array}{l}\text { ARC:5.1e-34 [628-684]LRR_8:1.2e-05 } \\
\text { [724-763]LRR_4:0.00046 } \\
\text { [172-194]AAA_16:0.0003 [196-458]NB- }\end{array}$ \\
\hline RC0G0200900 & $\mathrm{rc00}$ & 26709295 & 26713250 & NBS-LRR & & P-loop[205-213] Kin-2[280-287] RNBS-B[310-316] GLPL[369-375] & $\begin{array}{l}\text { ARC:7.2e-61 [578-615]LRR_8:0.0026 } \\
\text { [620-656]LRR_8:0.0064 }\end{array}$ \\
\hline RC0G0201600 & $\mathrm{rc} 00$ & 26778236 & 26782002 & NBS-LRR & & P-loop[205-213] Kin-2[280-287] RNBS-B[310-316] GLPL[369-375] & $\begin{array}{l}\text { [172-194]AAA_16:1.9e-05 [196-455]NB- } \\
\text { ARC:2.2e-60 [576-613]LRR_8:0.0037 } \\
\text { [83-105]AAA_16:0.00016 [107-367]NB- }\end{array}$ \\
\hline RC0G0202800 & $\mathrm{rc} 00$ & 26907214 & 26909933 & NBS-LRR & & P-loop[116-124] Kin-2[191-198] RNBS-B[221-227] GLPL[280-286] & $\begin{array}{l}\text { 564]LRR_8:0.006 } \\
\text { [184-464]NB-ARC:1.4e-59 [574- }\end{array}$ \\
\hline RC0G0283700 & $\mathrm{rc} 00$ & 44426071 & 44428206 & CC-NBS-LRR & & P-loop[205-213] Kin-2[282-289] RNBS-B[313-319] GLPL[376-382] & $\begin{array}{l}\text { 623]LRR_8:2e-07 [630-652]LRR_4:8.3e- } \\
05\end{array}$ \\
\hline RC1G0009500 & $\mathrm{rc01}$ & 1503964 & 1506714 & NBS & & P-loop[26-34] Kin-2[104-111] RNBS-B[132-138] GLPL[191-197] & [2-263]NB-ARC:6.7e-37 \\
\hline RC1G0012400 & $\mathrm{rc01}$ & 1742358 & 1745854 & CC-NBS-LRR & & P-loop[201-209] Kin-2[277-284] RNBS-B[307-313] GLPL[368-374] & $\begin{array}{l}\text { [170-191]AAA_16:0.00058 [192-453]NB- } \\
\text { ARC:9.3e-64 [583-639]LRR_8:0.00043 }\end{array}$ \\
\hline RC1G0015400 & $\mathrm{rc} 01$ & 1977225 & 1978112 & NBS & & P-loop[43-51] Kin-2[119-126] RNBS-B[149-155] GLPL[210-216] & [21-294]NB-ARC:2.3e-66 \\
\hline RC1G0035900 & $\mathrm{rc01}$ & 4772090 & 4776326 & NBS-LRR & & $\begin{array}{l}\text { P-loop[204-212] Kin-2[279-286] RNBS-B[310-316] GLPL[371-377] } \\
\text { P-loop[200-208] Kin-2[274-281] RNBS-B[305-311] GLPL[366-372] }\end{array}$ & $\begin{array}{l}\text { [173-182]AAA_16:3.2e-05 [183-461]NB- } \\
\text { ARC:2.5e-60 [877-933]LRR_8:0.00012 } \\
\text { [950-1009]LRR_8:0.0013 [1009- } \\
\text { 1038]LRR_4:0.0023 } \\
\text { [174-420]NB-ARC:1.2e-48 [934- } \\
\text { 975]LRR_4:0.00053 }\end{array}$ \\
\hline RC1G0037000 & $\mathrm{rc01}$ & 4845477 & 4854130 & NBS-LRR & & P-loop[202-210] Kin-2[277-284] RNBS-B[308-314] GLPL[369-375] & $\begin{array}{l}\text { [171-180]AAA_16:0.0022 [181-458]NB- } \\
\text { ARC:3e-60 [877-922]LRR_8:0.00088 [961- } \\
\text { 1002]LRR_4:0.0015 [1009- } \\
\text { 1048]LRR_4:0.0053 } \\
\text { [168-456]NB-ARC:3e-58 [584- }\end{array}$ \\
\hline RC1G0038100 & $\mathrm{rc} 01$ & 4945293 & 4950173 & NBS-LRR & & P-loop[197-205] Kin-2[271-278] RNBS-B[304-310] GLPL[366-372] & $\begin{array}{l}\text { 638]LRR_8:9e-06 } \\
\text { [18-189]TIR:9.3e-41 [206-459]NB- }\end{array}$ \\
\hline RC1G0038200 & $\mathrm{rc01}$ & 4997244 & 5004392 & TIR-NBS-LRR & & P-loop[227-235] Kin-2[301-308] RNBS-B[332-338] GLPL[390-396] & $\begin{array}{l}\text { ARC:2.1e-34 [610-628]LRR_3:0.00055 } \\
\text { [704-761]LRR_8:0.00073 }\end{array}$ \\
\hline RC1G0041300 & $\mathrm{rc01}$ & 5325468 & 5330421 & NBS-LRR & & P-loop[205-213] Kin-2[280-287] RNBS-B[309-315] GLPL[370-376] & $\begin{array}{l}\text { [172-193]AAA_16:0.0076 [194-455]NB- } \\
\text { ARC:5.5e-66 [583-635]LRR_8:3.8e-05 } \\
\text { [636-679]LRR_8:0.00045 [680- } \\
\text { 698]LRR_4:0.0018 } \\
\text { [180-455]NB-ARC:9.8e-56 [583- } \\
\text { 637]LRR_8:7.1e-05 [637- }\end{array}$ \\
\hline RC1G0046400 & $\mathrm{rc} 01$ & 6001575 & 6004952 & NBS-LRR & & P-loop[205-213] Kin-2[279-286] RNBS-B[308-314] GLPL[369-375] & 666]LRR_4:0.009 \\
\hline RC1G0046600 & $\mathrm{rc} 01$ & 6015473 & 6021617 & CC-NBS-LRR & & P-loop[204-212] Kin-2[288-295] RNBS-B[317-323] GLPL[378-384] & [177-468]NB-ARC:1.1e-63 \\
\hline
\end{tabular}




\begin{tabular}{|c|c|c|c|c|c|c|c|}
\hline RC1G0046700 & $\mathrm{rc} 01$ & 6022946 & 6027291 & NBS-LRR & & P-loop[206-214] Kin-2[281-288] RNBS-B[310-316] GLPL[371-377] & $\begin{array}{l}\text { 621]LRR_4:0.0016 [622- } \\
\text { 635]LRR_4:0.0022 }\end{array}$ \\
\hline RC1G0054000 & $\mathrm{rc01}$ & 6873096 & 6882290 & NBS-LRR & & P-loop[205-213] Kin-2[280-287] RNBS-B[310-316] GLPL[369-375] & $\begin{array}{l}\text { [172-194]AAA_16:8.8e-05 [196-457]NB- } \\
\text { ARC:4.1e-63 [579-616]LRR_4:0.0073 } \\
\text { [172-194]AAA_16:2.2e-05 [196-457]NB- }\end{array}$ \\
\hline RC1G0054700 & $\mathrm{rc} 01$ & 6918760 & 6921635 & NBS-LRR & & P-loop[205-213] Kin-2[281-288] RNBS-B[311-317] GLPL[370-376] & $\begin{array}{l}\text { ARC:3.5e-63 [580-634]LRR_8:4.1e-05 } \\
\text { [634-654]LRR_8:0.00033 }\end{array}$ \\
\hline RC1G0055000 & $\mathrm{rc01}$ & 7020069 & 7022309 & NBS-LRR & & P-loop[205-213] Kin-2[280-287] RNBS-B[310-316] GLPL[369-375] & $\begin{array}{l}\text { [172-194]AAA_16:7.2e-06 [195-457]NB- } \\
\text { ARC:5.7e-62 [579-633]LRR_8:8.9e-06 }\end{array}$ \\
\hline RC1G0057100 & $\mathrm{rc} 01$ & 7250515 & 7253199 & CC-NBS-LRR & & P-loop[199-207] Kin-2[273-280] RNBS-B[303-309] GLPL[361-367] & $\begin{array}{l}\text { [166-189]AAA_16:3.7e-06 [190-449]NB- } \\
\text { ARC:6.8e-59 [563-572]LRR_8:0.0017 } \\
\text { [573-594]LRR_4:0.00075 [595- } \\
\text { 647]LRR_8:8.5e-05 }\end{array}$ \\
\hline RC1G0057400 & $\mathrm{rc} 01$ & 7348754 & 7351644 & NBS-LRR & & P-loop[203-211] Kin-2[278-285] RNBS-B[308-314] GLPL[367-373] & $\begin{array}{l}\text { [170-192]AAA_16:1.3e-05 [194-454]NB- } \\
\text { ARC:5.6e-63 [572-609]LRR_8:0.0065 } \\
\text { [73-95]AAA_16:5.5e-05 [96-356]NB- } \\
\text { ARC:1.9e-59 [477-500]LRR_8:0.00043 }\end{array}$ \\
\hline RC1G0057600 & $\mathrm{rc} 01$ & 7392967 & 7394847 & NBS-LRR & & P-loop[106-114] Kin-2[180-187] RNBS-B[210-216] GLPL[268-274] & $\begin{array}{l}\text { [501-541]LRR_4:4.9e-05 } \\
\text { [168-190]AAA_16:0.00032 [192-455]NB- }\end{array}$ \\
\hline RC1G0058500 & $\mathrm{rc} 01$ & 7430559 & 7433431 & NBS-LRR & & P-loop[201-209] Kin-2[276-283] RNBS-B[306-312] GLPL[369-375] & $\begin{array}{l}\text { ARC:1.1e-63 } \\
\text { [172-194]AAA_16:8.7e-05 [196-456]NB- } \\
\text { ARC:2e-61 [578-632]LRR_8:7.3e-05 [632- } \\
\text { 652]LRR_8:0.0069 }\end{array}$ \\
\hline RC1G0061100 & $\mathrm{rc01}$ & 7727136 & 7629494 & CC-NBS-LRR & & P-loop[205-213] Kin-2[280-287] RNBS-B[310-316] GLPL[369-375] & $\begin{array}{l}\text { [172-194]AAA_16:1.4e-05 [196-456]NB- } \\
\text { ARC:2.9e-61 [579-633]LRR_8:7.2e-05 } \\
\text { [172-194]AAA_16:2.1e-05 [195-456]NB- } \\
\text { ARC:1.1e-63 [579-633]LRR_8:0.00012 } \\
\text { [640-653]LRR_8:0.0025 }\end{array}$ \\
\hline RC1G0064300 & $\mathrm{rc} 01$ & 8054123 & 8056050 & TIR-NBS & & P-loop[133-141] Kin-2[211-218] RNBS-B[238-244] GLPL[297-303] & $\begin{array}{l}\text { [3-95]TIR:2.4e-07 [111-121]AAA_16:3.9e- } \\
06 \text { [122-365]NB-ARC:1.4e-33 }\end{array}$ \\
\hline RC1G0065800 & $\mathrm{rc} 01$ & 8149862 & 8157268 & NBS-LRR & & P-loop[200-208] Kin-2[272-279] RNBS-B[302-308] GLPL[358-364] & $\begin{array}{l}\text { [168-190]AAA_16:3.1e-05 [191-446]NB- } \\
\text { ARC:7.1e-62 [570-608]LRR_4:3.9e-05 }\end{array}$ \\
\hline RC1G0069700 & $\mathrm{rc01}$ & 8703984 & 8706776 & NBS-LRR & & P-loop[27-35] Kin-2[105-112] RNBS-B[132-138] GLPL[191-197] & $\begin{array}{l}\text { [13-261]NB-ARC:2e-28 [409- } \\
\text { 427]LRR_3:1.8e-05 [477- } \\
\text { 500]LRR_4:0.00094 [501- } \\
\text { 557]LRR_8:0.0002 [557-581]LRR_8:0.004 }\end{array}$ \\
\hline RC1G0073500 & $\mathrm{rc} 01$ & 9041245 & 9043098 & TIR-NBS & & P-loop[127-135] Kin-2[205-212] RNBS-B[232-238] GLPL[291-297] & $\begin{array}{l}\text { [2-93]TIR:9.3e-10 [107-117]AAA_16:4.2e- } \\
\text { 06 [118-306]NB-ARC:1.2e-24 } \\
\text { [18-258]NB-ARC:5.9e-30 [409- }\end{array}$ \\
\hline RC1G0074800 & $\mathrm{rc} 01$ & 9109406 & 9111181 & NBS & & P-loop[27-35] Kin-2[105-112] RNBS-B[132-138] GLPL[191-197] & $\begin{array}{l}\text { 427]LRR_3:1.2e-05 } \\
{[14-284] \text { NB-ARC:4.7e-57 [386- }}\end{array}$ \\
\hline RC1G0102900 & $\mathrm{rc} 01$ & 13141019 & 13142561 & NBS-LRR & & P-loop[35-43] Kin-2[110-117] RNBS-B[139-145] GLPL[196-202] & 444]LRR_8:1.8e-07 \\
\hline RC1G0105100 & $\mathrm{rc} 01$ & 13684527 & 13694466 & CC-NBS-LRR & RPW8 & $\begin{array}{l}\text { P-loop[904-912] Kin-2[985-992] RNBS-B[1009-1015] GLPL[1063- } \\
\text { 1069] }\end{array}$ & $\begin{array}{l}\text { [2-134]RPW8:3.7e-28 [172-435]NB- } \\
\text { ARC:5e-35 [896-1145]NB-ARC:3.2e-27 }\end{array}$ \\
\hline RC1G0105300 & $\mathrm{rc} 01$ & 13704406 & 13708236 & CC-NBS-LRR & RPW8 & P-loop[218-226] Kin-2[302-309] RNBS-B[328-333] GLPL[382-388] & $\begin{array}{l}\text { [2-111]RPW8:2.7e-13 [197-469]NB- } \\
\text { ARC:3.1e-32 [732-788]LRR_8:0.0027 } \\
\text { [174-453]NB-ARC:1e-62 [578- }\end{array}$ \\
\hline RC1G0107900 & $\mathrm{rc01}$ & 14058276 & 14061495 & CC-NBS-LRR & & P-loop[199-207] Kin-2[274-281] RNBS-B[303-309] GLPL[364-370] & 632]LRR_8:0.00033 \\
\hline
\end{tabular}




\begin{tabular}{|c|c|c|c|c|}
\hline RC1G0110400 & $\mathrm{rc} 01$ & 14418616 & 14420309 & CC-NBS \\
\hline RC1G0116200 & $\mathrm{rc} 01$ & 15333635 & 15336468 & NBS-LRR \\
\hline RC1G0119600 & $\mathrm{rc} 01$ & 15888685 & 15896466 & $\begin{array}{l}\text { CC-TIR-NBS- } \\
\text { LRR }\end{array}$ \\
\hline RC1G0122900 & $\mathrm{rc} 01$ & 16293738 & 16299190 & NBS-LRR \\
\hline RC1G0123100 & $\mathrm{rc} 01$ & 16303980 & 16307600 & NBS-LRR \\
\hline RC1G0126400 & $\mathrm{rc} 01$ & 16721193 & 16724833 & TIR-NBS \\
\hline RC1G0138100 & $\mathrm{rc} 01$ & 18228736 & 18232954 & TIR-NBS-LRR \\
\hline RC1G0164900 & $\mathrm{rc} 01$ & 22907764 & 22914556 & TIR-NBS-LRR \\
\hline RC1G0173500 & $\mathrm{rc} 01$ & 23919112 & 23922623 & NBS-LRR \\
\hline RC1G0188800 & $\mathrm{rc} 01$ & 25163338 & 25166861 & CC-TIR-NBS \\
\hline RC1G0189200 & $\mathrm{rc} 01$ & 25203533 & 25207311 & NBS-LRR \\
\hline RC1G0202100 & $\mathrm{rc} 01$ & 27188454 & 27191737 & CC-NBS \\
\hline RC1G0212800 & $\mathrm{rc} 01$ & 28792198 & 28806550 & TIR-NBS-LRR \\
\hline RC1G0222900 & $\mathrm{rc} 01$ & 30353802 & 30356870 & CC-NBS \\
\hline RC1G0226800 & $\mathrm{rc} 01$ & 30851240 & 30853319 & NBS-LRR \\
\hline RC1G0227400 & $\mathrm{rc} 01$ & 30924069 & 30929646 & CC-NBS-LRR \\
\hline RC1G0228100 & $\mathrm{rc} 01$ & 31007694 & 31010755 & CC-NBS-LRR \\
\hline RC1G0243000 & $\mathrm{rc} 01$ & 33017453 & 33020902 & NBS-LRR \\
\hline RC1G0243600 & $\mathrm{rc} 01$ & 33043255 & 33047625 & NBS \\
\hline RC1G0253600 & $\mathrm{rc} 01$ & 34296194 & 34299052 & NBS \\
\hline RC1G0255300 & $\mathrm{rc} 01$ & 34455174 & 34458473 & NBS-LRR \\
\hline RC1G0256100 & $\mathrm{rc} 01$ & 34528453 & 34531707 & NBS \\
\hline RC1G0256400 & $\mathrm{rc} 01$ & 34538433 & 34541505 & NBS \\
\hline
\end{tabular}

P-loop[199-207] Kin-2[274-281] RNBS-B[304-310] GLPL[362-368]

P-loop[194-202] Kin-2[270-277] RNBS-B[300-306] GLPL[361-367]

P-loop[358-366] Kin-2[433-440] RNBS-B[462-468] GLPL[523-529]

P-loop[81-89] Kin-2[155-162] RNBS-B[182-188] GLPL[241-247]

P-loop[34-42] Kin-2[113-120] RNBS-B[140-146] GLPL[199-205]

P-loop[231-239] Kin-2[306-313] RNBS-B[333-339] GLPL[391-397]

P-loop[227-235] Kin-2[302-309] RNBS-B[331-337] GLPL[389-395]

MRP-

$\mathrm{S} 25, \mathrm{zf}$

RVT

P-loop[224-232] Kin-2[306-313] RNBS-B[332-338] GLPL[396-402]

P-loop[205-213] Kin-2[278-285] RNBS-B[308-314] GLPL[367-373]

P-loop[262-270] Kin-2[337-344] RNBS-B[366-372] GLPL[427-433]

P-loop[197-205] Kin-2[272-279] RNBS-B[301-307] GLPL[362-368]

P-loop[121-129] Kin-2[197-204] RNBS-B[224-230] GLPL[284-290]

P-loop[234-242] Kin-2[308-315] RNBS-B[340-346] GLPL[414-420] P-loop[186-194] Kin-2[267-274] RNBS-B[295-301] GLPL[359-365]

P-loop[26-34] Kin-2[104-111] RNBS-B[131-137] GLPL[190-196]

P-loop[210-218] Kin-2[285-292] RNBS-B[316-322] GLPL[378-384

P-loop[197-205] Kin-2[272-279] RNBS-B[301-307] GLPL[362-368] P-loop[198-206] Kin-2[279-286] RNBS-B[307-313] GLPL[372-378] P-loop[270-278] Kin-2[351-358] RNBS-B[379-385] GLPL[444-450] P-loop[53-61] Kin-2[134-141] RNBS-B[163-169] GLPL[228-234]

P-loop[197-205] Kin-2[278-285] RNBS-B[306-312] GLPL[371-377] P-loop[197-205] Kin-2[278-285] RNBS-B[306-312] GLPL[371-377] P-loop[197-205] Kin-2[278-285] RNBS-B[306-312] GLPL[371-377]
[164-187]AAA_16:0.00012 [190-447]NBARC:2.5e-61

[171-446]NB-ARC:4.6e-63 [572-

627]LRR_8:3.4e-05

[23-193]TIR:1.8e-43 [332-612]NB-

ARC:5.3e-66 [734-789]LRR 8:0.0025

[60-312]NB-ARC:7.8e-35 [501-

540]LRR_4:0.0012 [541-

559]LRR_4:0.0013 [605-

646]LRR_4:0.00058

[17-273]NB-ARC:3.1e-35 [606-

662]LRR_8:0.00016

[30-192]TIR:2.5e-44 [210-418]NB-

ARC:9.7e-28

[22-187]TIR:2.2e-43 [206-477]NB-

ARC:1.4e-52 [576-634]LRR_8:6.1e-06 [16-189]TIR:1.1e-46 [217-464]NB-

ARC:6.7e-17 [584-632]MRP-S25:0.0048

[775-833]LRR_8:2.6e-05 [833-

847]LRR_8:0.00033 [848-

904]LRR 8:4.3e-06 [904-

927]LRR_8:0.00037 [942-

997]LRR_8:0.00036 [1264-1336]zf-

RVT:3.6e-16

[172-194]AAA 16:0.00016 [196-454]NBARC:9.8e-63 [598-638]LRR_4:0.0077

[2-89]TIR:1.2e-10 [235-517]NB-ARC:2.5e-

[170-451]NB-ARC:9.7e-66 [589643]LRR_8:0.00012 [794-

825]LRR_4:0.0019

[103-370]NB-ARC:3.3e-70

[24-192]TIR:1.1e-43 [222-483]NBARC:2.8e-22 [634-651]LRR_3:4.2e-05

[730-788]LRR 8:0.00017 [905-

939]LRR 8:0.0056

[164-450]NB-ARC:2e-65

[4-256]NB-ARC:2.5e-35 [408-

463]LRR 8:0.001 [477-516]LRR 4:0.0044

[179-200]AAA_16:0.0038 [202-445]NB-

ARC:1.4e-48 [524-548]LRR_8:7.3e-07

[549-602]LRR_8:6.6e-07

[171-451]NB-ARC:2.2e-65 [576-

630]LRR_8:0.0063

[178-472]NB-ARC:7.6e-71

[250-544]NB-ARC:1.3e-71

[29-314]NB-ARC:6.1e-64

[174-471]NB-ARC:4.5e-67 [583-

624]LRR_8:0.0071

[175-472]NB-ARC:8e-74

[173-471]NB-ARC:1.3e-66 


\begin{tabular}{|c|c|c|c|c|}
\hline RC1G0257500 & $\mathrm{rc} 01$ & 34582155 & 34588844 & NBS \\
\hline RC1G0258100 & $\mathrm{rc} 01$ & 34682821 & 34686034 & NBS \\
\hline RC1G0258500 & $\mathrm{rc} 01$ & 34709625 & 34713095 & NBS-LRR \\
\hline RC1G0260000 & $\mathrm{rc} 01$ & 34855716 & 34858966 & CC-NBS-LRR \\
\hline RC1G0260300 & $\mathrm{rc} 01$ & 34910010 & 34911008 & NBS \\
\hline RC1G0260800 & $\mathrm{rc} 01$ & 34935325 & 34938804 & CC-NBS-LRR \\
\hline RC1G0261200 & $\mathrm{rc} 01$ & 34977136 & 34979891 & NBS-LRR \\
\hline RC1G0261500 & $\mathrm{rc} 01$ & 34992791 & 34995628 & CC-NBS-LRR \\
\hline RC1G0263800 & $\mathrm{rc} 01$ & 35482052 & 35484165 & NBS-LRR \\
\hline RC1G0266700 & $\mathrm{rc01}$ & 35983205 & 35987660 & TIR-NBS-LRR \\
\hline RC1G0267700 & $\mathrm{rc} 01$ & 36085877 & 36095663 & NBS-LRR \\
\hline RC1G0274300 & $\mathrm{rc} 01$ & 37005938 & 37008680 & NBS-LRR \\
\hline RC1G0274700 & $\mathrm{rc} 01$ & 37056770 & 37059016 & NBS-LRR \\
\hline RC1G0276600 & $\mathrm{rc} 01$ & 37308983 & 37312698 & $\begin{array}{l}\text { CC-TIR-NBS- } \\
\text { LRR }\end{array}$ \\
\hline RC1G0277100 & $\mathrm{rc} 01$ & 37347001 & 37349814 & NBS-LRR \\
\hline RC1G0279800 & $\mathrm{rc} 01$ & 37721000 & 37724162 & TIR-NBS-LRR \\
\hline RC1G0280200 & $\mathrm{rc} 01$ & 37745457 & 37750723 & TIR-NBS-LRR \\
\hline RC1G0280400 & $\mathrm{rc} 01$ & 37798453 & 37800765 & TIR-NBS-LRR \\
\hline RC1G0280700 & $\mathrm{rc} 01$ & 37844788 & 37848991 & TIR-NBS-LRR \\
\hline RC1G0280900 & $\mathrm{rc} 01$ & 37882803 & 37884682 & TIR-NBS \\
\hline RC1G0281100 & $\mathrm{rc} 01$ & 37889095 & 37900487 & NBS-LRR \\
\hline RC1G0281700 & $\mathrm{rc} 01$ & 37955383 & 37958948 & CC-NBS-LRR \\
\hline RC1G0282300 & $\mathrm{rc} 01$ & 38053236 & 38056766 & CC-NBS-LRR \\
\hline
\end{tabular}

P-loop[200-208] Kin-2[281-288] RNBS-B[309-315] GLPL[374-380] [177-475]NB-ARC:5.7e-70

P-loop[168-176] Kin-2[249-256] RNBS-B[277-283] GLPL[342-348] [146-443]NB-ARC:2.4e-70

[175-470]NB-ARC:1e-70 [803-

P-loop[198-206] Kin-2[279-286] RNBS-B[307-313] GLPL[369-375] 834]LRR_4:0.0045

[173-453]NB-ARC:1.5e-61 [573-

P-loop[198-206] Kin-2[273-280] RNBS-B[302-308] GLPL[363-369] 624]LRR_8:5.5e-05

P-loop[63-71] Kin-2[138-145] RNBS-B[167-173] GLPL[228-234] [40-305]NB-ARC:1.8e-63

[234-516]NB-ARC:9.1e-64 [658-

P-loop[260-268] Kin-2[335-342] RNBS-B[364-370] GLPL[425-431] 698]LRR_8:2.8e-05

[84-360]NB-ARC:9.7e-65 [476-

530]LRR_8:9.5e-07 [530-

P-loop[106-114] Kin-2[181-188] RNBS-B[210-216] GLPL[271-277] 553]LRR_4:0.0097

[171-452]NB-ARC:6.2e-65 [570-

624]LRR_8:2.9e-05 [624-

P-loop[197-205] Kin-2[272-279] RNBS-B[301-307] GLPL[362-368] 653]LRR_4:0.00081

$\begin{array}{ll}\text { P-loop[1-8] Kin-2[75-82] RNBS-B[104-110] GLPL[165-171] } & \text { [1-254]NB-ARC:1.3e-61 [376- } \\ & \text { 430]LRR_8:1.9e-06 } \\ & \text { [17-180]TIR:1.4e-45 [197-447]NB- } \\ & \text { ARC:1.8e-30 [611-666]LRR_8:0.00052 }\end{array}$

P-loop[219-227] Kin-2[298-305] RNBS-B[325-331] GLPL[384-390] [769-808]LRR_8:0.00026

[1-227]NB-ARC:1.1e-28 [541-

597]LRR 8:2.6e-05

[134-414]NB-ARC:8e-65 [537-

575]LRR_4:0.00013 [576

P-loop[157-165] Kin-2[232-239] RNBS-B[261-267] GLPL[324-330] 594]LRR_8:0.00095

[41-319]NB-ARC:1.4e-57 [439

P-loop[64-72] Kin-2[139-146] RNBS-B[168-174] GLPL[229-235]

494]LRR_8:3.6e-05

[23-193]TIR:5.6e-46 [329

350]AAA 16:0.0018 [351-617]NB-

ARC:3.9e-68 [739-752]LRR 4:0.0075

P-loop[360-368] Kin-2[435-442] RNBS-B[464-470] GLPL[525-531] $\quad \begin{aligned} & \text { ARC:353-795]LRR_8:0.0023 } \\ & \text { [171-462]NB-AR:2.5e-87 }\end{aligned}$

[171-462]NB-ARC:2.5e-87 [580-

636]LRR_8:0.0023 [848-

P-loop[194-202] Kin-2[278-285] RNBS-B[305-311] GLPL[369-375]

888]LRR_4:0.0039

[23-188]TIR:6.8e-47 [204-457]NB-

ARC:3e-30 [621-676]LRR 8:7.5e-05 [677-

$\begin{array}{ll}\text { P-loop[228-236] Kin-2[307-314] RNBS-B[334-340] GLPL[393-399] } & \text { 724]LRR_8:0.00036 } \\ & \text { [24-188]TIR:4.1e-44 [207-456]NB- }\end{array}$

[24-188]TIR:4.1e-44 [207-456]NB-

P-loop[228-236] Kin-2[307-314] RNBS-B[334-340] GLPL[393-399] [779-830]LRR_8:0.00025

[2-87]TIR:1.9e-15 [104-356]NB-ARC:1.6e-

P-loop[128-136] Kin-2[207-214] RNBS-B[234-240] GLPL[293-299] 33 [520-576]LRR_8:8.1e-05

[22-183]TIR:8.7e-47 [202-456]NB-

P-loop[226-234] Kin-2[305-312] RNBS-B[332-338] GLPL[391-397] ARC:1.9e-33 [610-628]LRR_3:7.8e-05

[22-183]TIR:1.3e-45 [202-457]NB-

P-loop[226-234] Kin-2[305-312] RNBS-B[332-338] GLPL[391-397] ARC:5.3e-34

[43-295]NB-ARC:3.7e-30 [611-

P-loop[65-73] Kin-2[144-151] RNBS-B[171-177] GLPL[230-236]

666]LRR_8:2.2e-07

[154-435]NB-ARC:3.6e-62 [554-

609]LRR_8:1.4e-05 [610-

638]LRR 4:0.00029 [971

P-loop[180-188] Kin-2[255-262] RNBS-B[284-290] GLPL[346-352] 1024]LRR_8:0.0023

[156-434]NB-ARC:2.2e-62 [554-

P-loop[180-188] Kin-2[255-262] RNBS-B[284-290] GLPL[346-352] 609]LRR_8:1.5e-05 [610- 
$38244919 \quad 38248918 \quad$ NBS-LRR

\begin{tabular}{|c|c|c|c|c|}
\hline RC1G0288000 & $\mathrm{rc} 01$ & 38848444 & 38859425 & TIR-NBS-LRR \\
\hline RC1G0288100 & $\mathrm{rc} 01$ & 38900986 & 38908393 & NBS-LRR \\
\hline RC1G0292300 & $\mathrm{rc} 01$ & 39326948 & 39330621 & CC-NBS-LRR \\
\hline RC1G0300200 & $\mathrm{rc} 01$ & 40223859 & 40231796 & NBS-LRR \\
\hline RC1G0329000 & $\mathrm{rc} 01$ & 43472577 & 43474189 & TIR-NBS \\
\hline RC1G0358000 & $\mathrm{rc} 01$ & 45960816 & 45969304 & NBS \\
\hline RC1G0363100 & $\mathrm{rc} 01$ & 46493639 & 46504568 & NBS-LRR \\
\hline RC1G0367100 & $\mathrm{rc} 01$ & 46770348 & 46773547 & NBS-LRR \\
\hline RC1G0400700 & $\mathrm{rc} 01$ & 50256161 & 50257511 & CC-NBS \\
\hline RC1G0404100 & $\mathrm{rc} 01$ & 50508504 & 50510713 & NBS-LRR \\
\hline RC1G0435000 & $\mathrm{rc} 01$ & 53058281 & 53067880 & TIR-NBS-LRR \\
\hline RC1G0441100 & $\mathrm{rc} 01$ & 53471786 & 53475028 & CC-NBS-LRR \\
\hline RC1G0454600 & $\mathrm{rc} 01$ & 54629504 & 54632323 & CC-NBS-LRR \\
\hline RC1G0454800 & $\mathrm{rc} 01$ & 54650760 & 54654953 & CC-NBS-LRR \\
\hline RC1G0461500 & $\mathrm{rc} 01$ & 55237811 & 55240714 & CC-NBS-LRR \\
\hline RC1G0497200 & $\mathrm{rc} 01$ & 57900419 & 57903163 & NBS-LRR \\
\hline RC1G0497800 & $\mathrm{rc} 01$ & 57955390 & 57958437 & NBS-LRR \\
\hline RC1G0510300 & $\mathrm{rc} 01$ & 58639792 & 58643017 & NBS-LRR \\
\hline
\end{tabular}

P-loop[65-73] Kin-2[144-151] RNBS-B[171-177] GLPL[230-236]

P-loop[218-226] Kin-2[296-303] RNBS-B[323-329] GLPL[379-385]

P-loop[218-226] Kin-2[296-303] RNBS-B[323-329] GLPL[379-385]

P-loop[26-34] Kin-2[103-110] RNBS-B[130-136] GLPL[186-192]

P-loop[193-201] Kin-2[268-275] RNBS-B[297-303] GLPL[359-365]

P-loop[202-210] Kin-2[277-284] RNBS-B[308-314] GLPL[369-375]

P-loop[225-233] Kin-2[300-307] RNBS-B[329-335] GLPL[387-393]

P-loop[171-179] Kin-2[245-252] RNBS-B[269-275] GLPL[328-334]

P-loop[194-202] Kin-2[275-282] RNBS-B[303-309] GLPL[368-374]

P-loop[194-202] Kin-2[275-282] RNBS-B[303-309] GLPL[370-376

P-loop[188-196] Kin-2[265-272] RNBS-B[294-300] GLPL[355-361]

P-loop[1-8] Kin-2[76-83] RNBS-B[103-109] GLPL[162-163]

P-loop[224-232] Kin-2[283-290] RNBS-B[310-316] GLPL[369-375]

P-loop[208-216] Kin-2[285-292] RNBS-B[314-320] GLPL[375-381]

P-loop[206-214] Kin-2[283-290] RNBS-B[312-318] GLPL[373-379]

P-loop[206-214] Kin-2[283-290] RNBS-B[312-318] GLPL[373-379]

P-loop[212-220] Kin-2[289-296] RNBS-B[318-324] GLPL[380-386

P-loop[26-34] Kin-2[104-111] RNBS-B[131-137] GLPL[188-194]

P-loop[26-34] Kin-2[104-111] RNBS-B[131-137] GLPL[188-194]

P-loop[199-207] Kin-2[274-281] RNBS-B[304-310] GLPL[362-368]
1-298]NB-ARC:1.6e-30 [459-

478]LRR_3:0.0042 [616-

670]LRR_8:0.00028

[14-192]TIR:1.1e-47 [193-

207]AAA_16:0.0028 [208-446]NB-

ARC:3.6e-24 [613-628]LRR_3:0.00089

[654-711]LRR_8:0.0042 [931-

[5-257]NB-ARC:1.4e-34 [557-

579]LRR_4:0.0099 [580-

579]LRR_4:0.0099 [580-
636]LRR_8:0.0078 [651-

689]LRR_4:0.0045 [821-875]LRR_8:7e-06

[182-449]NB-ARC:2.2e-66 [573-

[190-458]NB-ARC:6.1e-55 [838-

898]LRR 8:0.00016 [1009-

1134]LRR 5:4.9e-05

[20-182]TIR:2.6e-43 [204-412]NB-

ARC:6.6e-40

[151-415]NB-ARC: $1.3 \mathrm{e}-43$

[174-467]NB-ARC:7.2e-69 [621-

673]LRR_8:0.00042

[174-470]NB-ARC:2.8e-69 [767-

822]LRR_8:0.0085

[156-174]AAA_16:0.0039 [175-435]NB-

ARC:5e-63

[1-229]NB-ARC:1.4e-23

[18-198]TIR:2.1e-47 [203-435]NB-

ARC:2e-30 [587-642]LRR 8:0.0055

[176-188]AAA_16:0.0056 [189-463]NB-

ARC:4.3e-65 [587-642]LRR_8:3.2e-05

[174-184]AAA_16:0.00039 [186-459]NB ARC:3.9e-64 [597-645]LRR_8:0.00017

[174-186]AAA_16:6.2e-05 [187-461]NB-

ARC:6.8e-65 [560-578]LRR_4:0.002 [587-

627]LRR_4:0.00024

[180-198]AAA_16:0.00011 [199-467]NB-

ARC:9.4e-64 [620-660]LRR 4:1.3e-05

[669-708]LRR_4:0.00022

[3-254]NB-ARC:2.8e-29 [430-

449]LRR_3:2e-05 [499-

540]LRR_4:0.00028 [673-

727]LRR_8:2.4e-05

[3-255]NB-ARC:2.3e-30 [430-

449]LRR 3:3e-07 [674-728]LRR 8:1.7e06

[164-187]AAA 16:1.8e-05 [190-451]NB ARC:1.5e-61 [566-576]LRR 8:0.00018

[577-616]LRR 4:3.8e-05 [617-

651]LRR_8:6.2e-05 


$\begin{array}{lllll}\text { RC1G0519100 } & \text { rc01 } & 59266796 & 59269332 & \text { NBS-LRR } \\ \text { RC1G0522300 } & \mathrm{rc01} & 59490811 & 59493714 & \text { NBS-LRR } \\ & & 59873875 & 59875920 & \text { TIR-NBS } \\ \text { RC1G0528100 } & \mathrm{rc01} & 59891985 & 59893200 & \text { NBS }\end{array}$

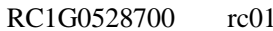

59911621

59918597

TIR-NBS-LRR WRKY

$59925434 \quad 59933238 \quad$ TIR-NBS-LRR

RC1G0528900 rc01

RC1G0579300 rc01

RC1G0579500 rc01

RC1G0581100 rc01

RC1G0581200 rc01

$63709713 \quad 63715148$ NBS-LRR

$\mathrm{RC} 2 \mathrm{G} 0007300 \quad \mathrm{rc} 02$

645051

661704 CC-NBS-LRR

RC2G0007700 rc02

RC2G0134700 rc02

$\mathrm{RC} 2 \mathrm{G} 0148100 \quad \mathrm{rc} 02$

678665 CC-NBS-LRR

11227056

11230506 TIR-NBS-LRR

12333147

12335759 NBS

$12581369 \quad 12586323 \quad$ NBS-LRR

RC2G0155400 rc02

$12912578 \quad 12916217 \quad$ CC-NBS-LRR

RC2G0179100 rc02

15325681

15329889 NBS-LRR

15342873

15347295 NBS-LRR

15385324

RC2G0179300 rc02

RC2G0179400 rc02
$15392613 \quad 15396430 \quad$ NBS-LRR
TniB

TniB

P-loop[199-207] Kin-2[274-281] RNBS-B[304-310] GLPL[362-368] P-loop[213-221] Kin-2[295-302] RNBS-B[322-328] GLPL[388-394]

P-loop[218-226] Kin-2[295-302] RNBS-B[322-328] GLPL[379-385]

P-loop[19-27] Kin-2[96-103] RNBS-B[123-129] GLPL[180-186]

P-loop[222-230] Kin-2[300-307] RNBS-B[327-333] GLPL[384-390]

P-loop[1131-1139] Kin-2[1209-1216] RNBS-B[1236-1242] GLPL[1291-1292]

P-loop[124-132] Kin-2[202-209] RNBS-B[229-235] GLPL[288-294]

P-loop[124-132] Kin-2[203-210] RNBS-B[230-236] GLPL[289-295]

P-loop[224-232] Kin-2[302-309] RNBS-B[329-335] GLPL[388-394] P-loop[26-34] Kin-2[105-112] RNBS-B[132-138] GLPL[191-197]

P-loop[176-184] Kin-2[253-260] RNBS-B[277-283] GLPL[337-343]

P-loop[185-193] Kin-2[265-272] RNBS-B[289-295] GLPL[347-353]

P-loop[226-234] Kin-2[300-307] RNBS-B[327-333] GLPL[386-392

RPW8

P-loop[208-216] Kin-2[282-289] RNBS-B[310-316] GLPL[374-380]

P-loop[135-143] Kin-2[212-219] RNBS-B[239-245] GLPL[300-306] P-loop[193-201] Kin-2[275-282] RNBS-B[302-308] GLPL[365-371]

P-loop[219-227] Kin-2[298-305] RNBS-B[324-330] GLPL[380-386]

RPW8

RPW8

RPW8

RPW8

P-loop[203-211] Kin-2[281-288] RNBS-B[307-313] GLPL[362-368]
[188-450]NB-ARC:2.2e-62 [556569]LRR_8:0.00024 [570-

624]LRR_8:1.1e-05 [624-

643]LRR_8:0.0068

[203-481]NB-ARC:7.5e-77 [601-

654]LRR_8:0.0052

[14-192]TIR:2.1e-47 [195-442]NB-

ARC: $1.3 \mathrm{e}-32$

[5-238]NB-ARC:9.3e-33

[18-183]TIR:3.6e-44 [203-451]NB-

ARC:1.6e-25 [618-637]LRR_3:4.4e-07

[687-724]LRR_4:0.00096 [725-

767]LRR_8:0.0075 [863-915]LRR_8:1.1e-

06 [915-925]LRR_4:0.0054 [1275-

1335]WRKY:1.3e-23

[18-197]TIR:1.1e-39 [201-446]NB-

ARC:2.9e-28 [618-637]LRR_3:0.00013

[1107-1316]NB-ARC:4.4e-18 [1511-

1530]LRR_3:3.7e-05 [1580-

1623]LRR_4:0.0019 [1629-

1684]LRR_8:0.00084

[4-98]TIR:2e-13 [104-357]NB-ARC:4e-38

[4-86]TIR:2.9e-12 [102-360]NB-ARC:3.2e-

37 [503-558]LRR_8:0.00041 [685-

727]LRR_4:0.0079

[18-197]TIR:3.9e-50 [206-455]NB-

ARC:2.9e-35 [604-659]LRR_8:0.0039

[4-254]NB-ARC:1.8e-38 [408-

463]LRR_8:0.00074

[157-424]NB-ARC:8.9e-42 [1272-

1282]TniB:0.0021 [1283-1554]NB-

ARC:1e-42 [1671-1715]LRR_8:0.0054

[1727-1784]LRR_8:0.0018

[156-166]TniB:0.0043 [167-426]NB-

ARC:1.8e-48 [608-663]LRR_8:0.0011

[20-200]TIR:5.5e-47 [211-454]NB-

ARC:7.4e-36

[23-98]RPW8:0.0034 [185-465]NB-

ARC:1.3e-89

[107-125]AAA_16:0.00053 [126-367]NB-

ARC:3.1e-29 [518-536]LRR_3:1.5e-05

[610-667]LRR_8:6.9e-06 [765-

822]LRR_8:0.0028

[170-457]NB-ARC:1.8e-78 [574-

630]LRR_8:1.6e-06 [631-

647]LRR_8:0.00012

[3-136]RPW8:6e-24 [199-467]NB-

ARC:9.4e-37

[3-136]RPW8:3.2e-30 [183-447]NB-

ARC: $1.9 e-25$ [710-766]LRR $8: 0.0032$

[21-157]RPW8:3.5e-31 [219-483]NB-

P-loop[239-247] Kin-2[318-325] RNBS-B[344-350] GLPL[396-402] ARC:7.5e-33

[1-132]RPW8:3.5e-26 [194-457]NB-

P-loop[214-222] Kin-2[293-300] RNBS-B[319-325] GLPL[371-377] ARC:1.5e-34 


\begin{tabular}{|c|c|c|c|c|c|c|c|}
\hline RC2G0179500 & $\mathrm{rc} 02$ & 15397426 & 15402277 & NBS & RPW8 & P-loop[215-223] Kin-2[294-301] RNBS-B[320-325] GLPL[374-380] & $\begin{array}{l}\text { [6-137]RPW8:1e-23 [201-462]NB- } \\
\text { ARC:2.9e-30 } \\
\text { 38-302]NB-ARC-5.6e-27 [540- }\end{array}$ \\
\hline RC2G0179600 & $\mathrm{rc} 02$ & 15402279 & 15405360 & NBS-LRR & & P-loop[54-62] Kin-2[134-141] RNBS-B[160-166] GLPL[215-221] & $\begin{array}{l}\text { 592]LRR_8:0.0028 } \\
\text { [5-140]RPW8:4.6e-28 [141- }\end{array}$ \\
\hline RC2G0179800 & $\mathrm{rc} 02$ & 15434947 & 15438315 & CC-NBS-LRR & $\begin{array}{l}\text { RPW8,Vps } \\
\text { 53_N }\end{array}$ & P-loop[231-239] Kin-2[310-317] RNBS-B[335-341] GLPL[389-395] & $\begin{array}{l}\text { 162]Vps53_N:0.0028 [211-475]NB- } \\
\text { ARC:3.4e-33 [741-798]LRR_8:5.6e-05 }\end{array}$ \\
\hline RC2G0180500 & $\mathrm{rc} 02$ & 15481969 & 15485440 & CC-NBS-LRR & RPW8 & P-loop[230-238] Kin-2[309-316] RNBS-B[334-340] GLPL[388-394] & $\begin{array}{l}\text { [5-140]RPW8:1e-26 [210-474]NB- } \\
\text { ARC:1.1e-31 [740-797]LRR_8:0.00019 } \\
\text { [6-140]RPW8:3e-27 [207-476]NB- }\end{array}$ \\
\hline RC2G0180800 & $\mathrm{rc} 02$ & 15510723 & 15513360 & CC-NBS & RPW8 & P-loop[227-235] Kin-2[306-313] RNBS-B[331-337] GLPL[390-396] & $\begin{array}{l}\text { ARC:2.7e-30 } \\
\text { [8-133]RPW8:8.6e-22 [191-458]NB- }\end{array}$ \\
\hline RC2G0181000 & $\mathrm{rc} 02$ & 15515501 & 15519519 & NBS & RPW8 & P-loop[213-221] Kin-2[290-297] RNBS-B[317-323] GLPL[372-378] & ARC: $7.2 \mathrm{e}-36$ \\
\hline RC2G0181200 & $\mathrm{rc} 02$ & 15537972 & 15543128 & NBS-LRR & RPW8 & P-loop[194-202] Kin-2[273-280] RNBS-B[299-305] GLPL[354-360] & $\begin{array}{l}\text { [5-139]RPW8:1.4e-24 [175-441]NB- } \\
\text { ARC:8.1e-35 [682-737]LRR_8:0.0017 } \\
\text { [1-103]RPW8:1.4e-17 [142-407]NB- }\end{array}$ \\
\hline RC2G0181300 & $\mathrm{rc} 02$ & 15551313 & 15554880 & CC-NBS & RPW8 & P-loop[161-169] Kin-2[240-247] RNBS-B[266-272] GLPL[321-327] & $\begin{array}{l}\text { ARC:1.1e-32 } \\
\text { [3-106]RPW8:3.9e-17 [149-412]NB- }\end{array}$ \\
\hline RC2G0181700 & $\mathrm{rc} 02$ & 15566602 & 15570265 & CC-NBS & RPW8 & P-loop[166-174] Kin-2[245-252] RNBS-B[271-277] GLPL[326-332] & ARC: $1.3 \mathrm{e}-31$ \\
\hline RC2G0183000 & $\mathrm{rc} 02$ & 15720908 & 15574784 & CC-NBS-LRR & $\begin{array}{l}\text { RPW8,Pkin } \\
\text { ase_Tyr }\end{array}$ & P-loop[417-425] Kin-2[481-488] RNBS-B[507-513] GLPL[562-568] & $\begin{array}{l}\text { [11-121]RPW8:1.8e-19 [173- } \\
\text { 187]AAA_16:0.00063 [188-447]NB- } \\
\text { ARC:1.6e-26 [699-746]LRR_8:0.0057 } \\
\text { [747-787]LRR_4:0.0016 } \\
\text { [5-138]RPW8:6.5e-13 [183- } \\
\text { 310]RPW8:1.9e-19 [399-648]NB- } \\
\text { ARC:4.3e-25 [887-945]LRR_8:0.0039 } \\
\text { [1167-1476]Pkinase_Tyr:4.4e-35 [1558- } \\
\text { 1837]Pkinase_Tyr:1.2e-45 }\end{array}$ \\
\hline $\mathrm{RC} 2 \mathrm{G} 0184000$ & $\mathrm{rc} 02$ & 15816412 & 15826862 & CC-NBS-LRR & $\begin{array}{l}\text { RPW8,zf- } \\
\mathrm{CCCH}\end{array}$ & P-loop[519-527] Kin-2[598-605] RNBS-B[623-629] GLPL[678-684] & $\begin{array}{l}\text { [12-144]RPW8:3.2e-12 [211- } \\
\text { 328]RPW8:6.9e-22 [439-463]zf- } \\
\text { CCCH:2.9e-08 [498-763]NB-ARC:2.3e-41 } \\
\text { [1015-1072]LRR_8:7.1e-05 } \\
\text { [6-140]RPW8:2.1e-33 [176-442]NB- }\end{array}$ \\
\hline $\mathrm{RC} 2 \mathrm{G} 0184100$ & $\mathrm{rc} 02$ & 15828907 & 15835140 & NBS & $\begin{array}{l}\text { RPW8,Pept } \\
\text { idase_C48 }\end{array}$ & P-loop[196-204] Kin-2[275-282] RNBS-B[300-306] GLPL[355-361] & $\begin{array}{l}\text { ARC:1e-24 [1024-1231]Peptidase_C48:6e- } \\
30 \\
\text { [56-227]TIR:2.8e-48 [232- } \\
\text { 241]AAA_16:0.0094 [242-491]NB- } \\
\text { ARC:9.8e-33 [894-950]LRR_8:1.2e-05 } \\
\text { [1017-1054]LRR_4:3.1e-05 [1152- } \\
\text { 1204]LRR_8:0.00093 [1244-- } \\
\text { 1280]LRR_8:3.3e-07 [1281- } \\
\text { 1313]LRR_4:0.00036 [1314- }\end{array}$ \\
\hline RC2G0199900 & $\mathrm{rc} 02$ & 17674833 & 17684919 & TIR-NBS-LRR & & P-loop[260-268] Kin-2[334-341] RNBS-B[365-371] GLPL[426-432] & $\begin{array}{l}\text { 1324]LRR_8:0.0028 } \\
\text { [23-194]TIR:4.7e-47 [208-466]NB- } \\
\text { ARC:9.1e-33 [716-774]LRR_8:0.0053 } \\
\text { [985-1022]LRR_4:0.0007 [1120- } \\
\text { 1173]LRR_8:0.0011 [1190- } \\
\text { 1249]LRR_8:5.5e-09 [1250- } \\
\text { 1282]LRR_4:0.00017 [1283- } \\
\text { 1293]LRR_8:0.0023 }\end{array}$ \\
\hline RCZG0200100 & $\mathrm{reO} 2$ & $1 / / 03046$ & $1 / 710202$ & R-NBS-LRR & & P-loop $[22 /-235]$ Kin-2[301-308] RNBS-B[352-358] GLPL & 1293]LRR_8:0.0023 \\
\hline
\end{tabular}




\begin{tabular}{|c|c|c|c|c|}
\hline RC2G0200200 & $\mathrm{rc} 02$ & 17718777 & 17727816 & TIR-NBS-LRR \\
\hline RC2G0210900 & $\mathrm{rc} 02$ & 18878750 & 18883160 & NBS-LRR \\
\hline RC2G0219600 & $\mathrm{rc} 02$ & 19753419 & 19761438 & NBS-LRR \\
\hline RC2G0244600 & $\mathrm{rc} 02$ & 22875094 & 22883763 & NBS-LRR \\
\hline RC2G0255900 & $\mathrm{rc} 02$ & 24654280 & 24655778 & NBS \\
\hline RC2G0285900 & $\mathrm{rc} 02$ & 28438869 & 28441360 & CC-NBS-LRR \\
\hline RC2G0294800 & $\mathrm{rc} 02$ & 29720246 & 29724405 & CC-NBS-LRR \\
\hline RC2G0314400 & $\mathrm{rc} 02$ & 32812925 & 32817044 & NBS-LRR \\
\hline RC2G0318800 & $\mathrm{rc} 02$ & 33687657 & 33689879 & NBS-LRR \\
\hline RC2G0319100 & $\mathrm{rc} 02$ & 33716915 & 33720694 & CC-NBS-LRR \\
\hline RC2G0350400 & $\mathrm{rc} 02$ & 39412156 & 39414798 & CC-NBS-LRR \\
\hline RC2G0350700 & $\mathrm{rc} 02$ & 39441734 & 39444415 & NBS-LRR \\
\hline RC2G0356300 & $\mathrm{rc} 02$ & 39969343 & 39973621 & NBS-LRR \\
\hline $\mathrm{RC} 2 \mathrm{G} 0358600$ & $\mathrm{rc} 02$ & 40163047 & 40168495 & NBS-LRR \\
\hline RC2G0389900 & $\mathrm{rc} 02$ & 44543234 & 44548635 & CC-NBS-LRR \\
\hline RC2G0392700 & $\mathrm{rc} 02$ & 44882897 & 44887968 & CC-NBS-LRR \\
\hline RC2G0394600 & $\mathrm{rc} 02$ & 45176707 & 45181083 & NBS \\
\hline $\mathrm{RC} 2 \mathrm{G} 0400800$ & $\mathrm{rc} 02$ & 46012932 & 46015626 & CC-NBS-LRR \\
\hline $\mathrm{RC} 2 \mathrm{G} 0401500$ & $\mathrm{rc} 02$ & 46046182 & 46047530 & NBS-LRR \\
\hline
\end{tabular}

P-loop[227-235] Kin-2[305-312] RNBS-B[336-342] GLPL[395-401]

P-loop[202-210] Kin-2[277-284] RNBS-B[308-314] GLPL[369-375]

P-loop[86-94] Kin-2[164-171] RNBS-B[188-194] GLPL[247-253]

P-loop[173-181] Kin-2[250-257] RNBS-B[274-280] GLPL[333-339]

P-loop[178-186] Kin-2[260-267] RNBS-B[288-294] GLPL[343-349]

P-loop[206-214] Kin-2[281-288] RNBS-B[315-321] GLPL[376-382]

P-loop[270-278] Kin-2[346-353] RNBS-B[375-381] GLPL[437-443

P-loop[206-214] Kin-2[281-288] RNBS-B[315-321] GLPL[376-382

P-loop[54-62] Kin-2[131-138] RNBS-B[159-165] GLPL[215-221]

P-loop[549-557] Kin-2[629-636] RNBS-B[656-662] GLPL[716-722]

P-loop[206-214] Kin-2[281-288] RNBS-B[315-321] GLPL[376-382]

P-loop[206-214] Kin-2[281-288] RNBS-B[315-321] GLPL[376-382]

P-loop[206-214] Kin-2[281-288] RNBS-B[315-321] GLPL[376-382]

P-loop[206-214] Kin-2[281-288] RNBS-B[315-321] GLPL[376-382]

P-loop[208-216] Kin-2[283-290] RNBS-B[312-318] GLPL[375-381]

P-loop[208-216] Kin-2[283-290] RNBS-B[312-318] GLPL[375-381]

P-loop[198-206] Kin-2[275-282] RNBS-B[305-311] GLPL[366-372]

P-loop[208-216] Kin-2[283-290] RNBS-B[312-318] GLPL[375-381]

P-loop[1-8] Kin-2[75-82] RNBS-B[104-110] GLPL[167-173]
[23-194]TIR:7.8e-49 [205-467]NB-

ARC:5e-31 [632-647]LRR_3:0.0018 [891-

926]LRR_8:0.0065 [1011-

1048]LRR 4:0.00013 [1219-

1241]LRR_8:2.6e-06 [1242-

1300]LRR_8:6e-12 [1301-

1323]LRR_8:2.8e-10[1324-

1323]LRR_8:2.8e-10 [1324-

1392]LRR_8:1.7e-09 [1393-

1402]LRR_8:2.6e-09 [1403-

1448]LRR_8:6e-10 [1449-

1507]LRR_8:5.5e-11 [1511-

1552]LRR 8:1.6e-05

[177-459]NB-ARC:2.8e-63 [562-

572]LRR_8:0.0051 [573-

629]LRR_8:0.00097 [1120-

1159]LRR_4:0.0016

67-333]NB-ARC:5.3e-50 [499-

555]LRR_8:0.0016

[151-403]NB-ARC:8.4e-36 [591-

628]LRR_8:0.0044 [628

652]LRR_8:0.0072

[148-167]AAA_16:0.0089 [168-355]NB-

ARC: $4.8 \mathrm{e}-24$

[180-464]NB-ARC:5e-59 [584-

640]LRR 8:0.00054

[254-525]NB-ARC:2.2e-69 [630-

649]LRR_8:5.1e-07 [650-705]LRR_8:2.7e-

09

[180-463]NB-ARC:3.5e-57 [585-

639]LRR_8:0.0043

[30-306]NB-ARC:8.6e-43 [467-

522]LRR_8:5.4e-08

[524-803]NB-ARC:3.9e-42 [958-

1013]LRR 8:6.1e-05

[173-194]AAA_16:0.0035 [195-464]NB-

ARC:3.5e-56 [584-638]LRR_8:9.6e-06

[178-461]NB-ARC:2.8e-57 [617-

655]LRR_4:0.0011

[178-462]NB-ARC:4.9e-57

[195-462]NB-ARC:7.7e-56 [584-

605]LRR_8:0.0033 [606-

645]LRR_4:0.0013

[185-462]NB-ARC:5.4e-63 [562-

618]LRR_8:1.1e-08 [625-

641]LRR_8:0.00058

[185-464]NB-ARC:1.3e-64 [568-

590]LRR_8:7.4e-07 [591-647]LRR_8:1.6e-

07 [788-841]LRR_8:0.0055

[174-451]NB-ARC:1.3e-60

[184-464]NB-ARC:7.1e-62 [562-

584]LRR_8:1.2e-07 [585-641]LRR_8:7.5e-

08

[1-239]NB-ARC:9.4e-43 [347-

396]LRR_8:3.2e-07 


\begin{tabular}{|c|c|c|c|c|c|c|c|}
\hline RC2G0401600 & $\mathrm{rc02}$ & 46049909 & 46052803 & CC-NBS-LRR & & P-loop[208-216] Kin-2[283-290] RNBS-B[312-318] GLPL[375-381] & $\begin{array}{l}\text { 584]LRR_8:9.4e-07 [585-641]LRR_8:4.1e- } \\
08 \text { [780-833]LRR_8:0.0092 } \\
\text { [85-367]NB-ARC:4.7e-63 [490- }\end{array}$ \\
\hline RC2G0401900 & $\mathrm{rc02}$ & 46068087 & 46070552 & NBS-LRR & & P-loop[113-121] Kin-2[188-195] RNBS-B[217-223] GLPL[280-286] & $\begin{array}{l}\text { 544]LRR_8:1e-08 } \\
\text { [177-189]AAA_16:0.00095 [190-464]NB- }\end{array}$ \\
\hline RC2G0402100 & $\mathrm{rc02}$ & 46079724 & 46083075 & CC-NBS-LRR & & P-loop[208-216] Kin-2[283-290] RNBS-B[312-318] GLPL[375-381] & $\begin{array}{l}\text { ARC:1.8e-63 [562-584]LRR_8:1.5e-07 } \\
\text { [585-640]LRR_8:1.2e-07 } \\
\text { [186-465]NB-ARC:7.1e-62 [562- } \\
\text { 584]LRR 8:1.5e-07 [585-641]LRR 8:5.9e- }\end{array}$ \\
\hline RC2G0402400 & $\mathrm{rc} 02$ & 46117552 & 46120801 & CC-NBS-LRR & & P-loop[208-216] Kin-2[283-290] RNBS-B[312-318] GLPL[375-381] & 08 \\
\hline RC2G0403100 & $\mathrm{rc} 02$ & 46146324 & 46149074 & CC-NBS-LRR & & P-loop[208-216] Kin-2[284-291] RNBS-B[313-319] GLPL[376-382] & $\begin{array}{l}\text { [185-463]NB-ARC:7.5e-62 } \\
\text { [1-255]NB-ARC:1.3e-56 [354- }\end{array}$ \\
\hline RC2G0422900 & $\mathrm{rc02}$ & 49299023 & 49302923 & CC-NBS-LRR & CSD & P-loop[215-223] Kin-2[292-299] RNBS-B[321-327] GLPL[384-390] & $\begin{array}{l}\text { 376]LRR_8:2.9e-07 [377-433]LRR_8:1e- } \\
07 \\
\text { [186-472]NB-ARC:7.2e-62 [567- } \\
\text { 623]LRR_8:2e-07 [631- } \\
\text { 654]LRR_4:0.00062 [1004- } \\
\text { 1069]CSD:7.6e-24 }\end{array}$ \\
\hline RC2G0424700 & $\mathrm{rc02}$ & 49551738 & 49556053 & CC-NBS & & P-loop[208-216] Kin-2[284-291] RNBS-B[313-319] GLPL[375-381] & $\begin{array}{l}{[191-463] N B-A R C: 8.4 \mathrm{e}-70} \\
\text { [179-455]NB-ARC:1.7e-63 [564- } \\
\text { 580]LRR } 8 \cdot 9-5 \mathrm{e}-06 \text { [581-633]LRR } 8 \cdot 3.2 \mathrm{e}-\end{array}$ \\
\hline RC2G0425100 & $\mathrm{rc} 02$ & 49561390 & 49564576 & CC-NBS-LRR & & P-loop[200-208] Kin-2[276-283] RNBS-B[305-311] GLPL[368-374] & $\begin{array}{l}07 \text { [634-659]LRR_8:0.0019 } \\
\text { [171-181]AAA_16:0.0028 [182-386]NB- }\end{array}$ \\
\hline RC2G0428200 & $\mathrm{rc02}$ & 49894772 & 49895982 & CC-NBS & & P-loop[203-211] Kin-2[279-286] RNBS-B[308-314] GLPL[371-377] & $\begin{array}{l}\text { ARC:7e-45 } \\
\text { [192-466]NB-ARC:3.4e-71 [587- } \\
\text { 642]LRR_8:1.4e-08 [657- }\end{array}$ \\
\hline RC2G0428600 & $\mathrm{rc02}$ & 49927478 & 49934703 & CC-NBS-LRR & & P-loop[209-217] Kin-2[285-292] RNBS-B[314-320] GLPL[376-382] & 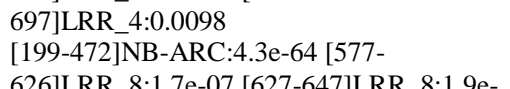 \\
\hline RC2G0428800 & $\mathrm{rc02}$ & 49938166 & 49940232 & CC-NBS-LRR & & P-loop[216-224] Kin-2[292-299] RNBS-B[322-328] GLPL[384-390] & $\begin{array}{l}\text { 626]LRR_8:1.7e-07 [627-647]LRR_8:1.9e- } \\
07 \text { [656-671]LRR_8:0.0036 } \\
\text { [194-465]NB-ARC:2.6e-69 [588- }\end{array}$ \\
\hline RC2G0429900 & $\mathrm{rc02}$ & 50004991 & 50007661 & CC-NBS-LRR & & P-loop[210-218] Kin-2[286-293] RNBS-B[315-321] GLPL[377-383] & $\begin{array}{l}\text { 643]LRR_8:6.7e-08 } \\
\text { [194-465]NB-ARC:3.5e-69 [588- }\end{array}$ \\
\hline RC2G0430300 & $\mathrm{rc02}$ & 50021196 & 50024246 & CC-NBS-LRR & & P-loop[210-218] Kin-2[286-293] RNBS-B[315-321] GLPL[377-383] & $\begin{array}{l}\text { 643]LRR_8:8e-08 } \\
\text { [176-463]NB-ARC:9.6e-83 [557- } \\
\text { 608]LRR 8:0.00031 [609- }\end{array}$ \\
\hline RC2G0431500 & $\mathrm{rc} 02$ & 50128154 & 50130890 & NBS-LRR & & P-loop[198-206] Kin-2[280-287] RNBS-B[307-313] GLPL[371-377] & 630]LRR_8:0.0021 \\
\hline RC2G0432000 & $\mathrm{rc} 02$ & 50213957 & 50215216 & NBS & & P-loop[18-26] Kin-2[100-107] RNBS-B[127-133] GLPL[191-197] & $\begin{array}{l}\text { [5-245]NB-ARC:3.5e-61 } \\
\text { [105-395]NB-ARC:6.5e-60 [497- } \\
\text { 543]LRR_8:3.1e-06 [544-562]LRR_8:1.2e- }\end{array}$ \\
\hline RC2G0439500 & $\mathrm{rc02}$ & 51259407 & 51261938 & NBS-LRR & & P-loop[134-142] Kin-2[214-221] RNBS-B[243-249] GLPL[306-312] & $\begin{array}{l}05 \\
\text { [184-448]NB-ARC:2.3e-59 [553- } \\
\text { 562]LRR_8:0.00013 [563- } \\
\text { 617]LRR_8:1.6e-05 [766- }\end{array}$ \\
\hline RC2G0441000 & $\mathrm{rc02}$ & 51485056 & 51489326 & NBS-LRR & & P-loop[194-200] Kin-2[271-278] RNBS-B[300-306] GLPL[359-365] & $\begin{array}{l}\text { 796]LRR_4:0.0049 } \\
\text { [160-437]NB-ARC:3.9e-87 [518- }\end{array}$ \\
\hline RC2G0446800 & $\mathrm{rc02}$ & 52027088 & 52029827 & CC-NBS-LRR & & P-loop[182-190] Kin-2[260-267] RNBS-B[287-293] GLPL[347-353] & $\begin{array}{l}\text { 535]LRR_9:0.0018 [536-594]LRR_8:1.1e- } \\
\text { 11 [595-624]LRR_4:0.00018 } \\
\text { [181-203]AAA_16:9.6e-05 [204-467]NB- } \\
\text { ARC:1.3e-60 [564-588]LRR_8:2.8e-05 } \\
\text { [589-644]LRR_8:2.6e-06 [644- } \\
\text { 668]LRR_8:0.00052 [778- }\end{array}$ \\
\hline RC2G0474400 & $\mathrm{rc02}$ & 55058308 & 55061000 & CC-NBS-LRR & & P-loop[214-222] Kin-2[287-294] RNBS-B[316-322] GLPL[378-384] & $\begin{array}{l}\text { 813]LRR_8:0.00043 } \\
\text { [182-201]AAA_16:0.00062 [202-468]NB- } \\
\text { ARC:2.3e-59 [593-648]LRR_8:5.6e-06 }\end{array}$ \\
\hline RC2G0474900 & $\mathrm{rc02}$ & 55134538 & 55137227 & CC-NBS-LRR & & P-loop[215-223] Kin-2[288-295] RNBS-B[317-323] GLPL[379-385] & [648-670]LRR_8:0.0023 \\
\hline
\end{tabular}




\begin{tabular}{|c|c|c|c|c|c|}
\hline RC2G0506600 & $\mathrm{rc} 02$ & 58862239 & 58866940 & NBS & DAGK_c \\
\hline RC2G0506700 & $\mathrm{rc} 02$ & 58868511 & 58869206 & NBS & \\
\hline RC2G0534600 & $\mathrm{rc} 02$ & 61705745 & 61710532 & NBS & \\
\hline RC2G0587100 & $\mathrm{rc} 02$ & 66525159 & 66528095 & CC-NBS-LRR & \\
\hline RC2G0588700 & $\mathrm{rc} 02$ & 66625748 & 66630124 & CC-NBS-LRR & \\
\hline RC2G0589000 & $\mathrm{rc} 02$ & 66647790 & 66651098 & CC-NBS-LRR & \\
\hline RC2G0620500 & $\mathrm{rc} 02$ & 69511698 & 69514439 & NBS-LRR & \\
\hline RC2G0620800 & $\mathrm{rc} 02$ & 69557522 & 69560264 & NBS-LRR & \\
\hline RC3G0032000 & $\mathrm{rc} 03$ & 6974322 & 6978370 & NBS-LRR & \\
\hline RC3G0032300 & $\mathrm{rc} 03$ & 7001366 & 7009066 & NBS & \\
\hline RC3G0034200 & $\mathrm{rc} 03$ & 7189856 & 7196786 & NBS-LRR & \\
\hline RC3G0042600 & $\mathrm{rc} 03$ & 8370125 & 8377814 & TIR-NBS-LRR & \\
\hline RC3G0044100 & $\mathrm{rc} 03$ & 8494667 & 8497519 & CC-NBS-LRR & \\
\hline RC3G0047600 & $\mathrm{rc} 03$ & 9080520 & 9088487 & TIR-NBS-LRR & \\
\hline RC3G0062300 & $\mathrm{rc} 03$ & 11919385 & 11921083 & NBS-LRR & \\
\hline RC3G0070000 & $\mathrm{rc} 03$ & 12642166 & 12647039 & NBS & RPW8 \\
\hline RC3G0070100 & $\mathrm{rc} 03$ & 12670256 & 12677491 & NBS-LRR & RPW8 \\
\hline RC3G0075200 & $\mathrm{rc} 03$ & 13340577 & 13343332 & NBS & \\
\hline RC3G0098600 & $\mathrm{rc} 03$ & 17063773 & 17066571 & TIR-NBS & \\
\hline RC3G0111000 & $\mathrm{rc} 03$ & 18591350 & 18592827 & NBS-LRR & \\
\hline RC3G0123500 & $\mathrm{rc} 03$ & 20179686 & 20181286 & TIR-NBS & \\
\hline RC3G0124000 & $\mathrm{rc} 03$ & 20217168 & 20219331 & TIR-NBS & \\
\hline RC3G0124200 & $\mathrm{rc} 03$ & 20245230 & 20250243 & TIR-NBS-LRR & \\
\hline RC3G0136400 & $\mathrm{rc} 03$ & 21740920 & 21744430 & NBS & RPW8 \\
\hline RC3G0136500 & $\mathrm{rc} 03$ & 21762759 & 21766253 & NBS & RPW8 \\
\hline
\end{tabular}

[11-247]NB-ARC:8.8e-20 [513-

617]DAGK_acc: $1.2 \mathrm{e}-10$

[1-221]NB-ARC:3e-19

P-loop[1-8] Kin-2[90-97] RNBS-B[131-137] GLPL[202-208]

P-loop[1-8] Kin-2[89-96] RNBS-B[118-124] GLPL[180-186]

[1-268]NB-ARC:6.1e-57

[189-476]NB-ARC:7.9e-64 [574-

608]LRR_8:0.0082 [610-

P-loop[217-225] Kin-2[294-301] RNBS-B[325-331] GLPL[387-393] 651]LRR_8:0.0058

[188-474]NB-ARC:1.3e-60 [572-

629]LRR_8:2.1e-07 [629-

P-loop[216-224] Kin-2[293-300] RNBS-B[324-330] GLPL[385-391] 653]LRR_8:0.0015

[191-474]NB-ARC:7.7e-63 [574-

631]LRR_8:1.2e-08 [631-652]LRR_8:2e-

P-loop[215-223] Kin-2[292-299] RNBS-B[323-329] GLPL[385-391] 07

P-loop[185-193] Kin-2[258-265] RNBS-B[284-290] GLPL[346-352] [163-430]NB-ARC:2.5e-51

P-loop[185-193] Kin-2[258-265] RNBS-B[284-290] GLPL[346-352] [163-430]NB-ARC:2.4e-51

P-loop[205-213] Kin-2[280-287] RNBS-B[311-317] GLPL[372-378]

[177-459]NB-ARC:6.1e-61 [539-

P-loop[205-213] Kin-2[280-287] RNBS-B[311-317] GLPL[372-378] [177-378]NB-ARC:1.3e-42 [177-459]NB-ARC:8.2e-61 [539574]LRR_4:0.0035 [843-

P-loop[205-213] Kin-2[280-287] RNBS-B[311-317] GLPL[372-378] 903]LRR_8:0.00019

[24-71]TIR:4.5e-10 [72-141]TIR:0.0014

[157-411]NB-ARC:4.7e-33 [706-

P-loop[181-189] Kin-2[260-267] RNBS-B[287-293] GLPL[346-352] 738]LRR_8:0.00025

[181-192]AAA_16:0.00023 [193-467]NB-

P-loop[212-220] Kin-2[289-296] RNBS-B[318-324] GLPL[379-385] ARC:2.5e-63 [620-660]LRR_4:0.001

[23-193]TIR:2.9e-46 [327-

344]AAA_16:0.00033 [345-613]NB-

P-loop[358-366] Kin-2[433-440] RNBS-B[462-468] GLPL[523-529] ARC:6.1e-66 [731-786]LRR_8:3.8e-05

[44-320]NB-ARC:1.3e-49 [416-

P-loop[65-73] Kin-2[140-147] RNBS-B[169-175] GLPL[229-235] 474]LRR_8:2.2e-11

[16-136]RPW8:9e-14 [159-445]NB-

P-loop[180-188] Kin-2[279-286] RNBS-B[305-311] GLPL[360-366] ARC:1.1e-33

[5-111]RPW8:3.9e-13 [151-406]NB-

P-loop[166-174] Kin-2[243-250] RNBS-B[271-277] GLPL[325-331] ARC:7.5e-30

P-loop[177-185] Kin-2[251-258] RNBS-B[282-283] GLPL[344-350] [155-435]NB-ARC:2.7e-60

[9-174]TIR:7.4e-48 [182-443]NB-

P-loop[206-214] Kin-2[284-291] RNBS-B[311-317] GLPL[369-375] ARC:4.8e-25 [600-615]LRR_3:0.0087

[14-281]NB-ARC:1.9e-54 [385-

P-loop[35-43] Kin-2[110-117] RNBS-B[139-145] GLPL[198-204]

443]LRR_8:2.4e-08

[7-100]TIR:2.2e-12 [113-346]NB-

P-loop[125-133] Kin-2[196-203] RNBS-B[223-229] GLPL[282-288] ARC:3.2e-36

[22-205]TIR:5.5e-47 [208-

217]AAA_16:0.0078 [218-468]NB-

P-loop[230-238] Kin-2[302-309] RNBS-B[329-335] GLPL[388-394] ARC:2.7e-34 [609-626]LRR_3:0.0038

[22-205]TIR:7.5e-46 [219-456]NBARC:1.2e-33 [610-627]LRR_3:0.0008 [842-901]LRR_8:1.9e-05 [901-

P-loop[230-238] Kin-2[302-309] RNBS-B[329-335] GLPL[388-394] 911]LRR_4:0.001

[1-101]RPW8:3.6e-18 [142-407]NB-

P-loop[161-169] Kin-2[240-247] RNBS-B[266-272] GLPL[321-327] ARC:1.3e-31

[3-80]RPW8:1.3e-07 [122-385]NB-

P-loop[139-147] Kin-2[218-225] RNBS-B[244-250] GLPL[299-305] ARC:3.1e-30 


\begin{tabular}{|c|c|c|c|c|c|c|c|}
\hline RC3G0136600 & $\mathrm{rc} 03$ & 21808068 & 21810533 & NBS & & P-loop[82-90] Kin-2[157-164] RNBS-B[187-193] GLPL[240-244] & [62-325]NB-ARC:6.6e-20 \\
\hline RC3G0145900 & $\mathrm{rc} 03$ & 22703075 & 22704352 & CC-NBS & & P-loop[178-186] Kin-2[267-274] RNBS-B[308-314] GLPL[371-377] & $\begin{array}{l}\text { [169-386]NB-ARC:3e-29 } \\
{[43-319] N B-A R C: 3.1 \mathrm{e}-48 \text { [413- }}\end{array}$ \\
\hline RC3G0199200 & $\mathrm{rc} 03$ & 28299616 & 28301075 & NBS-LRR & & P-loop[65-73] Kin-2[140-147] RNBS-B[169-175] GLPL[228-234] & $\begin{array}{l}\text { 447]LRR_8:5.3e-05 } \\
{[14-289] \text { NB-ARC:8e-54 [383- }}\end{array}$ \\
\hline RC3G0200100 & $\mathrm{rc} 03$ & 28424037 & 28425715 & NBS-LRR & & P-loop[35-43] Kin-2[110-117] RNBS-B[139-145] GLPL[198-204] & $\begin{array}{l}\text { 441]LRR_8:1.1e-09 } \\
\text { [3-84]TIR:1.1e-07 [105-380]NB-ARC:1.6e- }\end{array}$ \\
\hline RC3G0200400 & $\mathrm{rc} 03$ & 28514921 & 28516507 & TIR-NBS & & P-loop[127-135] Kin-2[202-209] RNBS-B[231-237] GLPL[290-296] & $\begin{array}{l}48 \\
{[2-123] \mathrm{TIR}: 2.6 \mathrm{e}-27[138-413] \mathrm{NB}-}\end{array}$ \\
\hline RC3G0200800 & $\mathrm{rc} 03$ & 28549670 & 28551783 & TIR-NBS & & P-loop[160-168] Kin-2[235-242] RNBS-B[264-270] GLPL[323-329] & $\begin{array}{l}\text { ARC:1.4e-51 } \\
\text { [14-292]NB-ARC:1.4e-48 [385- }\end{array}$ \\
\hline RC3G0201400 & $\mathrm{rc} 03$ & 28584130 & 28585769 & NBS-LRR & & P-loop[36-44] Kin-2[111-118] RNBS-B[140-146] GLPL[200-206] & 443]LRR_8:1.4e-10 \\
\hline RC3G0207900 & $\mathrm{rc} 03$ & 29140368 & 29142896 & CC-NBS-LRR & RPW8 & P-loop[184-192] Kin-2[258-265] RNBS-B[287-293] GLPL[344-350] & $\begin{array}{l}\text { [15-89]RPW8:0.0049 [154-432]NB- } \\
\text { ARC:1.6e-53 [551-605]LRR_8:4.2e-08 }\end{array}$ \\
\hline RC3G0216100 & $\mathrm{rc} 03$ & 30235476 & 30237794 & NBS-LRR & & P-loop[74-82] Kin-2[145-152] RNBS-B[173-179] GLPL[226-232] & [56-310]NB-ARC:5.7e-32 \\
\hline RC3G0242300 & $\mathrm{rc} 03$ & 33188950 & 33190098 & NBS & & P-loop[205-213] Kin-2[280-287] RNBS-B[311-317] GLPL[372-378] & $\begin{array}{l}{[177-379] N B-A R C: 4.9 e-39} \\
{[176-459] N B-A R C \cdot 97 e-59[1294-}\end{array}$ \\
\hline RC3G0243600 & $\mathrm{rc} 03$ & 33281601 & 33296322 & NBS-LRR & Cyclin_N & P-loop[204-212] Kin-2[279-286] RNBS-B[310-316] GLPL[371-377] & $\begin{array}{l}\text { 1344]Cyclin_N:6.4e-07 } \\
\text { [193-460]NB-ARC:1.7e-55 [532- } \\
\text { 586]LRR_8:0.00013 [878- } \\
\text { 936]LRR_8:0.0033 [975- } \\
\text { 1012]LRR_4:0.00067 }\end{array}$ \\
\hline RC3G0244300 & $\mathrm{rc} 03$ & 33345473 & 33348282 & NBS & & P-loop[222-230] Kin-2[296-303] RNBS-B[327-333] GLPL[388-394] & $\begin{array}{l}\text { [194-465]NB-ARC:4.1e-53 } \\
{[171-181] \text { AAA_16:9.4e-05 [182-457]NB- }}\end{array}$ \\
\hline RC3G0249600 & $\mathrm{rc} 03$ & 33811859 & 33815053 & NBS & & P-loop[203-211] Kin-2[278-285] RNBS-B[309-315] GLPL[370-376] & $\begin{array}{l}\text { ARC: } 4.8 \mathrm{e}-62 \\
{[2-280] N B-A R C: 6.7 e-60[353-}\end{array}$ \\
\hline RC3G0252800 & $\mathrm{rc} 03$ & 34112771 & 34115901 & NBS-LRR & & P-loop[23-31] Kin-2[98-105] RNBS-B[129-135] GLPL[190-196] & $\begin{array}{l}\text { 389]LRR_8:0.005 } \\
\text { [23-182]TIR:4.3e-50 [202- } \\
\text { 215]AAA_16:0.0022 [216-455]NB- } \\
\text { ARC:3.6e-31 [609-626]LRR_3:6.9e-05 } \\
\text { [726-782]LRR_8:0.0057 [863- }\end{array}$ \\
\hline RC3G0272000 & $\mathrm{rc} 03$ & 35710822 & 35716742 & TIR-NBS-LRR & & P-loop[227-235] Kin-2[305-312] RNBS-B[332-338] GLPL[391-397] & 918]LRR_8:0.00023 \\
\hline RC3G0277900 & $\mathrm{rc} 03$ & 36251527 & 36255728 & CC-NBS-LRR & & P-loop[205-213] Kin-2[280-287] RNBS-B[311-317] GLPL[372-378] & $\begin{array}{l}\text { [181-459]NB-ARC:9e-57 } \\
{[177-459] \text { NB-ARC:7.4e-61 [876- }}\end{array}$ \\
\hline RC3G0280800 & $\mathrm{rc} 03$ & 36402106 & 36405245 & NBS-LRR & & P-loop[204-212] Kin-2[279-286] RNBS-B[310-316] GLPL[371-377] & \\
\hline RC3G0357100 & $\mathrm{rc} 03$ & 42585842 & 42591797 & NBS-LRR & & P-loop[79-87] Kin-2[164-171] RNBS-B[191-197] GLPL[250-256] & $\begin{array}{l}\text { 719]LRR_8:0.00049 } \\
\text { [185-468]NB-ARC:3e-57 [627- }\end{array}$ \\
\hline RC3G0380200 & $\mathrm{rc} 03$ & 44011689 & 44014786 & NBS-LRR & & P-loop[212-220] Kin-2[291-298] RNBS-B[322-328] GLPL[384-390] & $\begin{array}{l}\text { 660]LRR_8:0.00083 } \\
\text { [183-468]NB-ARC:1.6e-54 [574- } \\
\text { 600]LRR_8:0.0063 [605- } \\
\text { 635]LRR_4:0.00079 [651- } \\
\text { 682]LRR_4:0.0008 [1035- } \\
\text { 1129]LRR_5:0.0031 [1129- }\end{array}$ \\
\hline RC3G0380700 & $\mathrm{rc} 03$ & 44048794 & 44052609 & NBS-LRR & & P-loop[208-216] Kin-2[289-296] RNBS-B[320-326] GLPL[381-387] & $\begin{array}{l}\text { 1158]LRR_4:0.0038 } \\
\text { [187-473]NB-ARC:5.7e-57 [603- } \\
\text { 647]LRR_8:0.0011 [648- } \\
\text { 665]LRR_4:0.0013 [1016- }\end{array}$ \\
\hline RC3G0380800 & $\mathrm{rc} 03$ & 44079169 & 44082987 & NBS-LRR & & P-loop[214-222] Kin-2[293-300] RNBS-B[324-330] GLPL[386-392] & 1134]LRR_5:5.2e-06 \\
\hline RC3G0380900 & $\mathrm{rc} 03$ & 44084061 & 44085554 & NBS & & P-loop[207-215] Kin-2[286-293] RNBS-B[317-323] GLPL[379-385] & {$[180-453] N B-A R C: 4.5 e-49$} \\
\hline RC4G0003800 & $\mathrm{rc} 04$ & 307316 & 310088 & NBS-LRR & & P-loop[6-14] Kin-2[82-89] RNBS-B[109-115] GLPL[168-174] & $\begin{array}{l}\text { [1-242]NB-ARC:9.9e-33 [440- } \\
\text { 496]LRR_8:3e-05 [536-558]LRR_8:0.0008 } \\
\text { [559-589]LRR_4:0.00014 [637- } \\
\text { 696]LRR_8:6.8e-05 }\end{array}$ \\
\hline
\end{tabular}


851]LRR_8:0.0032 [913-

969]LRR_8:0.0049 [1008-

1062]LRR_8:0.0074 [1115-

$\begin{array}{lrrrl}\text { RC4G0006200 } & \mathrm{rc04} & 498278 & 503605 & \text { NBS-LRR } \\ \text { RC4G0009500 } & \mathrm{rc04} & 713187 & 716014 & \text { NBS-LRR } \\ \text { RC4G0018200 } & \mathrm{rc04} & 1569721 & 1571754 & \text { NBS-LRR } \\ & & & & \\ \text { RC4G0026100 } & \mathrm{rc04} & 2528716 & 2531583 & \text { CC-NBS-LRR } \\ & & & & \\ \text { RC4G0026700 } & \mathrm{rc04} & 2595101 & 2603424 & \text { TIR-NBS } \\ & & & & \\ \text { RC4G0027200 } & \mathrm{rc04} & 2627151 & 2630401 & \text { TIR-NBS-LRR }\end{array}$

P-loop[81-89] Kin-2[154-161] RNBS-B[181-187] GLPL[240-246]

P-loop[2-10] Kin-2[75-82] RNBS-B[102-108] GLPL[161-167]

P-loop[6-14] Kin-2[81-88] RNBS-B[108-114] GLPL[167-173]

P-loop[212-220] Kin-2[286-293] RNBS-B[315-321] GLPL[376-382]

P-loop[232-240] Kin-2[305-312] RNBS-B[332-338] GLPL[391-397]

P-loop[232-240] Kin-2[308-315] RNBS-B[335-341] GLPL[394-400]

\section{P-loop[6-14] Kin-2[82-89] RNBS-B[109-115] GLPL[168-174]}

P-loop[6-14] Kin-2[82-89] RNBS-B[109-115] GLPL[168-174]

P-loop[178-186] Kin-2[252-259] RNBS-B[280-286] GLPL[344-350] P-loop[178-186] Kin-2[232-239] RNBS-B[260-266] GLPL[324-330] P-loop[178-186] Kin-2[252-259] RNBS-B[280-286] GLPL[344-350] P-loop[193-201] Kin-2[274-281] RNBS-B[302-308] GLPL[368-374] P-loop[193-201] RNBS-B[284-290] GLPL[343-349]

P-loop[193-201] Kin-2[274-281] RNBS-B[302-308] GLPL[368-374] $10595425 \quad 10611786$ CC-NBS-LRR 10659086
10661392 CC-NBS-LRR
$6519333 \quad 6524016 \quad$ NBS-LRR

$8903303 \quad 8907438 \quad$ CC-NBS-LRR

$10344840 \quad 10346607 \quad$ CC-NBS
]LRR_4:0.0019

[2-234]NB-ARC:2.7e-36 [412-

447]LRR_4:0.00031

[1-232]NB-ARC:5.5e-34 [420455]LRR_4:0.004

[188-461]NB-ARC:3.5e-62 [591-

617]LRR_8:0.00056 [618-

658]LRR_4:0.00037 [794

826]LRR_4:0.00096

[29-190]TIR:5.3e-45 [205-

215]AAA_16:0.00018 [216-456]NB-

ARC:1.1e-36

[30-188]TIR:3.5e-45 [214-467]NB-

ARC:4.5e-38 [649-691]LRR_4:9.7e-05

[694-727]LRR_4:0.0017 [789-

845]LRR 8:5.3e-05

[1-233]NB-ARC:4.7e-34 [419-

451]LRR_4:0.0058 [455-498]LRR_4:3.4e-

07 [501-543]LRR_4:1.1e-05 [553-

590]LRR_4:0.00034 [711-

767]LRR_8:0.00048 [783-

838]LRR_8:0.00016 [861-921]LRR_8:2e06

[1-240]NB-ARC:4.6e-34 [411-

452]LRR_4:0.00024 [456-

498]LRR_4:3.4e-05 [502-545]LRR_4:6e08 [548-583]LRR_4:0.00089 [643-

698]LRR_8:0.0039 [714-

767]LRR_8:0.0071

[155-436]NB-ARC:1.9e-73

[155-416]NB-ARC:5.7e-58

[155-436]NB-ARC:1.7e-73

[169-465]NB-ARC:4.7e-74 [809-

845]LRR_8:0.0017

[169-439]NB-ARC:1e-60 [766-

799]LRR_4:0.0052

[169-464]NB-ARC:7.3e-74 [810-

$\mathrm{RC} 4 \mathrm{G} 0085600 \quad \mathrm{rc} 04$

rc04

RC4G0088900 rc04
P-loop[188-196] Kin-2[265-272] RNBS-B[289-295] GLPL[348-354] P-loop[178-186] Kin-2[255-262] RNBS-B[279-285] GLPL[338-344] P-loop[186-194] Kin-2[263-270] RNBS-B[287-293] GLPL[346-352]

]LRR_4:0.00048

[167-415]NB-ARC:1.7e-48 [629-

664]LRR 8:0.0015

[157-411]NB-ARC:1.9e-48

[167-413]NB-ARC:2.2e-44 [535-

588]LRR_8:0.00012

[6-110]DUF677:0.00047 [177-439]NBARC:3.6e-46 [557-601]LRR_8:0.0036

P-loop[195-203] Kin-2[272-279] RNBS-B[296-302] GLPL[355-361] $\quad$ [635-670]LRR_4:0.0043 


\begin{tabular}{|c|c|c|c|c|c|c|c|}
\hline RC4G0089900 & rc04 & 10762137 & 10766873 & CC-NBS & & P-loop[184-192] Kin-2[262-269] RNBS-B[286-292] GLPL[345-351] & $\begin{array}{l}\text { [166-417]NB-ARC:2.6e-46 } \\
{[112-378] N B-A R C: 4.3 e-44 \text { [491- }}\end{array}$ \\
\hline RC4G0092500 & $\mathrm{rc} 04$ & 11062966 & 11078607 & NBS-LRR & & P-loop[130-138] Kin-2[207-214] RNBS-B[231-237] GLPL[290-296] & $\begin{array}{l}\text { 535]LRR_8:0.00064 } \\
\text { [163-495]NB-ARC:1.1e-63 [623- }\end{array}$ \\
\hline RC4G0101000 & rc04 & 12580676 & 12583891 & NBS-LRR & & P-loop[186-194] Kin-2[315-322] RNBS-B[342-348] GLPL[402-408] & $\begin{array}{l}\text { 667]LRR_8:0.0083 } \\
\text { [20-188]TIR:6.3e-49 [201-458]NB- }\end{array}$ \\
\hline RC4G0131400 & $\mathrm{rc} 04$ & 18217613 & 18221438 & TIR-NBS-LRR & & P-loop[224-232] Kin-2[303-310] RNBS-B[330-336] GLPL[389-395] & $\begin{array}{l}\text { ARC:1.4e-33 [820-875]LRR_8:4e-07 [876- } \\
\text { 886]LRR_4:0.0011 } \\
\text { [159-435]NB-ARC:3.2e-84 [532- } \\
\text { 569]LRR_8:8.9e-08 [579- }\end{array}$ \\
\hline RC4G0150300 & $\mathrm{rc} 04$ & 21839855 & 21844046 & CC-NBS-LRR & & P-loop[180-188] Kin-2[258-265] RNBS-B[285-291] GLPL[345-351] & $\begin{array}{l}\text { 614]LRR_8:0.00017 } \\
\text { [162-454]NB-ARC:3e-70 [618- } \\
\text { 670]LRR_8:0.00066 [783- }\end{array}$ \\
\hline RC4G0150700 & rc04 & 21950838 & 21954395 & NBS-LRR & & P-loop[186-194] Kin-2[267-274] RNBS-B[294-300] GLPL[359-365] & 817]LRR_4:0.0087 \\
\hline RC4G0156700 & $\mathrm{rc} 04$ & 22960778 & 22965591 & CC-NBS-LRR & & P-loop[346-354] Kin-2[423-430] RNBS-B[447-453] GLPL[506-512] & $\begin{array}{l}\text { [326-588]NB-ARC:3.9e-48 [761- } \\
\text { 816]LRR_8:0.0091 }\end{array}$ \\
\hline RC4G0161100 & $\mathrm{rc} 04$ & 23754452 & 23761269 & NBS-LRR & & P-loop[196-204] Kin-2[271-278] RNBS-B[301-307] GLPL[358-364] & $\begin{array}{l}\text { [163-184]AAA_16:5.3e-05 [185-448]NB- } \\
\text { ARC:6.7e-63 [555-602]LRR_8:0.00015 } \\
\text { [172-459]NB-ARC:1e-83 [576- }\end{array}$ \\
\hline RC4G0177100 & $\mathrm{rc} 04$ & 26269454 & 26272936 & CC-NBS-LRR & & P-loop[194-202] Kin-2[276-283] RNBS-B[303-309] GLPL[367-373] & $\begin{array}{l}\text { 631]LRR_8:7.2e-06 } \\
\text { [36-142]RPW8:3.5e-14 [241-508]NB- }\end{array}$ \\
\hline RC4G0185400 & $\mathrm{rc} 04$ & 27299484 & 27309739 & CC-NBS-LRR & RPW8 & P-loop[260-268] Kin-2[337-344] RNBS-B[367-373] GLPL[421-427] & $\begin{array}{l}\text { ARC:2.6e-33 [777-799]LRR_8:0.0087 } \\
\text { [800-838]LRR_4:0.0034 } \\
\text { [17-264]NB-ARC:8.3e-34 [534- } \\
\text { 554]LRR_8:0.0054 [555- } \\
\text { 577]LRR_4:0.00055 [578- } \\
\text { 634]LRR_8:3.7e-07 [640- } \\
\text { 677]LRR_8:0.0021 [692- }\end{array}$ \\
\hline RC4G0186600 & rc04 & 27506043 & 27509702 & NBS-LRR & & P-loop[35-43] Kin-2[114-121] RNBS-B[141-147] GLPL[200-206] & $\begin{array}{l}\text { 747]LRR_8:0.0019 } \\
\text { [21-187]TIR:1.7e-47 [206-453]NB- } \\
\text { ARC:1.8e-32 [608-663]LRR_8:0.0026 } \\
\text { [748-770]LRR_4:0.0077 [771- }\end{array}$ \\
\hline RC4G0188900 & $\mathrm{rc} 04$ & 27670968 & 27676905 & TIR-NBS-LRR & & P-loop[227-235] Kin-2[306-313] RNBS-B[333-339] GLPL[392-398] & $\begin{array}{l}\text { 815]LRR_8:0.00073 [816- } \\
\text { 855]LRR_4:1.2e-05 [856-872]LRR_8:1.3e- } \\
05 \text { [987-1021]LRR_8:0.0082 }\end{array}$ \\
\hline RC4G0191600 & $\mathrm{rc} 04$ & 28026959 & 28031459 & TIR-NBS-LRR & & P-loop[219-227] Kin-2[298-305] RNBS-B[325-331] GLPL[384-390] & $\begin{array}{l}\text { [14-183]TIR:5.5e-47 [196-450]NB- } \\
\text { ARC:1e-30 [833-873]LRR_4:0.00014 } \\
\text { [173-182]AAA_16:0.0014 [183-460]NB- } \\
\text { ARC:4.2e-60 [876-931]LRR_8:0.001 }\end{array}$ \\
\hline RC4G0192500 & $\mathrm{rc} 04$ & 28192080 & 28197651 & NBS-LRR & & P-loop[204-212] Kin-2[279-286] RNBS-B[310-316] GLPL[371-377] & $\begin{array}{l}\text { [1156-1195]LRR_4:0.0015 } \\
\text { [1-234]NB-ARC:2.3e-30 [524- } \\
\text { 563]LRR_4:0.0015 [570- } \\
\text { 593]LRR_4:0.0045 [594- } \\
\text { 640]LRR_8:0.0025 [641- }\end{array}$ \\
\hline RC4G0206200 & $\mathrm{rc} 04$ & 30253977 & 30257175 & NBS-LRR & & P-loop[6-14] Kin-2[84-91] RNBS-B[111-117] GLPL[170-176] & $\begin{array}{l}\text { 696]LRR_8:0.00059 } \\
\text { [1-215]NB-ARC:1.4e-19 [488- } \\
\text { 543]LRR_8:0.00054 [580-- } \\
\text { 636]LRR_8:0.00091 [672- }\end{array}$ \\
\hline RC4G0206500 & $\mathrm{rc} 04$ & 30288798 & 30294008 & NBS-LRR & & P-loop[6-14] Kin-2[84-91] RNBS-B[111-117] GLPL[154-160] & $\begin{array}{l}\text { 727]LRR_8:0.0013 } \\
\text { [169-461]NB-ARC:1.5e-59 [568- }\end{array}$ \\
\hline RC4G0208800 & rc04 & 30532282 & 30535890 & CC-NBS-LRR & & P-loop[196-204] Kin-2[272-279] RNBS-B[303-309] GLPL[370-376] & $\begin{array}{l}\text { 622]LRR_8:1.8e-05 } \\
\text { [176-462]NB-ARC:7.5e-62 [521- } \\
\text { 571]LRR_8:1.8e-06 [895- } \\
\text { 919]LRR_8:0.00054 [920- } \\
\text { 978]LRR_8:0.00034 [991- } \\
\text { 1093]LRR_5:4.1e-07 }\end{array}$ \\
\hline
\end{tabular}




\begin{tabular}{|c|c|c|c|c|}
\hline RC4G0211800 & rc04 & 30991554 & 30994632 & NBS \\
\hline RC4G0232800 & $\mathrm{rc04}$ & 34153012 & 34155878 & NBS-LRR \\
\hline RC4G0233100 & $\mathrm{rc} 04$ & 34202653 & 34205479 & NBS-LRR \\
\hline RC4G0233500 & $\mathrm{rc} 04$ & 34258520 & 34261161 & NBS-LRR \\
\hline RC4G0233700 & $\mathrm{rc} 04$ & 34301613 & 34305036 & NBS-LRR \\
\hline RC4G0238500 & $\mathrm{rc} 04$ & 34800183 & 34810214 & NBS-LRR \\
\hline RC4G0239200 & $\mathrm{rc} 04$ & 34918326 & 34920024 & NBS \\
\hline RC4G0239400 & $\mathrm{rc} 04$ & 34935796 & 34938486 & NBS-LRR \\
\hline RC4G0239800 & $\mathrm{rc} 04$ & 35002789 & 35005494 & NBS-LRR \\
\hline RC4G0240000 & $\mathrm{rc} 04$ & 35027048 & 35030761 & NBS-LRR \\
\hline RC4G0240400 & $\mathrm{rc} 04$ & 35053190 & 35054891 & NBS \\
\hline RC4G0248700 & $\mathrm{rc} 04$ & 36192025 & 36195770 & NBS-LRR \\
\hline RC4G0249100 & $\mathrm{rc} 04$ & 36239268 & 36244166 & NBS-LRR \\
\hline RC4G0249700 & $\mathrm{rc04}$ & 36295031 & 36299798 & NBS-LRR \\
\hline
\end{tabular}

$\mathrm{RC} 4 \mathrm{G} 0249900 \quad \mathrm{rc} 04$

RC4G0250200 rc04

RC4G0279600 rc04

$\mathrm{RC} 4 \mathrm{G} 0280400 \quad \mathrm{rc} 04$

RC4G0281100 rc04
$36308849 \quad 36319578 \quad$ NBS-LRR

$36351503 \quad 36355784 \quad$ NBS-LRR

$39822263 \quad 39826573 \quad$ NBS-LRR

$39888481 \quad 39889072 \quad$ NBS

$39962829 \quad 39966458 \quad$ NBS
P-loop[201-209] Kin-2[276-283] RNBS-B[307-313] GLPL[366-372] [175-419]NB-ARC:8.1e-50 [183-474]NB-ARC:2.1e-61 [577608]LRR_8:0.0026 [609-

P-loop[210-218] Kin-2[289-296] RNBS-B[320-326] GLPL[381-387] 647]LRR_8:0.00098

[113-404]NB-ARC:4.5e-62 [773-

P-loop[140-148] Kin-2[219-226] RNBS-B[250-256] GLPL[311-317] 813]LRR_4:0.0048

[113-404]NB-ARC:2.1e-58 [503-

533]LRR_8:0.00042 [534-

573]LRR 8:8.8e-05 [730-

P-loop[140-148] Kin-2[219-226] RNBS-B[250-256] GLPL[311-317] 806]LRR_5:0.0015

[113-402]NB-ARC:3.4e-60 [520-

P-loop[138-146] Kin-2[217-224] RNBS-B[248-254] GLPL[309-315]

575]LRR_8:0.00017

[1-265]NB-ARC:4.1e-58 [387-

436]LRR_4:0.00092 [437-

452]LRR_8:0.008 [713-735]LRR_8:0.0008

[736-775]LRR_4:0.00023 [877-

P-loop[5-13] Kin-2[83-90] RNBS-B[114-120] GLPL[175-181] 918]LRR 4:0.0094

P-loop[212-220] Kin-2[290-297] RNBS-B[321-327] GLPL[382-388] [187-474]NB-ARC:2.5e-55 [62-353]NB-ARC:8.5e-60 [462499]LRR_4:0.00048 [500

P-loop[89-97] Kin-2[168-175] RNBS-B[199-205] GLPL[260-266]

513]LRR_8:0.00064

[1-269]NB-ARC:1.6e-58 [373-

421]LRR_8:0.0023 [422-

439]LRR_4:0.0024 [636-

P-loop[5-13] Kin-2[84-91] RNBS-B[115-121] GLPL[176-182]

LRR_4:0.00071

[1-268]NB-ARC:1e-56 [381-

422]LRR_4:0.0023 [423

P-loop[5-13] Kin-2[83-90] RNBS-B[114-120] GLPL[175-181]

436]LRR_8:0.0039

[59-79]AAA_16:0.0025 [80-349]NB-

ARC:6.6e-58

P-loop[89-97] Kin-2[167-174] RNBS-B[198-204] GLPL[259-265

[183-473]NB-ARC:2.7e-61 [621-

P-loop[209-217] Kin-2[287-294] RNBS-B[318-324] GLPL[380-386] 668]LRR_8:0.0074

[183-472]NB-ARC:4.8e-64 [582-

591]LRR_8:3.8e-05 [592-630]LRR_4:3.3e-

05 [631-644]LRR_8:0.00032 [926-

P-loop[210-218] Kin-2[288-295] RNBS-B[319-325] GLPL[380-386] 965]LRR_4:0.00016

[182-471]NB-ARC:9.6e-64 [555-

593]LRR 4:0.0017 [887

P-loop[209-217] Kin-2[287-294] RNBS-B[318-324] GLPL[379-385] 926]LRR_4:0.0021

[182-472]NB-ARC:3.1e-64 [713-

779]gag_pre-integrs:1.5e-14 [792-

904]rve:1.3e-22 [1218-1461]RVT_2:2.1e-

gag_pre-

integrs,rve,

RVT_2

P-loop[209-217] Kin-2[287-294] RNBS-B[318-324] GLPL[379-385]

2044]LRR 5:0.0016 [2045-

2084]LRR 4:0.0015

[183-473]NB-ARC:3.1e-64 [582-

591]LRR_8:0.00096 [592-

629]LRR_4:0.00034 [1043-

1101]LRR_8:0.0022 [1126-

P-loop[210-218] Kin-2[288-295] RNBS-B[319-325] GLPL[380-386]

LRR 5:0.0031

[175-186]AAA_16:0.0074 [187-459]NB-

P-loop[205-213] Kin-2[280-287] RNBS-B[311-317] GLPL[372-378] ARC:5.2e-57

P-loop[1-8] Kin-2[75-82] RNBS-B[106-112] GLPL[167-173] [1-176]NB-ARC:3.5e-34

P-loop[205-213] Kin-2[280-287] RNBS-B[311-317] GLPL[372-378] [177-460]NB-ARC:1.1e-57 


\begin{tabular}{|c|c|c|c|c|}
\hline RC4G0281700 & $\mathrm{rc} 04$ & 39977671 & 39984467 & NBS-LRR \\
\hline RC4G0338200 & $\mathrm{rc} 04$ & 45846444 & 45850390 & NBS \\
\hline RC4G0375900 & $\mathrm{rc} 04$ & 49192413 & 49197402 & CC-NBS-LRR \\
\hline RC4G0479900 & $\mathrm{rc} 04$ & 57383168 & 57385083 & NBS \\
\hline RC4G0480000 & $\mathrm{rc} 04$ & 57386756 & 57392858 & NBS-LRR \\
\hline RC5G0010300 & $\mathrm{rc} 05$ & 599436 & 602538 & NBS \\
\hline RC5G0010900 & $\mathrm{rc} 05$ & 648214 & 651605 & NBS \\
\hline RC5G0030200 & $\mathrm{rc} 05$ & 2012173 & 2016350 & NBS-LRR \\
\hline RC5G0031700 & $\mathrm{rc} 05$ & 2180099 & 2186355 & CC-NBS-LRR \\
\hline RC5G0032000 & $\mathrm{rc} 05$ & 2200115 & 2202139 & NBS-LRR \\
\hline RC5G0059300 & $\mathrm{rc} 05$ & 4052738 & 4053379 & NBS \\
\hline RC5G0061300 & $\mathrm{rc} 05$ & 4199023 & 4201464 & NBS-LRR \\
\hline RC5G0061600 & $\mathrm{rc} 05$ & 4224795 & 4228453 & CC-NBS \\
\hline RC5G0127700 & $\mathrm{rc} 05$ & 9487723 & 9492155 & TIR-NBS-LRR \\
\hline RC5G0128100 & $\mathrm{rc} 05$ & 9506935 & 9509502 & TIR-NBS \\
\hline RC5G0149800 & $\mathrm{rc} 05$ & 11250263 & 11252152 & NBS-LRR \\
\hline RC5G0167000 & $\mathrm{rc} 05$ & 12750787 & 12755732 & NBS-LRR \\
\hline RC5G0169300 & $\mathrm{rc} 05$ & 13053055 & 13055802 & CC-NBS-LRR \\
\hline RC5G0169500 & $\mathrm{rc} 05$ & 13080742 & 13083726 & CC-NBS-LRR \\
\hline RC5G0169800 & $\mathrm{rc} 05$ & 13105656 & 13115300 & NBS-LRR \\
\hline RC5G0227800 & $\mathrm{rc} 05$ & 18936361 & 18942066 & TIR-NBS \\
\hline
\end{tabular}

Apolipopro

tein

P-loop[205-213] Kin-2[280-287] RNBS-B[311-317] GLPL[372-378]

P-loop[1-8] Kin-2[78-85] RNBS-B[106-112] GLPL[171-177]

RPW8

P-loop[201-209] Kin-2[277-284] RNBS-B[306-312] GLPL[362-368] P-loop[83-91] Kin-2[164-171] RNBS-B[191-197] GLPL[252-258]

P-loop[71-79] Kin-2[149-156] RNBS-B[176-182] GLPL[236-242]

P-loop[195-203] Kin-2[270-277] RNBS-B[301-302] GLPL[366-372]

P-loop[185-193] Kin-2[259-266] RNBS-B[287-293] GLPL[351-357]

P-loop[193-201] Kin-2[269-276] RNBS-B[300-306] GLPL[365-371]

P-loop[211-219] Kin-2[284-291] RNBS-B[314-320] GLPL[376-382]

P-loop[102-110] Kin-2[175-182] RNBS-B[205-211] GLPL[267-273]

P-loop[1-8] Kin-2[88-95] RNBS-B[115-121] GLPL[175-181]

P-loop[35-43] Kin-2[124-131] RNBS-B[151-157] GLPL[211-217]

P-loop[181-189] Kin-2[274-281] RNBS-B[301-307] GLPL[361-367]

P-loop[285-293] Kin-2[363-370] RNBS-B[390-396] GLPL[456-462]

P-loop[220-228] Kin-2[298-305] RNBS-B[325-331] GLPL[391-397]

P-loop[23-31] Kin-2[100-107] RNBS-B[124-130] GLPL[184-190]

P-loop[194-202] Kin-2[276-283] RNBS-B[303-309] GLPL[367-373]

P-loop[194-202] Kin-2[277-284] RNBS-B[304-310] GLPL[368-374]

P-loop[194-202] Kin-2[276-283] RNBS-B[303-309] GLPL[367-373]

P-loop[194-202] Kin-2[276-283] RNBS-B[303-309] GLPL[367-373]

P-loop[220-228] Kin-2[298-305] RNBS-B[326-332] GLPL[386-392]
[13-137]Apolipoprotein:0.0038 [177460]NB-ARC:1.8e-57 [910-

944]LRR_5:0.0061 [945-

985]LRR 4:0.00054 [986-

1011]LRR_5:0.0061

[1-268]NB-ARC:1.1e-66

[16-90]RPW8:0.005 [177-451]NB-

ARC:4.1e-52 [568-618]LRR_8:2e-09

[61-332]NB-ARC:1.7e-32

[49-315]NB-ARC:3.4e-36 [600-

658]LRR_8:0.00011 [741-

797]LRR_8:0.00032 [916-

971]LRR_ 8:0.00033

[171-455]NB-ARC:9.2e-65

[162-446]NB-ARC:4.8e-73 [180-451]NB-ARC:7.4e-56 [545-

597]LRR 8:1.9e-05 [885-

945]LRR 8:0.00013

[188-463]NB-ARC:4e-58 [563-

620]LRR_8:1e-05 [792-829]LRR_4:0.0093 [79-354]NB-ARC:2.5e-58 [454478]LRR_8:1.5e-05 [479-517]LRR_4:6.5e-

[1-186]NB-ARC:9.2e-26

[14-308]NB-ARC:1.3e-46 [408-

468]LRR_8:4.4e-09

[161-465]NB-ARC:1.5e-36 [77-247]TIR:5e-46 [253-

279]AAA_16:0.0074 [280-529]NB-

ARC:1.6e-20 [727-762]LRR_4:0.006 [868-

924]LRR_8:0.0037 [985-

1028]LRR 4:0.0002 [1029-

1045]LRR_4:0.0058

[14-192]TIR:6.2e-47 [193-

212]AAA_16:6.1e-05 [213-461]NB-

ARC:3.7e-22 [635-650]LRR_3:0.0014

[4-266]NB-ARC:2e-46 [383-

426]LRR_8:0.0019 [441-

497]LRR_8:0.00096

[172-459]NB-ARC:1.2e-83 [577-

633]LRR_8:6.4e-07

[172-460]NB-ARC:2.6e-80 [579-

632]LRR_8:6.8e-07

[172-459]NB-ARC:2.3e-83 [576-

632]LRR_8:3.2e-06

[173-459]NB-ARC:7.9e-85 [576-

632]LRR_8:7.3e-06 [633-

649]LRR_8:0.00054

[15-194]TIR:1.1e-48 [196-455]NB-

ARC:5.7e-35 [618-634]LRR_3:0.0089 


\begin{tabular}{|c|c|c|c|c|c|}
\hline RC5G0228700 & $\mathrm{rc} 05$ & 19071300 & 19075405 & TIR-NBS-LRR & \\
\hline RC5G0231700 & $\mathrm{rc} 05$ & 19480303 & 19485750 & TIR-NBS & \\
\hline RC5G0245000 & $\mathrm{rc} 05$ & 20532298 & 20535069 & NBS-LRR & \\
\hline RC5G0254700 & $\mathrm{rc} 05$ & 21614563 & 21616935 & TIR-NBS & \\
\hline RC5G0327400 & $\mathrm{rc} 05$ & 30163102 & 30167202 & CC-NBS-LRR & \\
\hline RC5G0343100 & $\mathrm{rc} 05$ & 32481329 & 32483874 & NBS-LRR & \\
\hline RC5G0343400 & $\mathrm{rc} 05$ & 32499168 & 32502401 & CC-NBS-LRR & \\
\hline RC5G0343500 & $\mathrm{rc} 05$ & 32505090 & 32506001 & NBS & \\
\hline RC5G0343700 & $\mathrm{rc} 05$ & 32507785 & 32512256 & CC-NBS-LRR & \\
\hline RC5G0346300 & $\mathrm{rc} 05$ & 32787576 & 32790430 & CC-NBS-LRR & RPW8 \\
\hline RC5G0370200 & $\mathrm{rc} 05$ & 36020158 & 36024058 & CC-NBS-LRR & \\
\hline RC5G0370400 & $\mathrm{rc} 05$ & 36049700 & 36058870 & CC-NBS-LRR & \\
\hline RC5G0370800 & $\mathrm{rc} 05$ & 36124042 & 36132140 & NBS-LRR & \\
\hline RC5G0371100 & $\mathrm{rc} 05$ & 36172573 & 36178226 & CC-NBS-LRR & RPW8 \\
\hline RC5G0376400 & $\mathrm{rc} 05$ & 36979080 & 36981422 & CC-NBS-LRR & RPW8 \\
\hline RC5G0380200 & $\mathrm{rc} 05$ & 37680567 & 37683462 & NBS-LRR & \\
\hline RC5G0403300 & $\mathrm{rc} 05$ & 40299954 & 40303988 & CC-NBS-LRR & \\
\hline RC5G0435500 & $\mathrm{rc} 05$ & 45625860 & 45628523 & NBS & \\
\hline RC5G0441600 & $\mathrm{rc} 05$ & 46313610 & 46317073 & NBS-LRR & \\
\hline RC5G0451400 & $\mathrm{rc} 05$ & 48176707 & 48179831 & NBS-LRR & \\
\hline RC5G0451800 & $\mathrm{rc} 05$ & 48195161 & 48198638 & NBS-LRR & \\
\hline RC5G0452200 & $\mathrm{rc} 05$ & 48274304 & 48277639 & NBS-LRR & \\
\hline RC5G0452700 & $\mathrm{rc} 05$ & 48338371 & 48341154 & NBS-LRR & \\
\hline RC5G0489900 & $\mathrm{rc} 05$ & 55379318 & 55386672 & TIR-NBS-LRR & \\
\hline
\end{tabular}

\section{P-loop[220-228] Kin-2[300-307] RNBS-B[328-334] GLPL[386-392] 745]LRR_8:0.0068}

[15-193]TIR:2.4e-49 [195-459]NBP-loop[219-227] Kin-2[298-305] RNBS-B[326-332] GLPL[384-390] ARC:1.8e-25 [620-635]LRR_3:0.00053 [175-458]NB-ARC:1.4e-65 [600-

P-loop[196-204] Kin-2[274-281] RNBS-B[301-307] GLPL[365-371] 653]LRR_8:1.2e-05

[14-177]TIR:7.4e-51 [200-460]NB-

P-loop[218-226] Kin-2[297-304] RNBS-B[324-330] GLPL[383-389] ARC:3.3e-26

[170-446]NB-ARC:3.4e-65 [538-

595]LRR_8:1e-08 [609-665]LRR_8:1.4e-

P-loop[191-199] Kin-2[269-276] RNBS-B[296-302] GLPL[354-360] 07

[80-358]NB-ARC:1.1e-61 [479-

517]LRR_4:0.00021 [525-

P-loop[106-114] Kin-2[181-188] RNBS-B[210-216] GLPL[271-277] 562]LRR_4:0.0049

[177-454]NB-ARC:9.7e-61 [566-

575]LRR_8:0.0081 [577-

P-loop[203-211] Kin-2[278-285] RNBS-B[307-313] GLPL[368-374] 631]LRR_8:0.00027

P-loop[88-96] Kin-2[165-172] RNBS-B[194-200] GLPL[255-261] [62-289]NB-ARC:1.4e-47

[175-452]NB-ARC:3e-64 [577-

P-loop[201-209] Kin-2[276-283] RNBS-B[305-311] GLPL[366-372] 633]LRR_8:8.8e-06

[4-88]RPW8:0.0071 [177-454]NB-

P-loop[203-211] Kin-2[278-285] RNBS-B[307-313] GLPL[368-374] ARC:8e-66 [575-616]LRR_8:0.00014

[176-453]NB-ARC:7.7e-62 [561-

571]LRR_8:0.0013 [572-

P-loop[202-210] Kin-2[277-284] RNBS-B[306-312] GLPL[367-373] 626]LRR 8:0.00047

[156-436]NB-ARC:1.3e-62 [552-

P-loop[182-190] Kin-2[257-264] RNBS-B[286-292] GLPU347-353] 573]LRR_8:3.2e-06 [574-632]LRR_8:2.4e-

[158-432]NB-ARC:7.7e-64 [553-

P-loop[184-192] Kin-2[259-266] RNBS-B[288-294] GLPL[346-352] $\quad$ 607]LRR_8:2.2e-06

[4-88]RPW8:0.0014 [180-461]NB-

ARC:1.2e-70 [588-626]LRR_4:8.7e-05

P-loop[206-214] Kin-2[281-288] RNBS-B[310-316] GLPL[370-376]

[4-98]RPW8:1.4e-05 [181-433]NB-

P-loop[204-212] Kin-2[270-277] RNBS-B[299-305] GLPL[344-348] ARC:9.7e-43 [556-610]LRR_8:2e-05

[203-212]AAA 16:0.00014 [213-489]NB-

P-loop[234-242] Kin-2[310-317] RNBS-B[340-346] GLPL[401-407] ARC:4.2e-62 [608-641]LRR_4:0.00037 [156-433]NB-ARC:8.7e-63 [536-

P-loop[182-190] Kin-2[257-264] RNBS-B[286-292] GLPL[347-353] 587]LRR_8:1.1e-05

P-loop[19-27] Kin-2[98-105] RNBS-B[125-131] GLPL[182-188]

[5-246]NB-ARC:3.1e-29

[178-464]NB-ARC:1e-56 [586-

P-loop[206-214] Kin-2[281-288] RNBS-B[315-321] GLPL[376-382] 639]LRR_8:0.0015

[172-459]NB-ARC:2e-80 [580-

P-loop[194-202] Kin-2[276-283] RNBS-B[303-309] GLPL[367-373]

P-loop[197-205] Kin-2[279-286] RNBS-B[306-312] GLPL[370-376]

P-loop[194-202] Kin-2[276-283] RNBS-B[303-309] GLPL[367-373]

P-loop[194-202] Kin-2[276-283] RNBS-B[303-309] GLPL[367-373]

P-loop[243-251] Kin-2[322-329] RNBS-B[349-355] GLPL[409-415]
636]LRR $8 \cdot 2.7 \mathrm{e}-06$

[175-461]NB-ARC:1.3e-82 [587-

643]LRR 8:4.5e-07

[172-459]NB-ARC:4.1e-81 [580-

636]LRR_8:8.1e-06

[172-459]NB-ARC:4.1e-82 [580-

636]LRR_8:6.7e- 08

[37-204]TIR:3.7e-48 [221-476]NB- 


\begin{tabular}{|c|c|c|c|c|c|c|c|}
\hline RC5G0490600 & $\mathrm{rc} 05$ & 55468667 & 55471769 & TIR-NBS-LRR & & P-loop[199-207] Kin-2[276-283] RNBS-B[303-309] GLPL[362-368] & $\begin{array}{l}\text { ARC:2.6e-28 [580-598]LRR_3:0.0002 } \\
\text { [672-729]LRR_8:6.1e-06 } \\
\text { [3-95]TIR:7.2e-12 [120-351]NB-ARC:9.2e- } \\
\text { 34 [503-520]LRR_3:0.0035 [756- }\end{array}$ \\
\hline RC5G0491400 & $\mathrm{rc} 05$ & 55585356 & 55590552 & TIR-NBS-LRR & & P-loop[129-137] Kin-2[200-207] RNBS-B[227-233] GLPL[286-292] & $\begin{array}{l}\text { 815]LRR_8:6.2e-05 } \\
\text { [181-463]NB-ARC:1.2e-59 [584- } \\
\text { 605]LRR_8:0.0098 [606- }\end{array}$ \\
\hline RC5G0511300 & $\mathrm{rc} 05$ & 58633590 & 58636220 & CC-NBS-LRR & & P-loop[206-214] Kin-2[281-288] RNBS-B[315-321] GLPL[376-382] & $\begin{array}{l}\text { 645]LRR_4:0.0016 } \\
\text { [2-131]TIR:1.2e-30 [153-429]NB-ARC:6e- } \\
52 \text { [524-580]LRR_8:9.3e-09 [581- }\end{array}$ \\
\hline RC5G0511600 & $\mathrm{rc} 05$ & 58675227 & 58680594 & TIR-NBS-LRR & & P-loop[175-183] Kin-2[250-257] RNBS-B[279-285] GLPL[338-344] & $\begin{array}{l}\text { 591]LRR_4:0.0015 } \\
{[4-255] N B-A R C: 1.1 \mathrm{e}-34[377-}\end{array}$ \\
\hline RC5G0528800 & $\mathrm{rc} 05$ & 61147231 & 61150828 & NBS-LRR & & P-loop[20-28] Kin-2[81-88] RNBS-B[110-116] GLPL[169-175] & $\begin{array}{l}\text { 435]LRR_8:4.4e-08 } \\
\text { [14-187]TIR:1.1e-42 [199-459]NB- }\end{array}$ \\
\hline RC5G0529000 & $\mathrm{rc} 05$ & 61157136 & 61161309 & TIR-NBS-LRR & & P-loop[219-227] Kin-2[280-287] RNBS-B[309-315] GLPL[368-374] & $\begin{array}{l}\text { ARC:2.9e-36 [569-627]LRR_8:9.3e-06 } \\
{[627-637] L R R \_4: 0.00059}\end{array}$ \\
\hline RC5G0529400 & $\mathrm{rc} 05$ & 61185509 & 61186618 & NBS & & P-loop[20-28] Kin-2[81-88] RNBS-B[110-116] GLPL[171-177] & [1-258]NB-ARC:1.5e-36 \\
\hline RC5G0550500 & $\mathrm{rc} 05$ & 64286509 & 64289637 & TIR-NBS-LRR & & P-loop[231-239] Kin-2[306-313] RNBS-B[335-341] GLPL[403-409] & $\begin{array}{l}\text { [22-193]TIR:6.3e-44 [211-489]NB- } \\
\text { ARC:6.2e-48 [589-647]LRR_8:7.7e-10 }\end{array}$ \\
\hline RC5G0550800 & $\mathrm{rc} 05$ & 64342396 & 64347751 & $\begin{array}{l}\text { CC-TIR-NBS- } \\
\text { LRR }\end{array}$ & NAP & P-loop[317-325] Kin-2[391-398] RNBS-B[420-426] GLPL[478-484] & $\begin{array}{l}\text { [101-274]TIR:1e-46 [296-567]NB- } \\
\text { ARC:1.7e-50 [665-724]LRR_8:2.3e-09 } \\
\text { [728-768]LRR_8:0.0006 [1209- } \\
\text { 1450]NAP:1.9e-68 } \\
\text { [2-137]TIR:2.4e-29 [160-352]NB- }\end{array}$ \\
\hline RC5G0551300 & $\mathrm{rc} 05$ & 64363055 & 64364261 & TIR-NBS & & P-loop[179-187] Kin-2[254-261] RNBS-B[283-289] GLPL[342-348] & $\begin{array}{l}\text { ARC:2.8e-34 } \\
{[78-249] T I R: 5.8 \mathrm{e}-46 \text { [267-548]NB- }}\end{array}$ \\
\hline RC5G0551600 & $\mathrm{rc} 05$ & 64416135 & 64418578 & TIR-NBS & & P-loop[288-296] Kin-2[371-378] RNBS-B[400-406] GLPL[459-465] & $\begin{array}{l}\text { ARC:8.1e-46 } \\
{[25-188] \text { TIR:7.8e-44 [212-488]NB- }} \\
\text { ARC:2.2e-46 [586-643]LRR 8:6.3e-09 }\end{array}$ \\
\hline RC5G0551800 & $\mathrm{rc} 05$ & 64429958 & 64436745 & TIR-NBS-LRR & & P-loop[232-240] Kin-2[307-314] RNBS-B[336-342] GLPL[395-401] & $\begin{array}{l}\text { [650-666]LRR_4:0.0053 } \\
{[20-109] \text { TIR:2.8e-10 [121-372]NB- }}\end{array}$ \\
\hline RC5G0555100 & $\mathrm{rc} 05$ & 64897229 & 64898989 & TIR-NBS & & P-loop[134-142] Kin-2[209-216] RNBS-B[236-242] GLPL[295-301] & $\begin{array}{l}\text { ARC:3.2e-34 } \\
\text { [5-100]TIR:9.3e-12 [110-361]NB-ARC:9e- }\end{array}$ \\
\hline RC5G0555900 & rc05 & 65041864 & 65043564 & TIR-NBS & & P-loop[125-133] Kin-2[200-207] RNBS-B[227-233] GLPL[286-292] & $\begin{array}{l}35 \\
{[20-189] \text { TIR:1.4e-46 [213-455]NB- }}\end{array}$ \\
\hline RC5G0569800 & rc05 & 66766343 & 66770979 & TIR-NBS-LRR & & P-loop[227-235] Kin-2[299-306] RNBS-B[326-332] GLPL[385-391] & $\begin{array}{l}\text { ARC:2.6e-35 } \\
\text { [11-181]TIR:1.4e-48 [186- } \\
\text { 208]AAA_16:0.0004 [209-457]NB- } \\
\text { ARC:4e-25 [622-637]LRR_3:3.7e-05 [736- } \\
\text { 789]LRR_8:0.0062 }\end{array}$ \\
\hline RC5G0600200 & $\mathrm{rc05}$ & 69891742 & 69900734 & TIR-NBS-LRR & & P-loop[232-240] Kin-2[307-314] RNBS-B[336-342] GLPL[395-401] & $\begin{array}{l}\text { [23-189]TIR:9.8e-42 [211-479]NB- } \\
\text { ARC:5e-51 [574-632]LRR_8:8.5e-07 [645- } \\
\text { 685]LRR_4:0.00049 [686- } \\
\text { 700]LRR_8:0.0087 }\end{array}$ \\
\hline RC5G0600300 & $\mathrm{rc} 05$ & 69902585 & 69913939 & $\begin{array}{l}\text { CC-TIR-NBS- } \\
\text { LRR }\end{array}$ & & P-loop[233-241] Kin-2[308-315] RNBS-B[337-343] GLPL[396-402] & $\begin{array}{l}\text { [18-190]TIR:1.8e-40 [213-480]NB- } \\
\text { ARC:3.9e-53 [585-643]LRR_8:1.7e-08 } \\
\text { [5-72]TIR:1.6e-06 [101-371]NB-ARC:1.2e- }\end{array}$ \\
\hline RC5G0675300 & $\mathrm{rc} 05$ & 78464769 & 78465971 & TIR-NBS & & P-loop[122-130] Kin-2[197-204] RNBS-B[226-232] GLPL[284-290] & $\begin{array}{l}46 \\
{[10-269] N B-A R C: 6.9 e-52[367-} \\
425] \text { LRR } 8: 2.3 e-12[434-\end{array}$ \\
\hline RC5G0675800 & $\mathrm{rc} 05$ & 78567578 & 78569079 & NBS-LRR & & P-loop[20-28] Kin-2[95-102] RNBS-B[124-130] GLPL[181-187] & 473]LRR_8:0.00049 \\
\hline
\end{tabular}




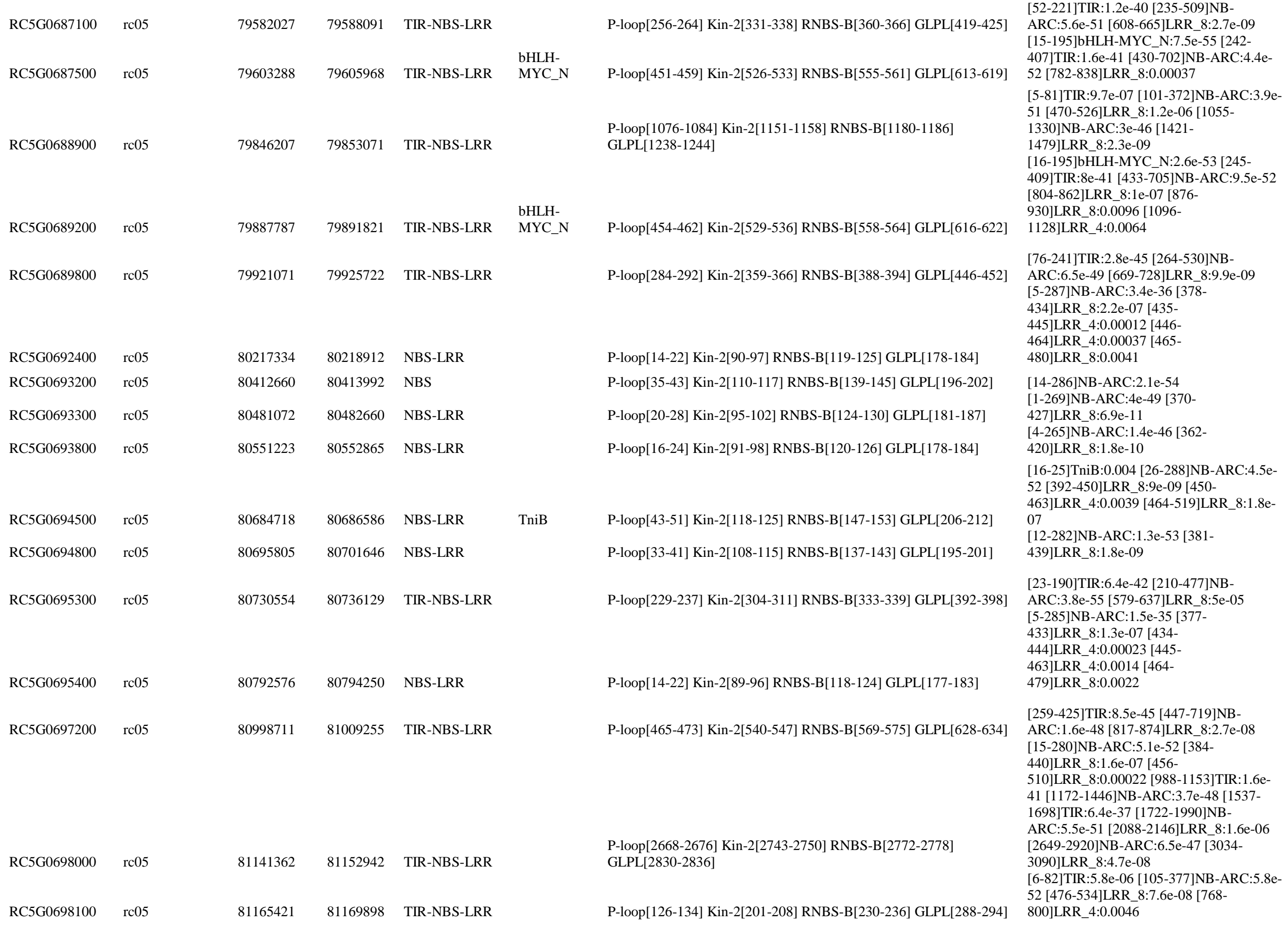




\begin{tabular}{|c|c|c|c|c|}
\hline RC5G0699100 & $\mathrm{rc} 05$ & 81344789 & 81350645 & TIR-NBS-LRR \\
\hline RC5G0699900 & $\mathrm{rc} 05$ & 81493380 & 81497501 & NBS-LRR \\
\hline RC5G0700800 & $\mathrm{rc} 05$ & 81678593 & 81683206 & TIR-NBS-LRR \\
\hline RC5G0700900 & $\mathrm{rc} 05$ & 81694590 & 81695899 & NBS-LRR \\
\hline RC5G0701600 & $\mathrm{rc} 05$ & 81772931 & 81775477 & TIR-NBS-LRR \\
\hline RC5G0703200 & $\mathrm{rc} 05$ & 81915928 & 81920392 & TIR-NBS-LRR \\
\hline RC5G0703900 & $\mathrm{rc} 05$ & 82019665 & 82022336 & TIR-NBS-LRR \\
\hline RC5G0704100 & $\mathrm{rc} 05$ & 82052760 & 82054580 & NBS-LRR \\
\hline RC5G0704400 & $\mathrm{rc} 05$ & 82106576 & 82108154 & NBS-LRR \\
\hline RC5G0707000 & rc05 & 82427759 & 82432941 & TIR-NBS-LRR \\
\hline RC5G0707800 & $\mathrm{rc} 05$ & 82510963 & 82516475 & TIR-NBS-LRR \\
\hline RC5G0716100 & $\mathrm{rc} 05$ & 83403364 & 83404578 & TIR-NBS \\
\hline RC5G0717400 & $\mathrm{rc} 05$ & 83546016 & 83547738 & NBS \\
\hline RC5G0718200 & $\mathrm{rc} 05$ & 83610483 & 83613250 & TIR-NBS \\
\hline RC6G0005500 & $\mathrm{rc} 06$ & 717905 & 722131 & TIR-NBS-LRR \\
\hline RC6G0006000 & rc06 & 738780 & 743774 & TIR-NBS-LRR \\
\hline
\end{tabular}

RC6G0010700 rc06

$1085292 \quad 1090720 \quad$ TIR-NBS-LRR

RC6G0012000 rc06
P-loop[222-230] Kin-2[297-304] RNBS-B[326-332] GLPL[384-390]

P-loop[36-44] Kin-2[111-118] RNBS-B[140-146] GLPL[199-205]

ARC:1.9e-53 [575-633]LRR_8:2.2e-07

[912-948]LRR_4:0.0089

[19-281]NB-ARC:4.7e-51 [385-

443]LRR_8:4.2e-09 [457-512]LRR_8:3.7e07

[17-182]TIR:7.7e-40 [258-420]TIR:4.4e-43 [441-732]NB-ARC:4.6e-39 [824-

879]LRR_8:1.7e-07 [880-

P-loop[461-469] Kin-2[536-543] RNBS-B[565-571] GLPL[624-630] 926]LRR_8:0.00097

[6-266]NB-ARC:6.3e-54 [364-

408]LRR_8:7.3e-05

[68-232]TIR:5.2e-41 [251-524]NB-

P-loop[16-24] Kin-2[91-98] RNBS-B[120-126] GLPL[178-184]

$\begin{array}{ll}\text { P-loop[272-280] Kin-2[347-354] RNBS-B[376-382] GLPL[437-443] } & \text { ARC:9.2e-51 [589-647]LRR_8:1.9e-10 } \\ & {[28-193] \text { TIR:1.3e-38 [262-428]TIR:1.2e-44 }}\end{array}$ [445-734]NB-ARC:5.2e-39 [828-

884]LRR_8:8.9e-08 [885-

895]LRR 4:0.00021 [896-

P-loop[465-473] Kin-2[540-547] RNBS-B[569-575] GLPL[628-634] 905]LRR_4:0.0054

[74-239]TIR:9.8e-43 [258-529]NB-

P-loop[278-286] Kin-2[353-360] RNBS-B[382-388] GLPL[440-446] $\quad$ ARC:3.9e-53 [626-684]LRR_8:2.5e-05

[12-283]NB-ARC:4.9e-57 [381-

438]LRR_8:4.2e-08 [448-

484]LRR_8:0.00025

P-loop[33-41] Kin-2[108-115] RNBS-B[137-143] GLPL[195-201]

P-loop[20-28] Kin-2[95-102] RNBS-B[124-130] GLPL[185-191]

[1-273]NB-ARC:1.6e-50 [372-

430]LRR $8: 2.2 \mathrm{e}-10$

[4-82]TIR:5.8e-06 [107-394]NB-ARC:3.4e-

P-loop[126-134] Kin-2[216-223] RNBS-B[245-251] GLPL[304-310] 38 [492-548]LRR_8:0.00014

[3-251]NB-ARC:5.5e-27 [252-

314]TIR:9.7e-08 [346-516]TIR:5.5e-27

[547-697]TIR:6.5e-26 [734-999]NB-

P-loop[26-34] Kin-2[102-109] RNBS-B[129-135] GLPL[185-191]

ARC:3.8e-49 [1100-1156]LRR_8:1.1e-06 [2-88]TIR:1.5e-15 [104-294]NB-ARC:2.1e-

P-loop[125-133] Kin-2[204-211] RNBS-B[231-237] GLPL[288-294] 21

P-loop[19-27] Kin-2[98-105] RNBS-B[125-131] GLPL[185-191]

[5-251]NB-ARC:7.3e-37

[23-183]TIR:3e-48 [199-454]NB-

P-loop[223-231] Kin-2[302-309] RNBS-B[329-335] GLPL[388-394]

[12-182]TIR:7.5e-50 [204-443]NB-

ARC:4.2e-33 [666-725]LRR_8:0.0021

P-loop[215-223] Kin-2[292-299] RNBS-B[319-325] GLPL[378-384]

7-1053]LRR 8:7.9e-06

[12-182]TIR:2.9e-49 [203-429]NB-

ARC:1.4e-31 [642-682]LRR_4:0.0024

P-loop[215-223] Kin-2[292-299] RNBS-B[319-325] GLPL[378-384] [831-886]LRR_8:2.7e-06

[12-192]TIR:9.5e-43 [198

212]AAA_16:3.3e-05 [213-452]NB-

ARC:7.4e-31 [603-621]LRR_3:3.8e-06

[695-752]LRR 8:8.4e-06 [860-

903]LRR 4:0.00025 [904-

P-loop[222-230] Kin-2[299-306] RNBS-B[326-332] GLPL[385-391] 914]LRR_8:0.0015

[20-181]TIR:3.5e-34 [199-445]NB-

ARC:5.1e-30 [595-613]LRR_3:0.004 [854-

912]LRR_8:0.0001 [912-

P-loop[221-229] Kin-2[293-300] RNBS-B[320-326] GLPL[379-385] 922]LRR_4:0.00053 


\begin{tabular}{|c|c|c|c|c|c|c|c|}
\hline RC6G0019700 & $\mathrm{rc} 06$ & 1964771 & 1968620 & NBS-LRR & & $\begin{array}{l}\text { P-loop[37-45] Kin-2[113-120] RNBS-B[145-151] GLPL[206-212] } \\
\text { P-loop[221-229] Kin-2[298-305] RNBS-B[325-331] GLPL[384-390] }\end{array}$ & $\begin{array}{l}\text { [14-271]NB-ARC:5.9e-39 [437- } \\
\text { 452]LRR_3:0.0035 [577- } \\
\text { 630]LRR_8:0.0052 } \\
\text { [18-188]TIR:2.9e-47 [209-449]NB- } \\
\text { ARC:1.5e-30 }\end{array}$ \\
\hline RC6G0052000 & $\mathrm{rc06}$ & 5413192 & 5415967 & NBS-LRR & & P-loop[27-35] Kin-2[105-112] RNBS-B[132-138] GLPL[191-197] & $\begin{array}{l}\text { [18-262]NB-ARC:7.6e-31 [409- } \\
\text { 427]LRR_3:1.9e-06 [501-557]LRR_8:1.9e- } \\
\text { 05 [646-669]LRR_8:0.0052 [670- } \\
\text { 713]LRR_4:0.00028 }\end{array}$ \\
\hline RC6G0093200 & $\mathrm{rc06}$ & 10156940 & 10162501 & CC-NBS-LRR & RPW8 & P-loop[187-195] Kin-2[264-271] RNBS-B[293-299] GLPL[355-361] & $\begin{array}{l}\text { [22-93]RPW8:0.00035 [166-445]NB- } \\
\text { ARC:6.7e-57 [574-627]LRR_8:3.2e-05 } \\
\text { [153-423]NB-ARC:2.8e-55 [524- } \\
\text { 583]LRR 8:16e-09 [592- }\end{array}$ \\
\hline RC6G0107000 & rc06 & 11477082 & 11479066 & NBS-LRR & & P-loop[172-180] Kin-2[247-254] RNBS-B[279-285] GLPL[338-344] & $\begin{array}{l}\text { 629]LRR_8:0.00025 } \\
\text { [164-444]NB-ARC:1.8e-67 [544- } \\
\text { 601]LRR 8:1.3e-06 [618-670]LRR 8:2.4e- }\end{array}$ \\
\hline RC6G0123900 & $\mathrm{rc06}$ & 13531770 & 13533972 & CC-NBS-LRR & & P-loop[186-194] Kin-2[268-275] RNBS-B[295-301] GLPL[354-360] & $\begin{array}{l}05 \\
{[109-162] Y q e Y: 0.0033 \text { [200-480]NB- }} \\
\text { ARC:1.1e-64 [581-637]LRR_8:5e-09 [655- }\end{array}$ \\
\hline RC6G0124200 & $\mathrm{rc06}$ & 13557623 & 13561228 & CC-NBS-LRR & YqeY & P-loop[222-230] Kin-2[304-311] RNBS-B[331-337] GLPL[390-396] & $\begin{array}{l}\text { 708]LRR_8:0.0081 } \\
\text { [4-79]TIR:1.8e-08 [95-354]NB-ARC:6.4e- }\end{array}$ \\
\hline RC6G0157600 & $\mathrm{rc06}$ & 16429923 & 16432940 & TIR-NBS & & P-loop[117-125] Kin-2[196-203] RNBS-B[223-229] GLPL[283-289] & 34 \\
\hline RC6G0160400 & $\mathrm{rc06}$ & 16755259 & 16771333 & TIR-NBS-LRR & & P-loop[252-260] Kin-2[331-338] RNBS-B[358-364] GLPL[416-422] & $\begin{array}{l}\text { [24-145]TIR:2.9e-40 [243-482]NB- } \\
\text { ARC:1.7e-29 [687-726]LRR_4:0.0036 }\end{array}$ \\
\hline RC6G0160500 & $\mathrm{rc06}$ & 16774763 & 16779151 & TIR-NBS-LRR & & P-loop[217-225] Kin-2[296-303] RNBS-B[323-329] GLPL[382-388] & $\begin{array}{l}\text { [11-177]TIR:6.2e-49 [193-446]NB- } \\
\text { ARC:1.9e-31 [624-657]LRR_4:0.0065 } \\
\text { [720-758]LRR_4:0.0014 [759- } \\
\text { 775]LRR_8:0.0041 } \\
\text { [17-186]TIR:1.6e-48 [198-455]NB- }\end{array}$ \\
\hline RC6G0183500 & rc06 & 20403767 & 20408944 & TIR-NBS & & P-loop[222-230] Kin-2[301-308] RNBS-B[328-334] GLPL[387-393] & ARC:1.9e-23 \\
\hline RC6G0197300 & rc06 & 22624488 & 22628680 & TIR-NBS-LRR & & P-loop[262-270] Kin-2[341-348] RNBS-B[368-374] GLPL[430-436] & $\begin{array}{l}\text { [33-189]TIR:8.8e-48 [250-494]NB- } \\
\text { ARC:1.1e-29 [647-702]LRR_8:0.00061 } \\
\text { [740-763]LRR_8:0.0017 [764- } \\
\text { 820]LRR_8:1.6e-05 } \\
\text { [26-191]TIR:7.1e-43 [212-460]NB- } \\
\text { ARC:4.6e-30 [635-673]LRR_4:0.0007 } \\
\text { [674-697]LRR_4:0.001 [730- } \\
\text { 767]LRR_4:0.0031 [768- } \\
\text { 785]LRR_8:0.0047 }\end{array}$ \\
\hline RC6G0202400 & $\mathrm{rc06}$ & 23439171 & 23442402 & TIR-NBS-LRR & & P-loop[226-234] Kin-2[305-312] RNBS-B[332-338] GLPL[392-398] & $\begin{array}{l}\text { [23-185]TIR:5.6e-45 [204-461]NB- } \\
\text { ARC:6.1e-31 [655-693]LRR_4:0.00058 } \\
\text { [1-250]NB-ARC:3.6e-51 [355- }\end{array}$ \\
\hline RC6G0203300 & rc06 & 23594062 & 23598587 & NBS-LRR & & P-loop[1-8] Kin-2[72-79] RNBS-B[102-108] GLPL[164-170] & $\begin{array}{l}\text { 385]LRR_8:0.00032 [386- } \\
\text { 440]LRR_8:0.00014 } \\
\text { [195-465]NB-ARC:4.1e-60 [586- } \\
\text { 631]LRR_8:0.0013 [632-- } \\
\text { 667]LRR_4:0.0011 [999- } \\
\text { 1056]LRR8:0004 }\end{array}$ \\
\hline RC6G0216200 & $\mathrm{rc06}$ & 25400339 & 25403357 & NBS-LRR & RPW8 & P-loop[181-189] Kin-2[256-263] RNBS-B[285-291] GLPL[347-353] & $\begin{array}{l}\text { [3-58]RPW8:0.0025 [154-434]NB- } \\
\text { ARC:3.5e-63 [556-610]LRR_8:0.0011 }\end{array}$ \\
\hline
\end{tabular}




\begin{tabular}{|c|c|c|c|c|}
\hline RC6G0241800 & $\mathrm{rc} 06$ & 30458368 & 30464847 & TIR-NBS-LRR \\
\hline RC6G0256400 & rc06 & 33268274 & 33273327 & NBS-LRR \\
\hline RC6G0259700 & $\mathrm{rc} 06$ & 33714369 & 33715887 & NBS-LRR \\
\hline RC6G0262100 & $\mathrm{rc} 06$ & 34010916 & 34013831 & CC-NBS-LRR \\
\hline RC6G0265700 & $\mathrm{rc} 06$ & 34555768 & 34566304 & TIR-NBS-LRR \\
\hline RC6G0270900 & $\mathrm{rc} 06$ & 35315757 & 35318664 & TIR-NBS \\
\hline RC6G0271200 & $\mathrm{rc} 06$ & 35365251 & 35371062 & NBS \\
\hline RC6G0276500 & $\mathrm{rc} 06$ & 36143986 & 36148656 & TIR-NBS-LRR \\
\hline RC6G0284500 & $\mathrm{rc} 06$ & 37427969 & 37431126 & CC-NBS-LRR \\
\hline RC6G0290600 & $\mathrm{rc} 06$ & 38547981 & 38551114 & NBS-LRR \\
\hline RC6G0314900 & $\mathrm{rc} 06$ & 41749290 & 41755946 & CC-NBS-LRR \\
\hline RC6G0322100 & $\mathrm{rc} 06$ & 42577765 & 42580401 & TIR-NBS \\
\hline RC6G0323100 & $\mathrm{rc} 06$ & 42663725 & 42665265 & NBS \\
\hline RC6G0323700 & $\mathrm{rc} 06$ & 42727143 & 42728649 & CC-NBS \\
\hline RC6G0362300 & $\mathrm{rc} 06$ & 47266486 & 47268853 & TIR-NBS \\
\hline RC6G0374500 & $\mathrm{rc} 06$ & 48557514 & 48566076 & TIR-NBS \\
\hline RC6G0478700 & $\mathrm{rc} 06$ & 57862436 & 57865361 & NBS \\
\hline RC7G0023600 & $\mathrm{rc} 07$ & 1475070 & 1477290 & NBS-LRR \\
\hline RC7G0033200 & $\mathrm{rc} 07$ & 2131265 & 2134171 & NBS-LRR \\
\hline RC7G0033400 & $\mathrm{rc} 07$ & 2140917 & 2144390 & CC-NBS-LRR \\
\hline
\end{tabular}

[15-195]TIR:3.9e-46 [197-445]NBARC:3.6e-32 [666-685]LRR 3:4.9e-0 [784-806]LRR_4:0.0076 [807846]LRR_4:0.0045 [9971037]LRR_4:0.0066 [10911131]LRR_4:0.0075 [1350-

1405]LRR_8:9.9e-05

[29-194]TIR:2.1e-50 [210221]ATPase_2:0.00028 [222-463]NBARC:1.8e-31 [615-634]LRR_3:0.00016 [755-811]LRR_8:0.0034 [865-

P-loop[233-241] Kin-2[312-319] RNBS-B[339-345] GLPL[398-404] 920]LRR_8:0.0041 P-loop[193-201] Kin-2[275-282] RNBS-B[302-308] GLPL[366-372] $\quad \begin{aligned} & \text { [173-458]NBR_A:0.00027 }\end{aligned}$ [43-317]NB-ARC:3.6e-51 [413470]LRR_8:4.5e-08 [199-464]NB-ARC:1.7e-68 [590P-loop[65-73] Kin-2[140-147] RNBS-B[169-175] GLPL[228-234]

644]LRR 8:2.6e-09

P-loop[210-218] Kin-2[286-293] RNBS-B[315-321] GLPL[377-383

[23-189]TIR:4.2e-49 [209-459]NB-

P-loop[227-235] Kin-2[306-313] RNBS-B[333-339] GLPL[392-398] ARC:2.7e-31 [838-877]LRR 4:4.6e-05 [21-186]TIR:1.4e-50 [203-456]NBP-loop[225-233] Kin-2[305-312] RNBS-B[332-338] GLPL[391-397] ARC:6.1e-31 P-loop[35-43] Kin-2[115-122] RNBS-B[142-148] GLPL[201-207]

[13-266]NB-ARC:1.8e-31

[22-195]TIR:8.5e-47 [202

214]AAA_16:0.0025 [215-462]NBARC:9.4e-37 [620-639]LRR_3:4.4e-05 [665-705]LRR_4:0.0025 [900-

P-loop[230-238] Kin-2[309-316] RNBS-B[336-342] GLPL[395-401] 940]LRR_4:0.0099 [209-498]NB-ARC:1.5e-84 [637693]LRR_8:4.2e-06 [884-

P-loop[232-240] Kin-2[314-321] RNBS-B[341-347] GLPL[405-411] 926]LRR 4:0.0037

[172-459]NB-ARC:1.9e-82 [571627]LRR_8:5e-05 [628-

645]LRR_8:0.00018

P-loop[194-202] Kin-2[276-283] RNBS-B[303-309] GLPL[367-373]

[160-429]NB-ARC:5.2e-50 [607-

P-loop[180-188] Kin-2[254-261] RNBS-B[279-285] GLPL[339-345] 662]LRR_8:6.4e-05

[18-186]TIR:1e-46 [201-454]NB-ARC:2e-

P-loop[222-230] Kin-2[301-308] RNBS-B[328-334] GLPL[388-394] 29 [613-631]LRR_3:0.0022

[1-231]NB-ARC:6.6e-24 [398-

[1-231]NB-ARC:6.6e-24

P-loop[1-8] Kin-2[79-86] RNBS-B[106-112] GLPL[166-172]

[41-303]NB-ARC:8.7e-42

P-loop[60-68] Kin-2[134-141] RNBS-B[161-167] GLPL[220-226]

P-loop[219-227] Kin-2[298-305] RNBS-B[325-331] GLPL[384-390] [16-180]TIR:6.1e-46 [196-447]NBARC:3e-33

[16-185]TIR:3.5e-41 [201-456]NBP-loop[218-226] Kin-2[297-304] RNBS-B[324-330] GLPL[383-389] ARC:5e-33 [610-628]LRR_3:2.2e-05 [9-131]RPW8:2.8e-15 [197-455]NB-

RPW8 P-loop[213-221] Kin-2[290-297] RNBS-B[313-319] GLPL[370-376] C:6.2e-35

[192-465]NB-ARC:1.9e-63 [589-

P-loop[202-210] Kin-2[278-285] RNBS-B[312-318] GLPL[374-380] 644]LRR_8:1.5e-07

[53-67]AAA_16:0.00068 [68-342]NBARC:1.9e-62 [465-519]LRR_8:9.7e-05

P-loop[85-93] Kin-2[159-166] RNBS-B[193-199] GLPL[254-260]

[172-183]AAA_16:0.0022 [184-462]NBP-loop[204-212] Kin-2[278-285] RNBS-B[312-318] GLPL[373-379] ARC:1.7e-64 [584-639]LRR_8:0.00017 


\begin{tabular}{|c|c|c|c|c|}
\hline RC7G0133900 & $\mathrm{rc} 07$ & 10173706 & 10179266 & TIR-NBS-LRR \\
\hline RC7G0225300 & $\mathrm{rc} 07$ & 18963510 & 18972518 & CC-NBS \\
\hline RC7G0235900 & $\mathrm{rc} 07$ & 20055775 & 20061940 & CC-NBS-LRR \\
\hline RC7G0244700 & $\mathrm{rc} 07$ & 21016051 & 21020571 & CC-NBS-LRR \\
\hline RC7G0245500 & $\mathrm{rc} 07$ & 21075666 & 21080081 & NBS-LRR \\
\hline RC7G0252400 & $\mathrm{rc} 07$ & 21761370 & 21763976 & CC-NBS-LRR \\
\hline RC7G0257200 & $\mathrm{rc} 07$ & 22347270 & 22350134 & CC-NBS \\
\hline RC7G0284000 & $\mathrm{rc} 07$ & 25389959 & 25391583 & NBS \\
\hline RC7G0284100 & $\mathrm{rc} 07$ & 25432425 & 25434875 & TIR-NBS \\
\hline RC7G0309000 & $\mathrm{rc} 07$ & 28256648 & 28259491 & NBS-LRR \\
\hline RC7G0319700 & $\mathrm{rc} 07$ & 29485761 & 29488529 & NBS-LRR \\
\hline RC7G0319900 & $\mathrm{rc} 07$ & 29491118 & 29495220 & NBS-LRR \\
\hline RC7G0320000 & $\mathrm{rc} 07$ & 29512402 & 29515306 & NBS-LRR \\
\hline RC7G0320100 & $\mathrm{rc} 07$ & 29517534 & 29521547 & NBS-LRR \\
\hline RC7G0320300 & $\mathrm{rc} 07$ & 29539288 & 29542140 & CC-NBS-LRR \\
\hline RC7G0320600 & $\mathrm{rc} 07$ & 29549108 & 29551951 & NBS \\
\hline RC7G0320900 & $\mathrm{rc} 07$ & 29558456 & 29561305 & CC-NBS-LRR \\
\hline RC7G0321100 & $\mathrm{rc} 07$ & 29566741 & 29569691 & NBS-LRR \\
\hline RC7G0321400 & $\mathrm{rc} 07$ & 29647957 & 29650613 & NBS-LRR \\
\hline RC7G0321800 & $\mathrm{rc} 07$ & 29682960 & 29685842 & CC-NBS-LRR \\
\hline RC7G0321900 & $\mathrm{rc} 07$ & 29688306 & 29691152 & NBS-LRR \\
\hline RC7G0322000 & $\mathrm{rc} 07$ & 29706420 & 29708997 & NBS-LRR \\
\hline RC7G0335300 & $\mathrm{rc} 07$ & 31329692 & 31331125 & NBS \\
\hline RC7G0400300 & $\mathrm{rc} 07$ & 40886140 & 40891483 & CC-TIR-NBS \\
\hline RC7G0416400 & $\mathrm{rc} 07$ & 43459766 & 43467338 & TIR-NBS-LRR \\
\hline
\end{tabular}

[23-187]TIR:1.9e-47 [204-458]NBARC:1e-33 [604-630]LRR_8:0.0025 [631-

P-loop[226-234] Kin-2[305-312] RNBS-B[332-338] GLPL[392-398] 666]LRR_4:0.00099

P-loop[258-266] Kin-2[333-340] RNBS-B[357-363] GLPL[415-421] [238-498]NB-ARC:7.4e-44

[160-416]NB-ARC:2.8e-38 [596-

P-loop[179-187] Kin-2[256-263] RNBS-B[280-286] GLPL[338-344] 652]LRR_8:0.0068

[164-425]NB-ARC:1.2e-44 [608-

P-loop[184-192] Kin-2[260-267] RNBS-B[284-290] GLPL[343-349] 664]LRR_8:9.2e-05

[2-260]NB-ARC:1.1e-42 [436-

P-loop[19-27] Kin-2[95-102] RNBS-B[119-125] GLPL[178-184] 492]LRR_8:0.00016

[221-481]NB-ARC:4e-44 [657-

P-loop[242-250] Kin-2[316-323] RNBS-B[340-346] GLPL[399-405] 713]LRR_8:0.00017

P-loop[204-212] Kin-2[279-286] RNBS-B[310-316] GLPL[371-377] [187-460]NB-ARC:1.4e-59

P-loop[23-31] Kin-2[101-108] RNBS-B[128-134] GLPL[188-194]

[14-259]NB-ARC:1.4e-32

[46-213]TIR:5.4e-46 [228-491]NB-

P-loop[251-259] Kin-2[330-337] RNBS-B[357-363] GLPL[417-423] ARC:4.1e-37

[172-461]NB-ARC:1.1e-74 [576-

598]LRR_8:0.0033 [599-

P-loop[195-203] Kin-2[277-284] RNBS-B[304-310] GLPL[366-372] 649]LRR_8:0.00046

P-loop[199-207] Kin-2[281-288] RNBS-B[308-314] GLPL[370-376]

P-loop[199-207] Kin-2[281-288] RNBS-B[308-314] GLPL[370-376]

[177-463]NB-ARC:1.3e-76 [176-465]NB-ARC:2.8e-77 [605655]LRR_8:0.0027

[173-462]NB-ARC:6.3e-76 [600-

P-loop[196-204] Kin-2[278-285] RNBS-B[305-311] GLPL[367-373] 650]LRR_8:0.0027

[175-462]NB-ARC:1.8e-75 [600-

P-loop[196-204] Kin-2[278-285] RNBS-B[305-311] GLPL[367-373] 650]LRR_8:0.0015

[171-458]NB-ARC:7.7e-75 [579-

634]LRR_8:0.00019 [635-

P-loop[192-200] Kin-2[276-283] RNBS-B[303-309] GLPL[365-371] 652]LRR_8:0.0016

P-loop[192-200] Kin-2[274-281] RNBS-B[301-307] GLPL[363-369] [170-456]NB-ARC:4.6e-70

[171-458]NB-ARC:1.6e-75 [601-

P-loop[192-200] Kin-2[276-283] RNBS-B[303-309] GLPL[365-371] 651]LRR_8:0.0043

[100-386]NB-ARC:6.6e-74 [507-

562]LRR 8:9.1e-05 [563-

P-loop[121-129] Kin-2[205-212] RNBS-B[232-238] GLPL[294-300] 580]LRR_8:0.0014

[100-387]NB-ARC:2.6e-71 [507-

562]LRR_8:4.6e-05 [563-

P-loop[121-129] Kin-2[205-212] RNBS-B[232-238] GLPL[294-300] $\quad$ 580]LRR_8:0.0011

[190-463]NB-ARC:3.3e-72 [583-

638]LRR_8:2.3e-05 [639-

P-loop[198-206] Kin-2[282-289] RNBS-B[309-315] GLPL[370-376] 656]LRR_8:0.00045

[170-458]NB-ARC:1.9e-75 [577-

632]LRR_8:7.3e-06 [633-

P-loop[191-199] Kin-2[275-282] RNBS-B[302-308] GLPL[364-370] 650]LRR_8:0.0011

[81-347]NB-ARC:4.6e-49 [449-

508]LRR_8:6.9e-10 [509-

P-loop[101-109] Kin-2[176-183] RNBS-B[205-211] GLPL[263-269] 518]LRR_4:0.0021

P-loop[186-194] Kin-2[265-272] RNBS-B[292-298] GLPL[353-359] [165-441]NB-ARC:2.1e-75 [17-188]TIR:5.6e-47 [209-479]NB-

P-loop[230-238] Kin-2[305-312] RNBS-B[334-340] GLPL[393-399] ARC:8.3e-51

[22-194]TIR:2.1e-51 [204-454]NBARC:1.5e-31 [607-626]LRR_3:0.00018 [864-885]LRR_4:0.0079 [889-

P-loop[226-234] Kin-2[304-311] RNBS-B[330-336] GLPL[389-395]９28]LRR_4:8.8e-05 


\begin{tabular}{|c|c|c|c|c|}
\hline RC7G0421200 & $\mathrm{rc} 07$ & 44042650 & 44045331 & NBS-LRR \\
\hline RC7G0421300 & $\mathrm{rc} 07$ & 44047157 & 44048711 & TIR-NBS \\
\hline RC7G0429300 & $\mathrm{rc} 07$ & 45382761 & 45384998 & NBS-LRR \\
\hline RC7G0429700 & $\mathrm{rc} 07$ & 45486721 & 45489588 & CC-NBS-LRR \\
\hline RC7G0429900 & $\mathrm{rc} 07$ & 45552832 & 45555696 & CC-NBS-LRR \\
\hline RC7G0452100 & $\mathrm{rc} 07$ & 49220915 & 49227770 & $\begin{array}{l}\text { CC-TIR-NBS- } \\
\text { LRR }\end{array}$ \\
\hline RC7G0452700 & $\mathrm{rc} 07$ & 49282300 & 49289216 & TIR-NBS-LRR \\
\hline RC7G0454900 & $\mathrm{rc} 07$ & 49474139 & 49476752 & CC-NBS-LRR \\
\hline RC7G0460600 & $\mathrm{rc} 07$ & 50106007 & 50108856 & CC-NBS-LRR \\
\hline RC7G0461100 & $\mathrm{rc} 07$ & 50173383 & 50178731 & CC-NBS-LRR \\
\hline RC7G0463700 & $\mathrm{rc} 07$ & 50685389 & 50689356 & NBS-LRR \\
\hline RC7G0463900 & $\mathrm{rc} 07$ & 50732972 & 50735776 & NBS-LRR \\
\hline RC7G0464500 & $\mathrm{rc} 07$ & 50812770 & 50814191 & CC-NBS \\
\hline RC7G0465100 & $\mathrm{rc} 07$ & 50857572 & 50862536 & CC-NBS-LRR \\
\hline RC7G0465900 & $\mathrm{rc} 07$ & 50945167 & 50948053 & CC-NBS-LRR \\
\hline RC7G0466400 & $\mathrm{rc} 07$ & 50969677 & 50971132 & NBS \\
\hline RC7G0466700 & $\mathrm{rc} 07$ & 50980655 & 50990938 & CC-NBS-LRR \\
\hline RC7G0467400 & $\mathrm{rc} 07$ & 51045826 & 51049263 & CC-NBS-LRR \\
\hline RC7G0468200 & $\mathrm{rc} 07$ & 51139885 & 51140885 & NBS \\
\hline RC7G0468400 & $\mathrm{rc} 07$ & 51151640 & 51153007 & CC-NBS \\
\hline
\end{tabular}

P-loop[16-24] Kin-2[91-98] RNBS-B[120-126] GLPL[179-185]

P-loop[171-179] Kin-2[246-253] RNBS-B[275-281] GLPL[333-339]

P-loop[1-8] Kin-2[75-82] RNBS-B[106-112] GLPL[168-174]

DUF3656

P-loop[210-218] Kin-2[285-292] RNBS-B[316-322] GLPL[378-384]

P-loop[210-218] Kin-2[285-292] RNBS-B[316-322] GLPL[378-384]

-loop[232-240] Kin-2[308-315] RNBS-B[335-341] GLPL[394-400]

P-loop[222-230] Kin-2[299-306] RNBS-B[330-336] GLPL[392-398]

P-loop[211-219] Kin-2[286-293] RNBS-B[317-323] GLPL[379-385]

P-loop[210-218] Kin-2[285-292] RNBS-B[317-323] GLPL[379-385]

P-loop[64-72] Kin-2[139-146] RNBS-B[170-176] GLPL[232-238]

DUF3656

DUF3656

P-loop[205-213] Kin-2[280-287] RNBS-B[311-317] GLPL[373-379

P-loop[205-213] Kin-2[282-289] RNBS-B[313-319] GLPL[375-381]

P-loop[214-222] Kin-2[289-296] RNBS-B[320-326] GLPL[382-388]

P-loop[218-226] Kin-2[295-302] RNBS-B[326-332] GLPL[388-394]

P-loop[184-192] Kin-2[266-273] RNBS-B[294-300] GLPL[360-366]

P-loop[215-223] Kin-2[290-297] RNBS-B[321-327] GLPL[383-389]

P-loop[222-230] Kin-2[297-304] RNBS-B[328-334] GLPL[390-396] P-loop[96-104] Kin-2[178-185] RNBS-B[206-212] GLPL[272-278]

DUF3656
[6-261]NB-ARC:1.8e-46 [368426]LRR_8:7.3e-08 [459-

495]LRR_8:0.00084

[1-135]TIR:2.5e-32 [152-418]NB-

ARC:3.3e-49

[1-257]NB-ARC:4.3e-59 [348-

388]LRR_8:1.9e-05 [401-

436]LRR_8:0.0072

[15-79]DUF3656:0.0047 [185-467]NB-

ARC:5.8e-63 [558-598]LRR_8:2.6e-05

[611-646]LRR_8:0.0096

[183-467]NB-ARC:5.4e-62 [558-

598]LRR 8:4.7e-06

[27-195]TIR:2.8e-47 [209-464]NB-

ARC:3.1e-36 [641-696]LRR_8:0.00042

[716-742]LRR_8:0.00015 [743-

783]LRR_4:0.00012

[27-186]TIR:2.9e-48 [209-461]NBARC:1.9e-32 [612-668]LRR_8:0.00036 [754-809]LRR_8:0.00045 [825-

[213-482]NB-ARC:2.3e-63 [571-

613]LRR_8:3.2e-06 [614-

656]LRR_8:0.00019

[180-191]AAA_16:0.0042 [192-464]NB-

ARC:6e-61 [595-649]LRR_8:4.7e-07

[179-190]AAA_16:0.0083 [191-464]NB-

ARC:1.8e-61 [596-651]LRR_8:1.1e-06

[36-319]NB-ARC:1.7e-61 [410-

453]LRR 8:1.4e-06 [454-496]LRR_8:1.7e06

[24-79]DUF3656:0.0054 [177-462]NB-

ARC:9e-61 [557-598]LRR_8:9.7e-06

[20-79]DUF3656:0.0027 [180-460]NB-

ARC:2.5e-58

[183-192]AAA_16:0.00042 [193-470]NB-

ARC: $1.6 \mathrm{e}-63$ [561-604]LRR $8: 2.3 \mathrm{e}-07$

[605-650]LRR_8:4.3e-06

[193-474]NB-ARC:2.1e-57 [567-

608]LRR_8:1.2e-06 [625-

659]LRR_8:0.0015

[174-374]NB-ARC:3.8e-25 [189-470]NB-ARC:1.3e-60 [557599]LRR_8:1.9e-07 [607-

650]LRR_8:0.0032

[185-216]AAA_16:0.0077 [214-477]NBARC:4.3e-61 [571-603]LRR 8:0.00031

[604-659]LRR_8:0.00027

[63-84]AAA_16:0.00016 [86-286]NB-

ARC:2.1e-27

[21-79]DUF3656:0.0031 [179-454]NB-

ARC:3.7e-60 


\begin{tabular}{|c|c|c|c|c|c|}
\hline RC7G0469000 & $\mathrm{rc} 07$ & 51206574 & 51209390 & CC-NBS-LRR & DUF3656 \\
\hline RC7G0469600 & $\mathrm{rc} 07$ & 51262264 & 51265140 & CC-NBS-LRR & \\
\hline RC7G0470500 & $\mathrm{rc} 07$ & 51326238 & 51328673 & CC-NBS-LRR & DUF3656 \\
\hline RC7G0470700 & $\mathrm{rc} 07$ & 51341530 & 51343590 & CC-NBS-LRR & \\
\hline RC7G0471000 & $\mathrm{rc} 07$ & 51430939 & 51431977 & CC-NBS & \\
\hline RC7G0471600 & $\mathrm{rc} 07$ & 51471327 & 51473867 & NBS-LRR & \\
\hline RC7G0475200 & $\mathrm{rc} 07$ & 51830046 & 51831773 & CC-NBS & DUF3656 \\
\hline RC7G0484900 & $\mathrm{rc} 07$ & 53080293 & 53083711 & CC-NBS-LRR & \\
\hline RC7G0486200 & $\mathrm{rc} 07$ & 53255434 & 53258352 & CC-NBS-LRR & \\
\hline RC7G0486400 & $\mathrm{rc} 07$ & 53319282 & 53321738 & CC-NBS-LRR & \\
\hline RC7G0486600 & $\mathrm{rc} 07$ & 53337335 & 53338855 & NBS-LRR & \\
\hline RC7G0486900 & $\mathrm{rc} 07$ & 53398628 & 53401504 & CC-NBS-LRR & \\
\hline RC7G0487800 & $\mathrm{rc} 07$ & 53556508 & 53558706 & CC-NBS-LRR & \\
\hline RC7G0488000 & $\mathrm{rc} 07$ & 53612846 & 53615618 & CC-NBS-LRR & \\
\hline RC7G0488200 & $\mathrm{rc} 07$ & 53658690 & 53661166 & CC-NBS-LRR & \\
\hline RC7G0488400 & $\mathrm{rc} 07$ & 53703331 & 53706225 & CC-NBS-LRR & \\
\hline RC7G0488900 & $\mathrm{rc} 07$ & 53790542 & 53792110 & CC-NBS-LRR & \\
\hline RC7G0497000 & $\mathrm{rc} 07$ & 54968789 & 54971929 & NBS-LRR & \\
\hline RC7G0497100 & $\mathrm{rc} 07$ & 55007186 & 55010884 & CC-NBS-LRR & RPW8 \\
\hline RC7G0507600 & $\mathrm{rc} 07$ & 56734367 & 56738253 & TIR-NBS-LRR & \\
\hline RC7G0508000 & $\mathrm{rc} 07$ & 56794636 & 56797637 & TIR-NBS-LRR & \\
\hline
\end{tabular}

[19-79]DUF3656:0.005 [179-463]NBARC:8.6e-62 [560-615]LRR_8:2.6e-07 [616-640]LRR_8:8.9e-05 [186-469]NB-ARC:1.3e-63 [564603]LRR_8:5.8e-06 [616-

P-loop[214-222] Kin-2[289-296] RNBS-B[320-326] GLPL[382-388] 652]LRR_8:0.00051

[19-79]DUF3656:0.0072 [179-462]NBARC:7.9e-63 [554-595]LRR_8:2.1e-05 $\begin{array}{ll} & \text { ARC:7.9e-63 [554-595]LR } \\ \text { P-loop[204-212] Kin-2[282-289] RNBS-B[313-319] GLPL[375-381] } & \text { [603-647]LRR_8:0.0022 }\end{array}$ [186-469]NB-ARC:1.1e-61 [561602]LRR_8:8.7e-07 [613-

P-loop[214-222] Kin-2[289-296] RNBS-B[320-326] GLPL[382-388] 654]LRR_8:0.003

[63-84]AAA_16:0.00031 [86-286]NBP-loop[96-104] Kin-2[178-185] RNBS-B[206-212] GLPL[272-278] ARC: $8.4 \mathrm{e}-26$ $\begin{array}{ll}\text { P-loop[102-110] Kin-2[177-184] RNBS-B[208-214] GLPL[270-276] } & \text { 521]LRR_8:2.7e-09 }\end{array}$ [22-79]DUF3656:0.0083 [173204]AAA_16:0.0042 [201-467]NB-

P-loop[210-218] Kin-2[287-294] RNBS-B[318-324] GLPL[380-386] ARC:1.4e-63 [184-467]NB-ARC:4e-61 [561-
603]LRR_8:6.7e-06 [604-649]LRR_8:6.7e-

P-loop[210-218] Kin-2[287-294] RNBS-B[318-324] GLPL[380-386] [185-477]NB-ARC:7.7e-58 [587-

P-loop[207-215] Kin-2[295-302] RNBS-B[326-332] GLPL[389-395] 635]LRR_8:2.7e-09

[184-466]NB-ARC:4.8e-60 [576623]LRR_8:1.7e-08 [631-

P-loop[208-216] Kin-2[284-291] RNBS-B[315-321] GLPL[378-384] 655]LRR_4:0.0057

[31-308]NB-ARC:1.2e-57 [423472]LRR_8:1.4e-08 [480-

497]LRR_8:0.00047

P-loop[55-63] Kin-2[131-138] RNBS-B[162-168] GLPL[225-231]

[184-466]NB-ARC:4.7e-57 [577-

P-loop[208-216] Kin-2[285-292] RNBS-B[316-322] GLPL[379-385] 626]LRR_8:1.2e-07

[183-465]NB-ARC:1.9e-61 [575-

P-loop[207-215] Kin-2[283-290] RNBS-B[314-320] GLPL[377-383]

623]LRR 8:3e-09

P-loop[209-217] Kin-2[304-311] RNBS-B[335-341] GLPL[397-403]

[185-484]NB-ARC:8.8e-59 [595-

$\begin{array}{ll}\text { [185-472]NB-ARC:4.5e-59 [582- } & \text { 598]LRR_8:6e-07 [599-654]LRR_8:5.2e- }\end{array}$

P-loop[209-217] Kin-2[290-297] RNBS-B[321-327] GLPL[384-390] 08

[183-466]NB-ARC:2e-61 [576625]LRR_8:5.3e-07 [626-

P-loop[207-215] Kin-2[284-291] RNBS-B[315-321] GLPL[378-384] 654]LRR_4:0.0031

[1-262]NB-ARC:9.7e-57 [373-

422]LRR_8:1.1e-10 [423-

446]LRR_4:0.00021

P-loop[1-8] Kin-2[81-88] RNBS-B[112-118] GLPL[175-181]

[35-314]NB-ARC:5.4e-60 [440-

495]LRR_8:1.9e-06 [495-

P-loop[62-70] Kin-2[137-144] RNBS-B[166-172] GLPL[227-233]

519]LRR_4:0.00038

[4-95]RPW8:0.0029 [223-502]NB-

ARC:1.7e-65 [625-680]LRR_8:9.4e-05

P-loop[250-258] Kin-2[325-332] RNBS-B[354-360] GLPL[415-421] [680-709]LRR_4:0.00049

[13-177]TIR:4.4e-47 [191-446]NB-

P-loop[214-222] Kin-2[293-300] RNBS-B[320-326] GLPL[379-385] ARC:5.8e-32 [643-701]LRR_8:0.00076

[1-84]TIR:5.7e-13 [104-359]NB-ARC:2.1e-

P-loop[128-136] Kin-2[207-214] RNBS-B[234-240] GLPL[292-298] 37 [512-567]LRR_8:0.00046 


$\begin{array}{lllll}\text { RC7G0511800 } & \mathrm{rc07} & 57337077 & 57340131 & \text { TIR-NBS-LRR } \\ \text { RC7G0527800 } & \mathrm{rc07} & 59327412 & 59329960 & \text { CC-NBS } \\ \text { RC7G0530500 } & \mathrm{rc07} & 59779621 & 59782561 & \text { NBS-LRR } \\ & & & & \\ \text { RC7G0567300 } & \mathrm{rc07} & 65553075 & 65558854 & \text { TIR-NBS-LRR } \\ & & & & \\ \text { RC7G0567700 } & \mathrm{rc07} & 65614421 & 65619817 & \text { NBS-LRR }\end{array}$

P-loop[252-260] Kin-2[331-338] RNBS-B[358-364] GLPL[417-423] P-loop[179-187] Kin-2[255-262] RNBS-B[279-285] GLPL[338-344]

P-loop[197-205] Kin-2[272-279] RNBS-B[302-308] GLPL[363-369

P-loop[299-307] Kin-2[376-383] RNBS-B[402-408] GLPL[462-468]

P-loop[26-34] Kin-2[103-110] RNBS-B[129-135] GLPL[189-195]
[48-210]TIR:2.7e-45 [228-482]NB-

ARC:1.9e-32 [652-690]LRR_8:0.00054

[160-422]NB-ARC:5.7e-44

[169-450]NB-ARC:1.3e-62 [581-

635]LRR_8:2.3e-06

[96-267]TIR:7.1e-49 [276-535]NB-

ARC:1.2e-37 [681-700]LRR 3:0.0004

[926-984]LRR_8:0.00057

[3-259]NB-ARC:1.9e-37 [500-

523]LRR_8:0.0047 [524-

580]LRR_8:0.0003 [642-

700]LRR_8:0.00065 


\section{Supplementary figures}

1097 Supp. Fig. 1 Evaluation zone and rating scale of black spot disease in rose

1098 From 0 (no symptoms) to 5 (total defoliation of the plant) with a score of 1 for less than $25 \%$ of infected leaflets, a

1099 score of 2 for infection between 25 and 50\%, a score of 3 for infection between 50 and $75 \%$ of infected leaflets and 4

1100 for infection of $75 \%$ to $100 \%$ and partial defoliation.

1101

1102 


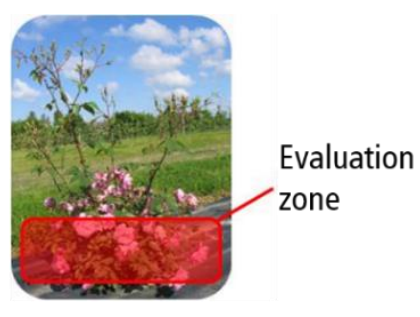

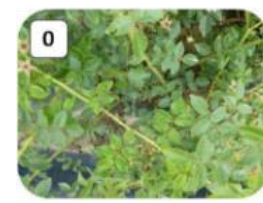

Absence

of

symptoms

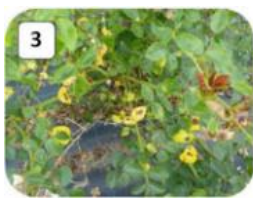

$50<x \leq 75 \%$ of infected leaflets

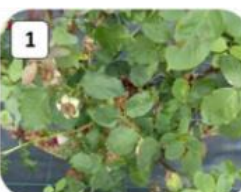

$0<x \leq 25 \%$ of infected leaflets

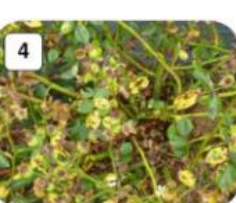

$75<x \leq 100 \%$ of infected leaflets

+ Partial

defoliation

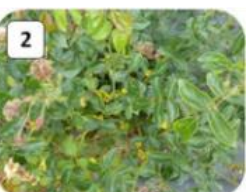

$25<x \leq 50 \%$ of infected leaflets

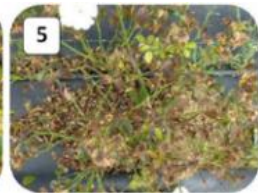

Total defoliation 
1103 Supp. Fig. 2 Overall mean of black spot disease (BSD) scores for three rose populations over several locations and

1104 years under natural infections in field

1105 In the box plots, the boundaries of the box indicate the 25 th percentile (on the lower part) and the 75 th percentile (on

1106 the upper part). A thick line within the box marks the median, and a dot within the box marks the mean. Lines above

1107 and below the box indicate the 10th and 90th percentiles. Points above and below the whiskers indicate outliers outside

1108 the 10th and 90th percentiles. A: Data description for different years for OW population in Angers, B: Data description

1109 for different years for FW population in Angers, C: Data description for different years for HW population in Angers,

1110 Bellegarde and Diémoz. A Kruskal-Wallis test was used to compare the scoring years for HW and OW populations,

1111 and a Wilcoxon test was used to compare the two scoring years 2014-2018 for FW. For HW population, locations were

1112 considered separately. P-values of the tests are displayed on the graphs for all populations.

1113

1114 
1115 Supp. Fig. 3 Genetic linkage map of the male parent hybrid, Rosa wichurana, for OW population

1116 Linkage groups (LG) names for the male map (B1 to B7) are placed above the corresponding linkage groups according

1117 to Spiller et al. 2011. Locus names are indicated on the right side of each LG. When several markers were mapped at

1118 a same position, one or two markers were reported corresponding to the unique loci or markers with different phases.

1119 SSR markers are indicated in red. Genetic distances (cM) are indicated on the left side of each LG.

1120

1121 

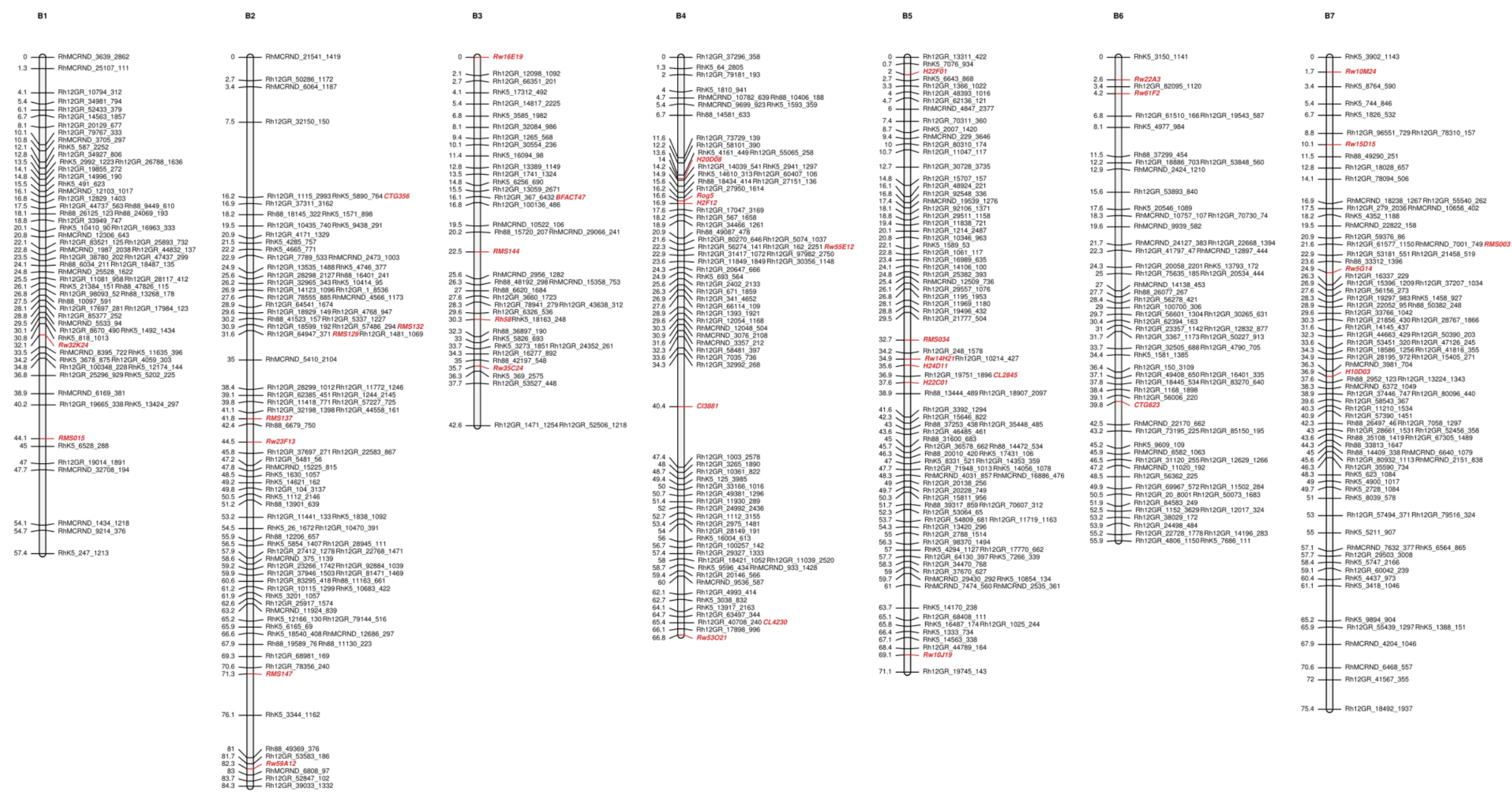

Supplementary figure 3: Genetic linkage map of the male parent hybrid, Rosa wichurana, for OW population 
1122 Supp. Fig. 4 Genetic linkage map of the female parent, Rosa chinensis 'Old Blush', for OW population

1123 Linkage groups (LG) names for the female map (A1 to A7) are placed above the corresponding linkage groups. Locus

1124 names are indicated on the right side of each LG. When several markers were mapped at the same position, one or two

1125 markers were reported corresponding to the unique loci or markers with different phases. SSR markers are indicated

1126 in red. Genetic distances (cM) are indicated on the left side of each LG.

1127

1128 
A1

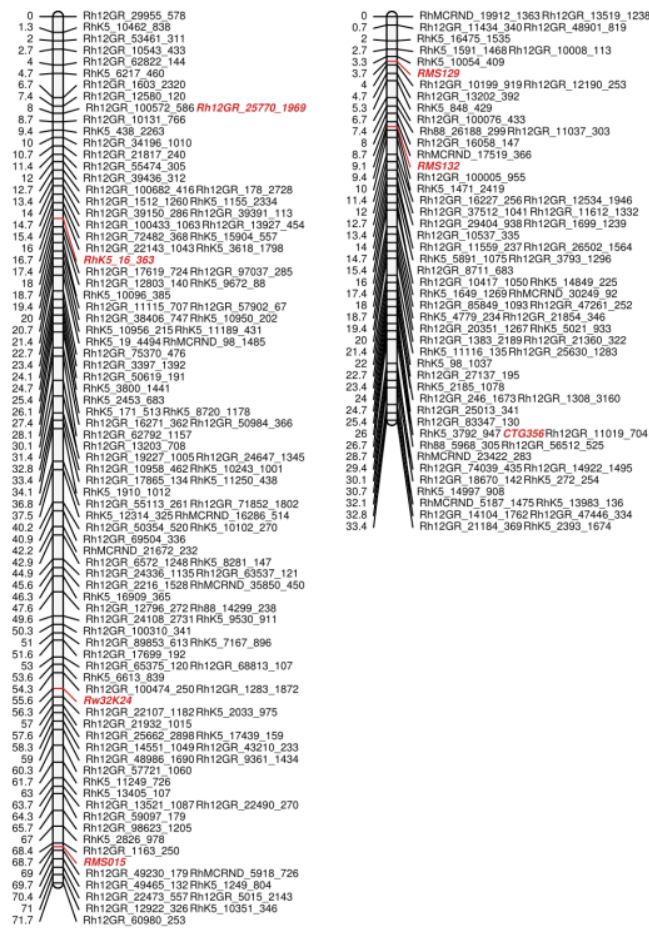

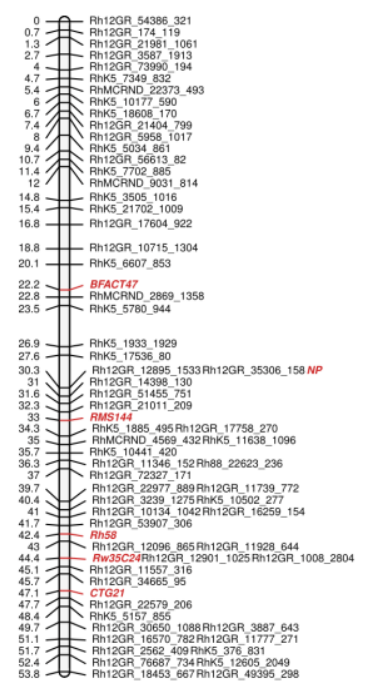

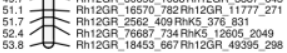

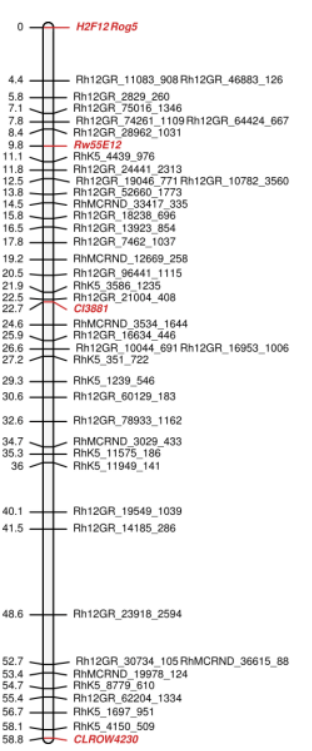

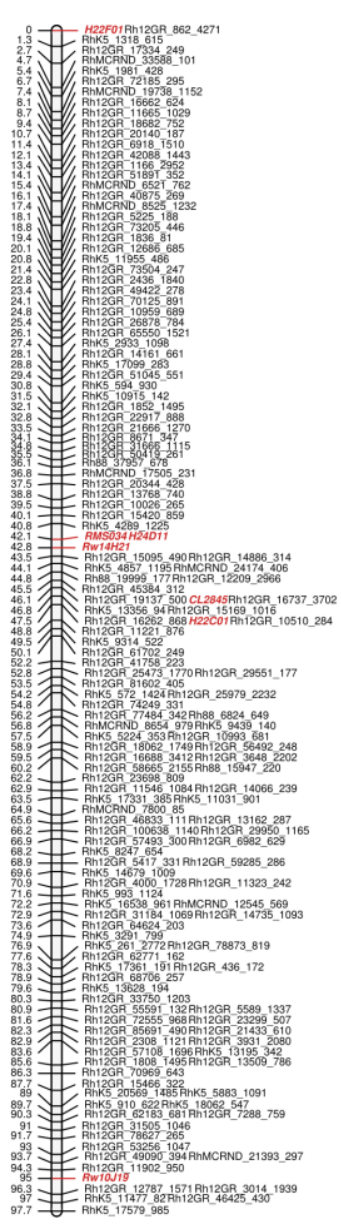
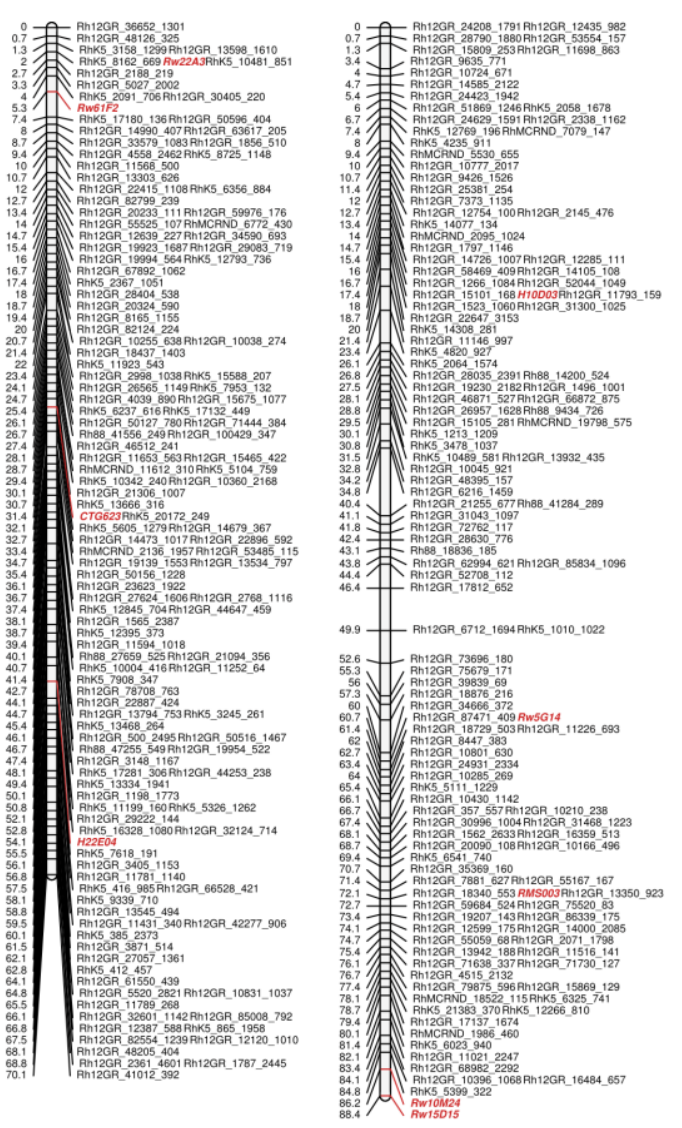

Supplementary figure 4: Genetic linkage map of the female parent, Rosa chinensis 'Old Blush', for OW population 
1129 Supp. Fig. 5 QTL mapping associated with black spot disease (BSD) resistance using a normal model with an CIM

1130 analysis for OW population for the male map

1131 Linkage groups are named as follows: "B" for the male map and the number of the linkage group. LOD score for each

1132 year and the mean of all years are calculated using a Composite Interval Mapping method (CIM) and are displayed

1133 with different colors. The same set of colors is used to represent the $\alpha=0.05$ LOD threshold for declaring significant

1134 QTL based on 1,000 permutations.

1135

1136 


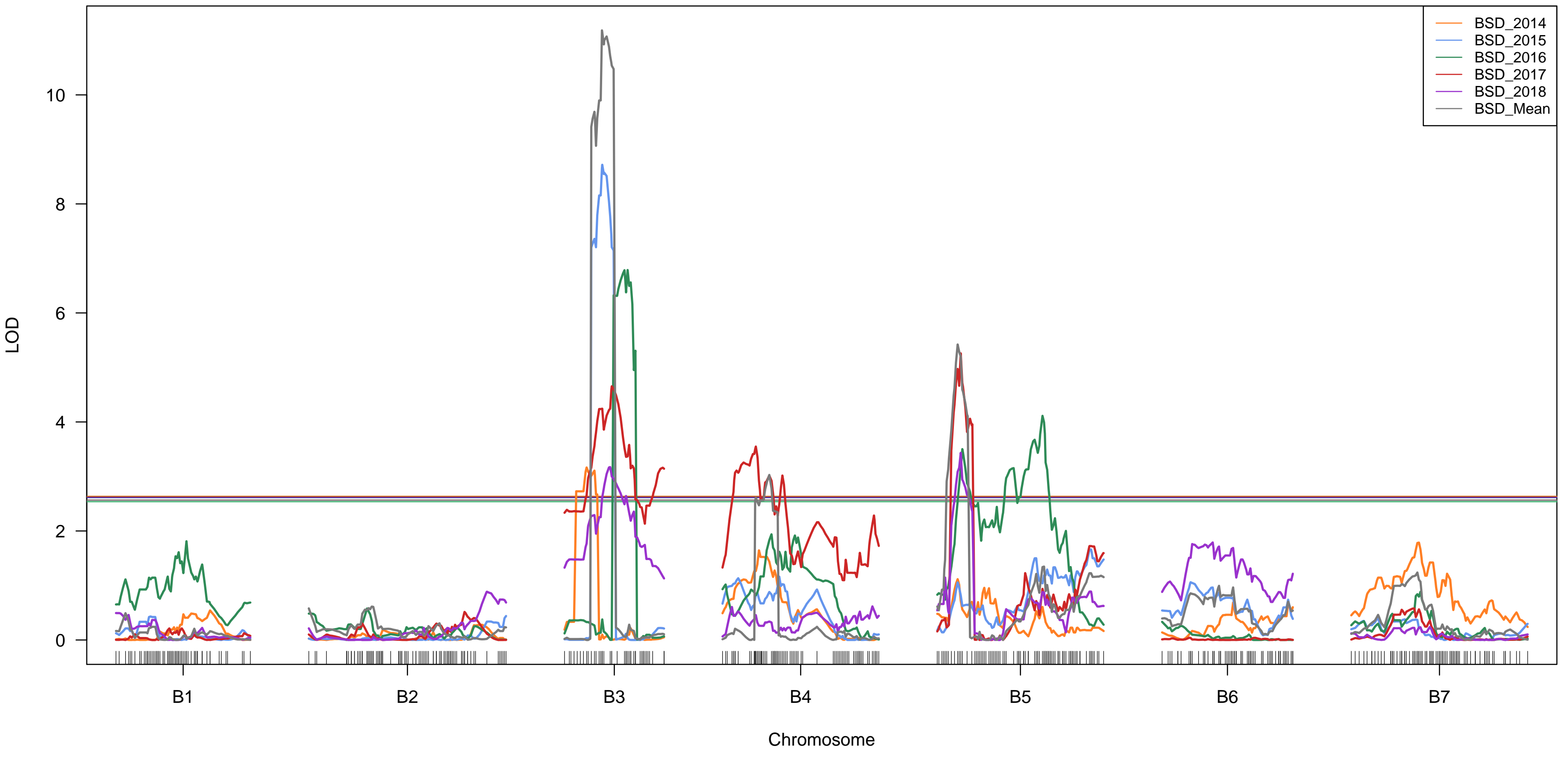


1137 Supp. Fig. 6 QTL mapping associated with black spot disease resistance using a two-part model approach for non-

1138 normally distributed data of FW population for the male map

1139 A and B: LOD curves for the two-part model for 2014 (A) and 2018 (B); LOD. $\pi$ (penetrance, equivalent to binary

1140 model) displayed in blue, LOD. $\mu$ (severity, equivalent to normal model for non-spike phenotypes) displayed in green

1141 and LOD. $\pi . \mu$ (sum, representing the complete model) displayed in black; LOD thresholds are displayed in red with

$1142 \alpha=0.05$ for declaring significant QTL based on 1,000 permutations.

1143

1144 


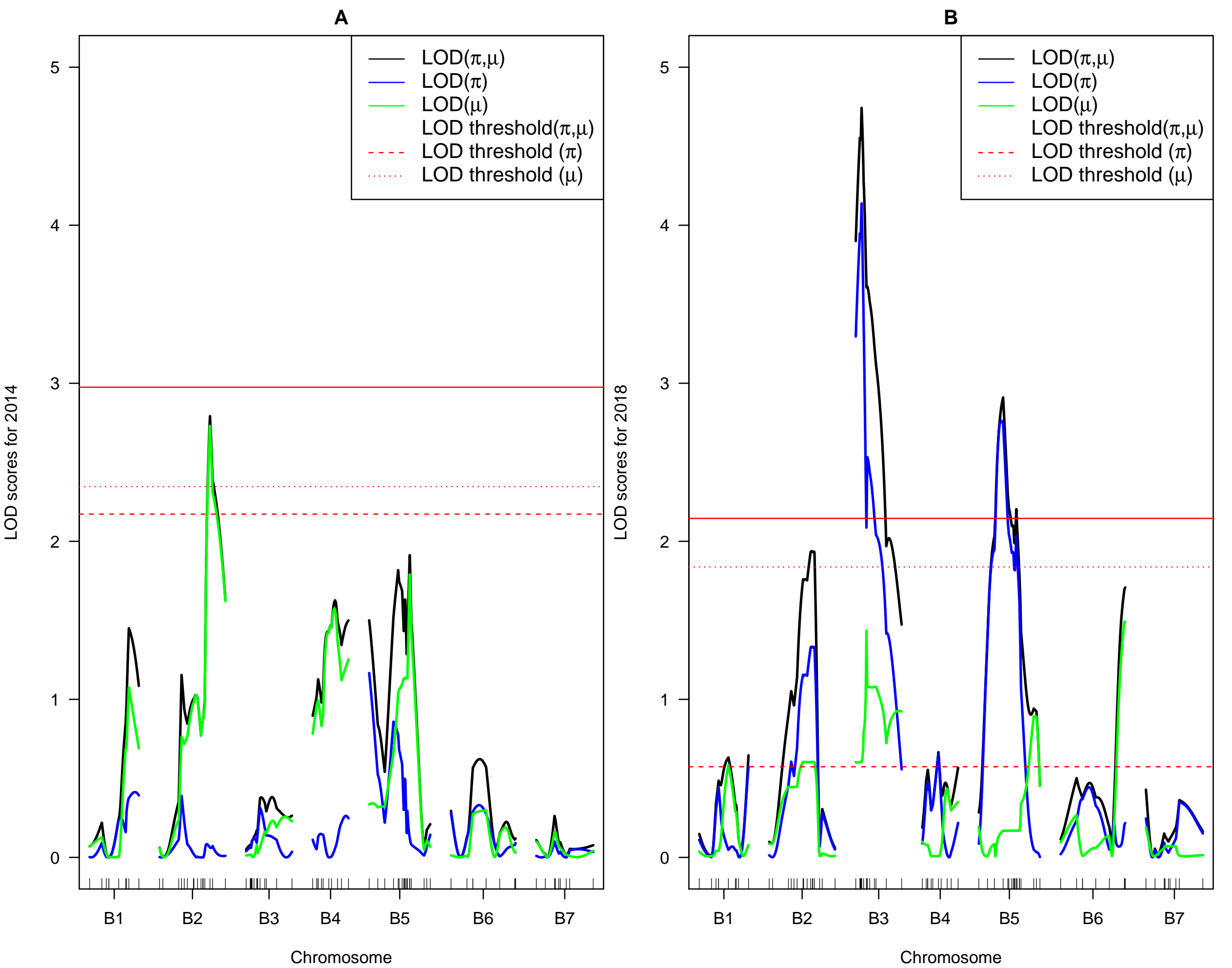


1145 Supp. Fig. 7 QTL mapping associated with black spot disease (BSD) resistance for normally distributed data of HW

1146 population using a normal model with CIM analysis for the male map

1147 Linkage groups are named as follow: "B" for the male map and the number of the linkage group. For HW population,

1148 BSD was scored in three locations: (A) Angers, (B) Bellegarde and (C) Diémoz. LOD score for each year and the

1149 mean of all years were calculated using a Composite Interval Mapping (CIM) method. The same set of colors is used

1150 to represent the different scoring years in the different locations. $\alpha=0.05$ LOD threshold was used for declaring 1151 significant QTL based on 1,000 permutations.

1152

1153 
1154 Supp. Fig. 8 QTL mapping with black spot disease (BSD) resistance using a two-part model approach for non1155 normally distributed data of HW population for the male map

1156 Linkage groups are named as follows: "B" for the male map and the number of the linkage group. A to C: LOD curves

1157 of the two-part model analysis for spike-like distribution of HW BSD scoring years (Angers-2018 (A), Bellegarde-

11582014 (B) and Diémoz-2013 (C)); LOD. $\pi$ (penetrance, equivalent to binary model) displayed in blue, LOD. $\mu$ (severity,

1159 equivalent to normal model for non-spike phenotypes) displayed in green and LOD. $\pi . \mu$ (sum, representing the

1160 complete model) displayed in black; LOD thresholds are displayed in red with $\alpha=0.05$ for declaring significant QTL

1161 based on 1,000 permutations.

1162

1163 
1164 Supp. Fig. 9 QTL mapping with black spot disease resistance using a two-part model approach for non-normally 1165 distributed data of FW and HW populations for the female maps

1166 A: LOD curves for the two-part model for FW population using 2018 scoring year in Angers; B: LOD curves for the 1167 two-part model for HW population using scoring year 2014 in Bellegarde; LOD. $\pi$ (penetrance, equivalent to binary

1168 model) displayed in blue, LOD. $\mu$ (severity, equivalent to normal model for non-spike phenotypes) displayed in green 1169 and LOD. $\pi . \mu$ (sum, representing the complete model) displayed in black; LOD thresholds are displayed in red with $1170 \alpha=0.05$ for declaring significant QTL based on 1,000 permutations.

1171 
1173 Supp. Fig. 10 Co-localization of the Rdrl genes cluster with the QTLs on A1 from OW and FW female maps

1174 Linkage groups (LG) names from female genetic maps are placed above the LG and named A1 - "population name" 1175 and the corresponding chromosome is placed in the middle. Names of markers are on the right and the genetic distances 1176 (in cM) on the left. For the chromosome from the physical map, physical distances are expressed in mega-base (Mb).

1177 TNLs genes and clusters (OB2-A, OB-B, Cluster1 and Cluster2, Menz et al. 2020) are shown in black. The markers 1178 peaks of each QTL are displayed in grey. The active form of $R d r l$ resistance gene ( $m u R d r l A$ ) is located on the cluster2

1179 (Menz et al. 2020). QTLs widest confidence intervals are represented in plain colors on the linkage groups: blue for 1180 the OW female map, yellow for the FW female map and green for the equivalent common widest region on the physical 1181 map.

1182 


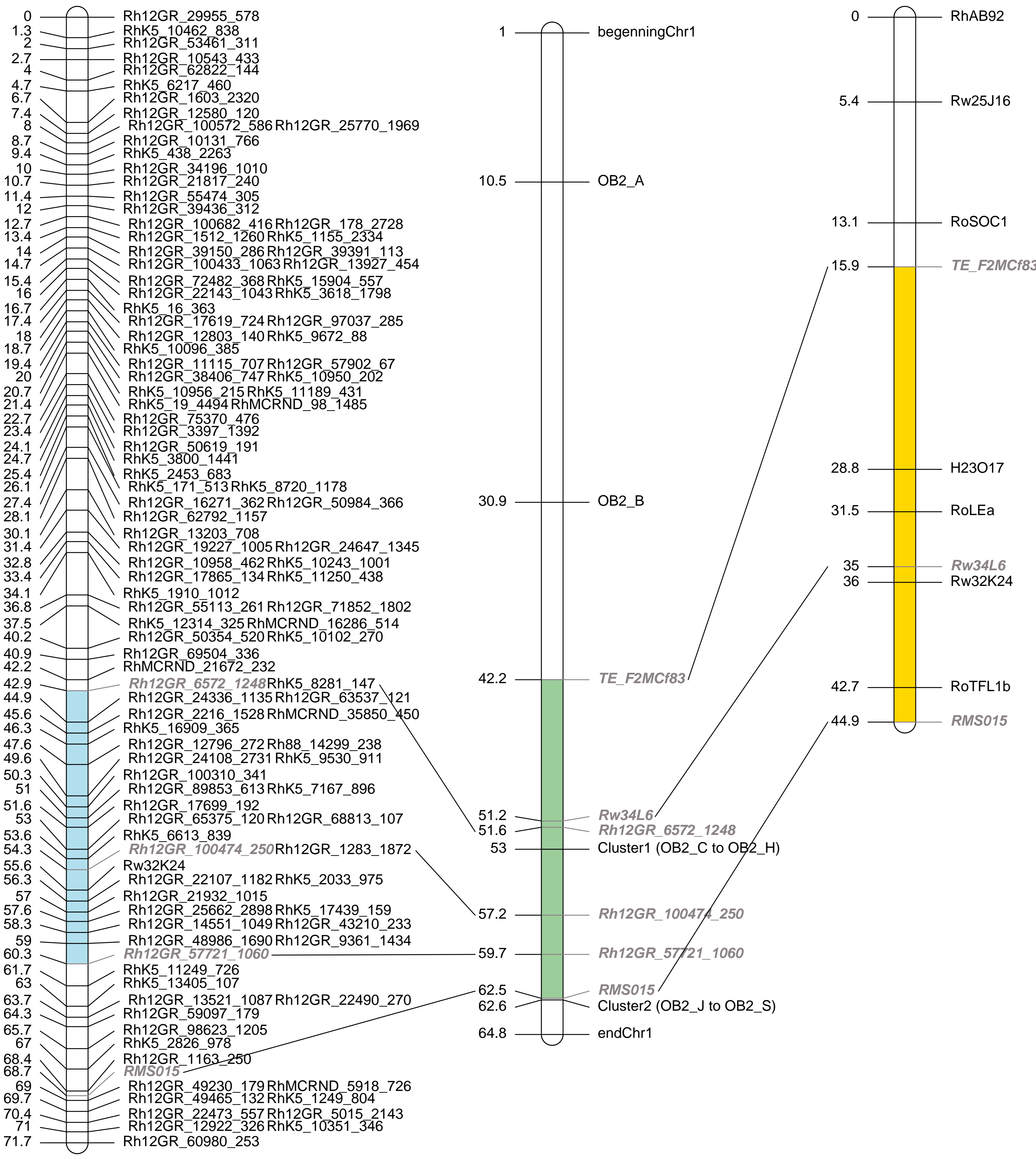




\section{Acknowledgement}

1185 This work was financially supported by RFI Objectif Végétal, the BAP department of the "Institut National 1186 de la Recherche Agronomique et Environnement" (INRAE) and Région Pays de la Loire (support by the CASDAR 1187 project ROGER ${ }^{\circ}$ C-2014-06 from the French Ministry of Agriculture, Agrifood and Forestry). The invaluable 1188 collaboration and work of the ANAN platform (SFR Quasav) for SSR analysis as well as INRA Horticulture 1189 Experimental Facility (Beaucouzé, France), for plant management in experimental fields are gratefully acknowledged.

1190 We thank Martin Leduc for his work on OW maps, Kévin Debray for his work on SNP positioning in the genome and 1191 Briana Gastaldello for the final proofreading of the article.

\section{Material \& correspondence}

1193 Any request for correspondence and materials should be sent to Diana Carolina Lopez Arias (diana.lopez1194 arias@inrae.fr). 